\title{
ON TOTAL SPRINGER REPRESENTATIONS FOR CLASSICAL TYPES
}

\author{
DONGKWAN KIM
}

\begin{abstract}
We give explicit formulas on total Springer representations for classical types. We also describe the characters of restrictions of such representations to a maximal parabolic subgroup isomorphic to a symmetric group. As a result, we give closed formulas for the Euler characteristic of Springer fibers.
\end{abstract}

1. Introduction

2. Definitions and notations

3. Conjugacy classes in Weyl groups

4. Irreducible representations of Weyl groups and the Springer correspondence

5. Main theorems

6. Fibration, stratification and Springer representation

7. Geometric properties for type $B$ and $C$

8. Geometric properties for type $D$

9. Symmetric functions

10. Green and Kostka-Foulkes polynomials

11. Proof of Main Theorem II]

12. Proof of Main Theorem $\square$ for type $B$ and $C$

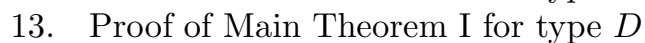

References

\section{INTRODUCTION}

This is a sequel to Kim17. There, we gave inductive formulas to calculate the Euler characteristic of Springer fibers for classical types. Here, we develop its method further and give explicit formulas on total Springer representations for classical types, i.e. the alternating sum of all the cohomology spaces of Springer fibers.

For type $A$, it is well-known that there is a strong connection between Kostka-Foulkes polynomials, Green polynomials, and total Springer representations. More precisely, modified Kostka-Foulkes polynomials (resp. Green polynomials) give the graded multiplicities of irreducible representations in (resp. the graded character values of) total Springer representations. As both polynomials can be computed by the theory of symmetric functions, they provide strong tools to study Springer theory combinatorially. For more information we refer readers to [Lus81, [Mac95, etc.

The graded characters of total Springer representations for other types are also called Green functions, and they play an important role in the representation theory of finite groups of Lie type. It was proved by Kazhdan [Kaz77] (under some restriction on the characteristic of the base field)

Date: May 29, 2018. 
that these functions are identical to the ones defined by Deligne and Lusztig [DL76] using the $\ell$ adic cohomology of Deligne-Lusztig varieties. Later, Lusztig defined generalized Green functions using character sheaves Lus85. His theory provides a way to explicitly calculate Green functions using their orthogonality property, which is now called Lusztig-Shoji algorithm [Sho83, Lus86, Sho88.

Here, we give an alternative way to describe total Springer representations for classical types. The main results of this paper, stated in Section 5 , claim that if we forget the grading and the action of centralizers of nilpotent elements, the total Springer representations for type $B, C$, and $D$ can also be fully described by Kostka-Foulkes polynomials of type $A$. Also, using Green polynomials of type $A$, we give explicit formulas for the restriction of such representations to a maximal parabolic subgroup isomorphic to a symmetric group. This means, roughly speaking, that combinatorics for type $A$ also governs Springer theory for other classical types. This is quite surprising - at least to the author.

As a corollary, we obtain closed formulas for the Euler characteristic of Springer fibers for classical types in terms of Green polynomials. To the best of the author's knowledge, there was no elementary way to calculate such numbers. The most common method is to use Lusztig-Shoji algorithm, but it depends on the theory of character sheaves and orthogonality of Green functionsnone of which are elementary. However, our methods only depend on some geometric properties of Springer fibers and the theory of symmetric functions, both of which are simpler. In addition, the previous paper [Kim17] showed that these numbers can be computed inductively, but it does not give a closed formula except in some cases. On the other hand, Kostka-Foulkes and Green polynomials can be easily obtained using combinatorics.

This paper is organized as follows: in Section 2 we recall the basic definitions and notations that are frequently used in this paper; in Section 3 we describe parametrizations of conjugacy classes in Weyl groups and nilpotent conjugacy classes in Lie algebras for classical types; in Section 4 we give parametrizations of irreducible representations of Weyl groups and their relation to Springer correspondence; in Section 5 we state the main results of this paper; Sections 6 6 are devoted to investigating some geometric properties of Springer fibers and total Springer representations; in Section 9 we recall the theory of symmetric functions and its relation to representation theory; in Section 10 we prove some formulas about Green and Kostka-Foulkes polynomials; and finally, in Sections 11$] 13$ we prove the main theorems.

Acknowledgement. The author wishes to thank George Lusztig for having stimulating discussions with him and checking the draft of this paper. He is grateful to Jim Humphreys for his detailed remarks which help improve the readability of this paper. Also he thanks Gus Lonergan and Toshiaki Shoji for useful comments.

\section{DeFinitions AND nOtations}

2.1. Setup. Let $\mathbb{C}$ be the field of complex numbers and $G$ be a reductive group whose derived group is simple of classical type over $\mathbb{C}$. (Mostly $G$ is one of $G L_{n}(\mathbb{C}), S O_{n}(\mathbb{C})$, or $S p_{n}(\mathbb{C})$ in this paper.) Define $\mathfrak{g}:=\operatorname{Lie} G$ to be the Lie algebra of $G$. We denote the semisimple rank of $G$ by ss-rank $G$. Define $B \subset G$ to be a Borel subgroup of $G$ and $T \subset B$ to be a maximal torus. Also we let $W$ be the Weyl group of $G$, defined by the normalizer of $T$ in $G$ modulo $T$. To avoid ambiguity, we 
sometimes write $W(X)$ for the Weyl group of type $X$. Thus in particular $W\left(A_{n-1}\right)$ is isomorphic to the symmetric group permuting $n$ elements, denoted $\mathfrak{S}_{n}$.

2.2. Partitions. For a partition $\lambda$, we write either $\lambda=\left(\lambda_{1}, \lambda_{2}, \cdots\right)$ for some $\lambda_{1} \geq \lambda_{2} \geq \cdots$ or $\lambda=\left(1^{m_{1}} 2^{m_{2}} \ldots\right)$ to describe its parts. We write $\lambda \vdash n$ if $\sum_{i} \lambda_{i}=n$ or equivalently $\sum_{i} i m_{i}=n$. In this case we also write $|\lambda|=n$. We let $l(\lambda)$ be the length of $\lambda$, i.e. the number of nonzero parts in $\lambda$ or equivalently $\sum_{i} m_{i}$. We denote by $\lambda^{\prime}$ the conjugate of $\lambda$. We often identify partitions with Young diagrams. In this paper we adopt the English notation, thus each part in a partition corresponds to each row in the corresponding Young diagram, and the size of $i$-th row is $\lambda_{i}$.

We say that $\lambda$ is even (resp. odd) if every part in $\lambda$ is even (resp. odd), or equivalently $m_{i}=0$ for $i$ odd (resp. even). In particular, $\lambda$ is called very even if it is even and with even multiplicity, i.e. $m_{i} \in 2 \mathbb{N}$ for any $i$. We say that $\lambda$ is strict if parts of $\lambda$ are pairwise different, that is for $i \neq j$ such that $\lambda_{i}, \lambda_{j} \neq 0$ we have $\lambda_{i} \neq \lambda_{j}$.

For partitions $\lambda=\left(\lambda_{1}, \lambda_{2}, \cdots\right)$ and $\mu=\left(\mu_{1}, \mu_{2}, \cdots\right)$, we define $\lambda \cup \mu$ be the union of two partitions, i.e. the partition of $|\lambda|+|\mu|$ whose parts are $\left\{\lambda_{1}, \lambda_{2}, \cdots\right\} \cup\left\{\mu_{1}, \mu_{2}, \cdots\right\}$ as a multiset. Also for $k \in \mathbb{N}$ we define $k \lambda:=\left(k \lambda_{1}, k \lambda_{2}, \cdots\right)$. For partitions $\lambda$, $\mu$, we write $\lambda \supset \mu$ if $\lambda_{i} \geq \mu_{i}$ for all $i$, i.e. if the Young diagram of $\lambda$ contains that of $\mu$. In this case, we write $\lambda / \mu$ for the corresponding skew partition. Also we write $\lambda \geq \mu$ if $\sum_{i=1}^{r} \lambda_{i} \geq \sum_{i=1}^{r} \mu_{i}$ for any $r \geq 1$.

For $\left\{a_{1}, a_{2}, \cdots\right\} \subset\left\{\lambda_{1}, \lambda_{2}, \cdots\right\}$ as a multiset, we define $\lambda \downarrow_{\left(b_{1}, b_{2}, \cdots\right)}^{\left(a_{1}, \cdots\right)}$ to be the partition obtained by replacing $a_{1}, a_{2}, \cdots$ with $b_{1}, b_{2}, \cdots$ and rearranging rows if necessary. For example, $(6,6,3) \downarrow_{(2)}^{(6)}=(6,3,2)$. Note that $\lambda^{h, i}, \lambda^{v, i}$ in Kim17. can now be written as $\lambda \downarrow_{(i-2)}^{(i)}, \lambda \downarrow_{(i-1, i-1)}^{(i, i)}$, respectively.

Let $b(\lambda):=\sum_{i \geq 1}(i-1) \lambda_{i}=\sum_{j \geq 1}\left(\begin{array}{c}\lambda_{j}^{\prime} \\ 2\end{array}\right)$ be the weighted size of $\lambda$. We also let $z_{\lambda}:=\prod_{i \geq 1} i^{m_{i}} m_{i}$ !, the size of the centralizer of an element of cycle type $\lambda$ in $\mathfrak{S}_{|\lambda|}$. For partitions $\lambda, \mu$ such that $\mu \subset \lambda$, we define ht $(\lambda / \mu)$ to be the height of the skew-partition $\lambda / \mu$, which is given by $\#\left\{i \geq 1 \mid \lambda_{i}>\mu_{i}\right\}-1$. For example, ht $\left(\left(1^{r}\right)\right)=r-1$ for $r \geq 1$.

2.3. 2-core, 2-quotient, and sign of partitions. For a partition $\lambda$, the 2-core of $\lambda$, denoted $\lambda^{(c)}$, is obtained from $\lambda$ by removing as many 2-border strips (i.e. dominos) as possible. Note that $\lambda^{(c)}$ does not depend on the order of removing 2-border strips from $\lambda$. For $|\lambda|$ even (resp. odd), we say the 2-core of $\lambda$ is minimal if $\lambda^{(c)}=\emptyset\left(\right.$ resp. $\lambda^{(c)}=(1)$ ).

In order to define the 2-quotient of $\lambda$, we first choose $m \geq l(\lambda)$ and perform the following.

(1) For $1 \leq i \leq m$, set $a_{i}:=\lambda_{i}+m-i$. In particular, $a_{1}>a_{2}>\cdots>a_{m}$. (Note that $a_{m}$ is zero if $m>l(\lambda)$.)

(2) Separate even and odd integers from $a_{1}, \cdots, a_{m}$, say $2 b_{1}>2 b_{2}>\cdots>2 b_{s}$ and $2 c_{1}+1>$ $2 c_{2}+1>\cdots>2 c_{t}+1$, where $s+t=m$.

(3) Define $\lambda^{(0)}:=\left(b_{1}-s+1, \cdots, b_{s-1}-1, b_{s}\right)$ and $\lambda^{(1)}:=\left(c_{1}-t+1, \cdots, c_{t-1}-1, c_{t}\right)$.

We call $\left(\lambda^{(0)}, \lambda^{(1)}\right)$ the 2 -quotient of $\lambda$. Note that this definition depends on the choice of (the parity of) $m$; indeed, choosing $m \geq l(\lambda)$ of different parity results in swapping $\lambda^{(0)}$ and $\lambda^{(1)}$. In this article we always choose $m$ to be even. Thus for example, if $\lambda=(10)$ then we may choose $m=2$ and get $\left(\lambda^{(0)}, \lambda^{(1)}\right)=(\emptyset,(5))$. 
We define $\varepsilon_{\lambda}:=(-1)^{b(\lambda)}=(-1)^{\sum_{i \geq 1} \lambda_{2 i}}$ to be the sign of $\lambda$. Also for $\mu \subset \lambda$, we let $\epsilon_{2}(\lambda / \mu)$ be the 2-sign of $\lambda / \mu$, which is defined as follows. If it is impossible to fill the Young diagram of $\lambda / \mu$ with dominoes, then set $\epsilon_{2}(\lambda / \mu)=0$. Otherwise, choose any domino filling of $\lambda / \mu$ and set $\epsilon_{2}(\lambda / \mu)$ to be 1 (resp. -1) if the number of vertical dominoes in this filling (with respect to the English notation) is even (resp. odd). Note that this does not depend on the choice of domino fillings. Also for $\mu \subset \lambda$, it is easy to show that

$$
\varepsilon_{\lambda}=\varepsilon_{\mu} \epsilon_{2}(\lambda / \mu), \text { thus in particular, } \varepsilon_{\lambda}=\varepsilon_{\lambda(c)} \epsilon_{2}\left(\lambda / \lambda^{(c)}\right) .
$$

2.4. $\ell$-adic cohomology. For a variety $X$ over $\mathbb{C}$, we define $S h(X)$ to be the category of constructible $\ell$-adic sheaves on $X$. Also define $H^{*}(X):=\bigoplus_{i \in \mathbb{Z}}(-1)^{i} H^{i}(X)$ to be the alternating sum of $\ell$-adic cohomology spaces of $X$ as a virtual vector space. We define $\mathcal{X}(X):=\operatorname{dim} H^{*}(X)=$ $\sum_{i \in \mathbb{Z}}(-1)^{i} \operatorname{dim} H^{i}(X)$ to be the Euler characteristic of $X$. Also we define $H^{\text {top }}(X)$ to be $H^{2 \operatorname{dim} X}(X)$.

2.5. Springer fibers. Let $\mathcal{B}:=G / B$ be the flag variety of $G$, and for $N \in \mathfrak{g}$ define $\mathcal{B}_{N}$ to be the Springer fiber of $N$, i.e.

$$
\mathcal{B}_{N}:=\left\{g B \in \mathcal{B} \mid \operatorname{Ad}(g)^{-1} N \in \operatorname{Lie} B\right\} .
$$

Then the $\ell$-adic cohomology of $\mathcal{B}_{N}$ is naturally equipped with the action of $W$, called the Springer representation, originally defined by [Spr76. Here we adopt the convention of [Lus81, which differs from the former one by tensoring with the sign representation of $W$. We also call $H^{*}\left(\mathcal{B}_{N}\right)$, as a representation of $W$, the total Springer representation corresponding to $N$. As Springer fibers have vanishing odd cohomology dCLP88, in fact it is an actual representation of $W$.

2.6. Characters of a finite group. For a finite group $\mathcal{G}$, we define $i d \in \mathcal{G}$ to be the identity element of $\mathcal{G}$ and also define $\operatorname{Id}_{\mathcal{G}}$ to be the trivial representation of $\mathcal{G}$. Also for a Weyl group $W$, we define $\operatorname{sgn}_{W}$ to be the sign representation of $W$.

Let $\mathcal{R}(\mathcal{G})$ be the ring of $\mathbb{Q}$-valued class functions of $\mathcal{G}$. Also for a $\mathbb{Q}$-algebra $A$, we write $\mathcal{R}(\mathcal{G})_{A}:=$ $\mathcal{R}(\mathcal{G}) \otimes_{\mathbb{Q}} A$. Then there exists a homomorphism from the Grothendieck group of the category of finite dimensional complex representations of $\mathcal{G}$ to $\mathcal{R}(\mathcal{G})_{\mathbb{C}}$ which sends a (virtual) representation of $\mathcal{G}$ to its trace function, i.e. its character. We denote such homomorphism by ch. In other words, for any (virtual) representation $M$ and for any $g \in \mathcal{G}$ we have $\operatorname{ch} M(g)=\operatorname{tr}(g, M)$. If $\mathcal{G}$ is the Weyl group $W$ of some reductive group, then the image of ch actually lands in $\mathcal{R}(W)$ since all the complex irreducible representations of $W$ are defined over $\mathbb{Q}$.

There exists a usual scalar product $\langle\rangle:, \mathcal{R}(\mathcal{G})_{\mathbb{C}} \times \mathcal{R}(\mathcal{G})_{\mathbb{C}} \mapsto \mathbb{C}:(f, g) \mapsto \frac{1}{\# \mathcal{G}} \sum_{x \in \mathcal{G}} f(x) \overline{g(x)}$. For any virtual representations $M, N$ of $\mathcal{G}$, we also define $\langle M, N\rangle:=\langle\operatorname{ch} M, \operatorname{ch} N\rangle$. For a subgroup $\mathcal{H} \subset \mathcal{G}$, we define $\operatorname{Res}_{\mathcal{H}}^{\mathcal{G}}$ and $\operatorname{Ind}_{\mathcal{H}}^{\mathcal{G}}$ to be the usual restriction and induction functor. They are both well-defined on finite dimensional complex representations and class functions. Also, they are adjoint to each other with respect to the scalar product defined above.

For $g \in \mathcal{G}$ which commutes with all elements in $\mathcal{H}$, we define $\operatorname{Res}_{g \cdot \mathcal{H}}^{\mathcal{G}}$ to be the restriction of a class function of $\mathcal{G}$ to $g \mathcal{H}=\mathcal{H} g$ (the coset of $\mathcal{H}$ ), considered as a class function of $\mathcal{H}$ under the identification $\mathcal{H} \stackrel{\simeq}{\rightrightarrows} g \mathcal{H}: h \mapsto g h$. (Thus here it is necessary to specify $g$ in the notation.) Note that even when we choose $M$ to be an actual representation of $\mathcal{G}$, $\operatorname{Res}_{g \cdot \mathcal{H}}^{\mathcal{G}} \operatorname{ch} M$ need not be a character of an actual one of $\mathcal{H}$, e.g. $\operatorname{Res}_{-1 \cdot\{i d\}}^{\mathbb{Z} / 2} \operatorname{chsgn}_{\mathbb{Z} / 2}$ is a virtual representation of the trivial group. 
2.7. Miscellaneous. For a vector space $V$ and a subset $X \subset V$, we define $\operatorname{sp}\{X\}$ to be the span of $X$ in $V$.

\section{Conjugacy Classes in Weyl groups}

Here we recall the parametrizations of conjugacy classes in Weyl groups and nilpotent conjugacy classes in Lie algebras for classical types.

3.1. Type $A$. Let $G=G L_{n}(\mathbb{C})$. Then its Weyl group is isomorphic to $\mathfrak{S}_{n}$. Its conjugacy classes are parametrized by partitions of $n$; to each conjugacy class we associate its cycle type. For $\rho \vdash n$, we write $w_{\rho} \in W$ to be the element of cycle type $\rho$, which is well-defined up to conjugation.

Also, there is a natural bijection between the set of nilpotent conjugacy classes in $\mathfrak{g}$ and the set of partitions of $n$; to each conjugacy class we associate its Jordan type. For $\lambda \vdash n$, we write $N_{\lambda} \in \mathfrak{g}$ to be the nilpotent element of Jordan type $\lambda$, which is again well-defined up to conjugation. For $N=N_{\lambda}$, we define $\operatorname{TSp}(\lambda):=H^{*}\left(\mathcal{B}_{N}\right)$ as a $W$-module.

3.2. Type $B$ and $C$. Let $G=S O_{2 n+1}(\mathbb{C})$ or $G=S p_{2 n}(\mathbb{C})$. Then its Weyl group is isomorphic to $\mathfrak{S}_{n} \ltimes(\mathbb{Z} / 2)^{n}$. Thus its conjugacy classes are parametrized by the set of ordered pairs of partitions $(\rho, \sigma)$ such that $|\rho|+|\sigma|=n$, where $\rho$ and $\sigma$ correspond to $1,-1 \in \mathbb{Z} / 2$, respectively. (ref. Mac95, pp.170-171, 178]) For such pair $(\rho, \sigma)$, we write $w_{(\rho, \sigma)} \in W$ to be the element which is contained in the conjugacy class parametrized by $(\rho, \sigma)$. It is clearly well-defined up to conjugation.

If $G$ is of type $B$, by taking the Jordan type we have a bijection between nilpotent conjugacy classes in $\mathfrak{g}$ and the set of partitions $\lambda \vdash 2 n+1$ each of whose even parts has even multiplicity. Similarly, if $G$ is of type $C$, by taking the Jordan type we have a bijection between nilpotent conjugacy classes in $\mathfrak{g}$ and the set of partitions $\lambda \vdash 2 n$ each of whose odd parts has even multiplicity. We similarly write $N_{\lambda} \in \mathfrak{g}$ for such $\lambda$ to denote the nilpotent element of Jordan type $\lambda$, which is well-defined up to conjugation. We define $\operatorname{TSp}(\lambda)$ similarly to type $A$.

3.3. Type $D$. Let $G=S O_{2 n}(\mathbb{C})$. Then its Weyl group can be regarded as a subgroup of $\mathfrak{S}_{n} \ltimes(\mathbb{Z} / 2)^{n}$ of order 2. Then a conjugacy class of $\mathfrak{S}_{n} \ltimes(\mathbb{Z} / 2)^{n}$ parametrized by $(\rho, \sigma)$ has a nontrivial intersection with $W$ if and only if $l(\sigma)$ is even. If so, the intersection is again a conjugacy class in $W$ except when $\sigma=\emptyset$ and $\rho$ is even, in which case it splits into two conjugacy classes. Thus there exists a parametrization of the conjugacy classes of $W$ by the set

$$
\{(\rho, \sigma)|| \rho|+| \sigma \mid=n, l(\sigma) \in 2 \mathbb{N}, \rho \text { is not even if } \sigma=\emptyset\} \cup\{(\rho, \emptyset) \pm \mid \rho \vdash n, \rho \text { is even })\} .
$$

Here $(\rho, \emptyset)+$ and $(\rho, \emptyset)$ - correspond to two conjugacy classes in $W$ contained in the conjugacy class of $\mathfrak{S}_{n} \ltimes(\mathbb{Z} / 2)^{n}$ parametrized by $(\rho, \emptyset)$. We write $w_{(\rho, \sigma)}, w_{(\rho, \emptyset) \pm}$ to denote the corresponding elements in $W$ which are well-defined up to conjugation.

For a partition $\lambda \vdash 2 n$ each of whose even parts has even multiplicity, there exists a unique nilpotent conjugacy class in $\mathfrak{g}$ whose Jordan type is $\lambda$ unless $\lambda$ is very even; if so, then there exist two such classes. Thus the set of nilpotent conjugacy classes in $\mathfrak{g}$ is parametrized by

$$
\left\{\lambda=\left(1^{m_{1}} 2^{m_{2}} \ldots\right) \vdash 2 n \mid m_{2 i} \in 2 \mathbb{N} \text { for } i \in \mathbb{Z}_{\geq 1}, \lambda \text { is not very even }\right\} \cup\{\lambda \pm \mid \lambda \vdash 2 n \text { is very even }\} .
$$

We write $N_{\lambda}, N_{\lambda \pm}$ to denote the corresponding nilpotent elements in $\mathfrak{g}$ which are well-defined up to conjugation. If $N=N_{\lambda}$ for $\lambda$ not very even, we define $\mathbf{T S p}(\lambda)$ similarly to type $A$ and also put 
$\widetilde{\mathbf{T S p}}(\lambda):=\mathbf{T S p}(\lambda) \oplus \mathbf{T S p}(\lambda)$. If $\lambda$ is very even, we define $\mathbf{T S p}(\lambda \pm)=H^{*}\left(\mathcal{B}_{N}\right)$ for $N=N_{\lambda \pm}$ and put $\widetilde{\mathbf{T S p}}(\lambda):=\mathbf{T S p}(\lambda+) \oplus \mathbf{T S p}(\lambda-)$.

In order to remove ambiguity from the choice of the sign \pm , we proceed as follows. We construct embeddings

$$
\mathrm{SO}_{4}(\mathbb{C}) \hookrightarrow \mathrm{SO}_{6}(\mathbb{C}) \hookrightarrow S \mathrm{O}_{8}(\mathbb{C}) \hookrightarrow \cdots \hookrightarrow S \mathrm{O}_{2 n}(\mathbb{C}) \hookrightarrow S \mathrm{O}_{2 n+2}(\mathbb{C}) \hookrightarrow \cdots
$$

which induces embeddings of Weyl groups

$$
W\left(D_{2}\right) \hookrightarrow W\left(D_{3}\right) \hookrightarrow W\left(D_{4}\right) \hookrightarrow \cdots \hookrightarrow W\left(D_{n}\right) \hookrightarrow W\left(D_{n+1}\right) \hookrightarrow \cdots
$$

such that for any $i<j$, the image of $W\left(D_{i}\right) \hookrightarrow W\left(D_{j}\right)$ is a parabolic subgroup of $W\left(D_{j}\right)$. Thus the set of simple reflections of $W\left(D_{i}\right)$ can be regarded as a subset of that of $W\left(D_{j}\right)$ for $i<j$. Here we also require that the simple reflections of $W\left(D_{2}\right)$ correspond to two extremal points in the Dynkin diagram of $D_{n}$ for any $n \geq 2$.

There exists an involution $\tau_{n}$ on $S O_{2 n}(\mathbb{C})$ which gives the nontrivial involution on the Dynkin diagram and also on $W\left(D_{n}\right)$. We may choose $\tau_{n}$ for each $n \geq 2$ such that for any $m<n$ we have $\left.\tau_{n}\right|_{S O_{2 m}(\mathbb{C})}=\tau_{m}$ under the embeddings above. Thus we drop the subscript and simply write $\tau$ to be this nontrivial involution on any $S O_{2 n}(\mathbb{C})$. (Even when $n=4$, there is no ambiguity since the embedding $\mathrm{SO}_{8}(\mathbb{C}) \hookrightarrow S O_{10}(\mathbb{C})$ completely determines $\tau_{4}$.)

Now once and for all, we fix the labeling $s_{+}, s_{-}$of two simple reflections in $W\left(D_{2}\right) \simeq \mathbb{Z} / 2 \times \mathbb{Z} / 2$, and regard them as simple reflections in $W\left(D_{n}\right)$ for any $n \in \mathbb{Z}_{>2}$. We write $\mathfrak{S}_{n+}$ (resp. $\mathfrak{S}_{n-}$ ) to be the parabolic subgroup of $W$ generated by all the simple reflections but $s_{-}$(resp. $\left.s_{+}\right)$. Similarly, we write $L_{+}$(resp. $L_{-}$) to be the Levi subgroup (which contains $T$ ) of a parabolic subgroup (which contains $B$ ) whose Weyl group is $\mathfrak{S}_{n+}\left(\right.$ resp. $\left.\mathfrak{S}_{n-}\right)$.

For a conjugacy class which corresponds to either $(\rho, \emptyset)+$ or $(\rho, \emptyset)$ - for some $\rho$ even, we say that it is parametrized by $(\rho, \emptyset)+(\operatorname{resp} .(\rho, \emptyset)-)$ if its intersection with $\mathfrak{S}_{n+}$ (resp. $\left.\mathfrak{S}_{n-}\right)$ is nonempty. Also, for a nilpotent conjugacy class whose Jordan type is $\lambda \vdash 2 n$ very even, we say that it is parametrized by $\lambda+$ (resp. $\lambda_{-}$) if its intersection with the Lie algebra of $L_{+}$(resp. $L_{-}$) is nonempty. Then one can show that these notions are indeed well-defined. Also note that $\tau$ swaps + and - in both parametrizations.

These parametrizations have a following advantage. For $\lambda$ very even, by [Lus04] $\mathbf{T S p}(\lambda+)$ (resp. $\mathbf{T S p}(\lambda-))$ is an induced character from a parabolic subgroup contained in $\mathfrak{S}_{n+}\left(\right.$ resp. $\left.\mathfrak{S}_{n-}\right)$. As conjugacy classes in $W$ parametrized by $(\rho, \emptyset)+($ resp. $(\rho, \emptyset)-)$ does not intersect with $\mathfrak{S}_{n-}$ (resp. $\left.\mathfrak{S}_{n+}\right)$, the following observation is a direct consequence of Mackey's formula.

Lemma 3.1. Let $G=S O_{2 n}(\mathbb{C})$. For $\rho \vdash n$ even and $\lambda \vdash 2 n$ very even, we have

$$
\operatorname{ch} \mathbf{T S p}\left(\lambda_{-}\right)\left(w_{(\rho, \emptyset)+}\right)=\operatorname{ch} \mathbf{T S p}(\lambda+)\left(w_{(\rho, \emptyset)-}\right)=0 .
$$

\section{Irreducible Representations of Weyl groups and the Springer correspondence}

Here we recall the parametrizations of irreducible representations of Weyl groups for classical types and how they are related to nilpotent orbits in $\mathfrak{g}$ under the Springer correspondence. One may refer to Sho79, Lus79, Car93 for more information. 
4.1. Type $A$. Let $W=\mathfrak{S}_{n}$ be the Weyl group of type $A_{n-1}$. The irreducible representations of $W$ (up to isomorphism) are parametrized by partitions of $n$. For $\lambda \vdash n$, we write $\chi^{\lambda}$ to denote such representation. Thus for example $\chi^{(n)}=\operatorname{Id}_{W}$ and $\chi^{\left(1^{n}\right)}=\operatorname{sgn}_{\mathfrak{S}_{n}}$. Then the Springer correspondence is simply given by $H^{\text {top }}\left(\mathcal{B}_{N}\right) \simeq \chi^{\lambda}$ for $N=N_{\lambda}$.

4.2. Type $B$ and $C$. Let $W=\mathfrak{S}_{n} \ltimes(\mathbb{Z} / 2)^{n}$ be the Weyl group of type $B C_{n}$. The irreducible representations of $W$ (up to isomorphism) are parametrized by ordered pairs of partitions $(\lambda, \mu)$ such that $|\lambda|+|\mu|=n$. For such $(\lambda, \mu)$, we write $\chi^{(\lambda, \mu)}$ to denote such representation. Thus for example $\chi^{((n), \emptyset)}=\operatorname{Id}_{W}$ and $\chi^{\left(\emptyset,\left(1^{n}\right)\right)}=\operatorname{sgn}_{W}$.

If $G$ is of type $B$, then for a partition $\lambda \vdash 2 n+1$ with minimal 2-core, we define $\chi^{\lambda}:=\chi^{\left(\lambda^{(0)}, \lambda^{(1)}\right)}$. If $G$ is of type $C$, then for a partition $\lambda \vdash 2 n$ with minimal 2-core, we also define $\chi^{\lambda}:=\chi^{\left(\lambda^{(1)}, \lambda^{(0)}\right)}$. (Note the difference from type $B$.) If its 2 -core is not minimal, then we simply put $\chi^{\lambda}=0$. Then the Springer correspondence is given by $H^{t o p}\left(\mathcal{B}_{N}\right)^{Z_{G}(N)} \simeq \chi^{\lambda}$ for $N=N_{\lambda}$. Here, $Z_{G}(N)$ is the stabilizer of $N$ in $G$.

4.3. Type $D$. Let $W$ be the Weyl group of type $D_{n}$. The irreducible representations of $W$ (up to isomorphism) are parametrized by unordered pairs of partitions $\{\lambda, \mu\}$ such that $|\lambda|+|\mu|=n$ except when $\lambda=\mu$, in which case there are two irreducible representations corresponding to $\{\lambda, \lambda\}$. For such $(\lambda, \mu)$ with $\lambda \neq \mu$, we write $\chi^{\{\lambda, \mu\}}$ to denote such representation. Thus for example $\chi^{\{(n), \emptyset\}}=\operatorname{Id}_{W}$ and $\chi^{\left\{\emptyset,\left(1^{n}\right)\right\}}=\chi^{\left\{\left(1^{n}\right), \emptyset\right\}}=\operatorname{sgn}_{W}$.

In the case when $\lambda=\mu$, we write $\chi^{\{\lambda, \lambda\}+}, \chi^{\{\lambda, \lambda\}-}$ to denote such two representations corresponding to $\{\lambda, \lambda\}$. The choice of the sign is characterized by the property that $\left\langle\operatorname{Ind}_{\mathfrak{S}_{n \pm}}^{W} \operatorname{Id}_{\mathfrak{S}_{n \pm}}, \chi^{\{\lambda, \lambda\} \mp}\right\rangle=$ 0 . Here $\mathfrak{S}_{n+}\left(\right.$ resp. $\left.\mathfrak{S}_{n-}\right)$ is the parabolic subgroup of $W$ generated by all the simple reflections but $s_{-}\left(\right.$resp. $\left.s_{+}\right)$, see Section 3.3

For a partition $\lambda \vdash 2 n$ with minimal 2-core, if $\lambda$ is not very even then $\lambda^{(0)} \neq \lambda^{(1)}$ and we define $\chi^{\lambda}:=\chi^{\left\{\lambda^{(0)}, \lambda^{(1)}\right\}}$. If $\lambda$ is very even, then $\lambda^{(0)}=\lambda^{(1)}$ and we define $\chi^{\lambda \pm}:=\chi^{\left\{\lambda^{(0)}, \lambda^{(0)}\right\} \pm}$. Then the Springer correspondence is described as follows. For $\lambda$ not very even, we have $H^{\text {top }}\left(\mathcal{B}_{N}\right)^{Z_{G}(N)} \simeq \chi^{\lambda}$ for $N=N_{\lambda}$. Here, again $Z_{G}(N)$ is the stabilizer of $N$ in $G$. If $\lambda$ is very even, then we have $H^{t o p}\left(\mathcal{B}_{N}\right) \simeq \chi^{\lambda \pm}$ for $N=N_{\lambda \pm}$. (In this case $Z_{G}(N)$ is connected and thus $H^{\text {top }}\left(\mathcal{B}_{N}\right)^{Z_{G}(N)}=$ $H^{\text {top }}\left(\mathcal{B}_{N}\right)$.)

\section{MAIN THEOREMS}

We are ready to state the main results of this papar.

Main Theorem I. For partitions $\lambda$ and $\mu$ such that $|\lambda|=|\mu|$, let $K_{\mu, \lambda}(t)$ be the Kostka-Foulkes polynomial, ref. [Mac95, Chapter III.6].

(1) Let $G=S O_{2 n+1}(\mathbb{C})$ or $G=S p_{2 n}(\mathbb{C})$ and $\lambda$ be a Jordan type of some nilpotent element in g. Then

$$
\operatorname{ch} \mathbf{T S p}(\lambda)=\sum_{\mu \vdash|\lambda|} \varepsilon_{\lambda} \varepsilon_{\mu} K_{\mu, \lambda}(-1) \operatorname{ch} \chi^{\mu} .
$$


(2) Let $G=S O_{2 n}(\mathbb{C})$ and $\lambda \vdash 2 n$ be a Jordan type of some nilpotent element in $\mathfrak{g}$ whicn is not very even. Then (note that $\varepsilon_{\mu}=1$ if $\mu$ is very even)

$$
\operatorname{ch} \mathbf{T S p}(\lambda)=\frac{1}{2} \sum_{\substack{\mu \vdash 2 n \\ \text { not very even }}} \varepsilon_{\lambda} \varepsilon_{\mu} K_{\mu, \lambda}(-1) \operatorname{ch} \chi^{\mu}+\frac{1}{2} \sum_{\substack{\mu \vdash 2 n \\ \text { very even }}} \varepsilon_{\lambda} K_{\mu, \lambda}(-1) \operatorname{ch}\left(\chi^{\mu+} \oplus \chi^{\mu-}\right) .
$$

If $\lambda$ is very even, then set $\tilde{\lambda} \vdash n / 2$ to be such that $\lambda=2 \tilde{\lambda} \cup 2 \tilde{\lambda}$. Define $\tilde{\mu} \vdash n / 2$ similarly if $\mu$ is very even. Then, (note that $\varepsilon_{\lambda}=1$, and also $\varepsilon_{\mu}=1$ if $\mu$ is very even)

$$
\begin{aligned}
\operatorname{ch} \mathbf{T S p}(\lambda+)= & \frac{1}{2} \sum_{\substack{\mu \vdash 2 n \\
\text { not very even }}} \varepsilon_{\mu} K_{\mu, \lambda}(-1) \operatorname{ch} \chi^{\mu} \\
& +\sum_{\substack{\mu \vdash 2 n \\
\text { very even }}}\left(\frac{K_{\mu, \lambda}(-1)+K_{\tilde{\mu}, \tilde{\lambda}}}{2} \operatorname{ch} \chi^{\mu+}+\frac{K_{\mu, \lambda}(-1)-K_{\tilde{\mu}, \tilde{\lambda}}}{2} \operatorname{ch} \chi^{\mu-}\right), \\
\operatorname{ch~} \mathbf{T S p}(\lambda-)= & \frac{1}{2} \sum_{\substack{\mu \vdash 2 n \\
\text { not very even }}} \varepsilon_{\mu} K_{\mu, \lambda}(-1) \operatorname{ch} \chi^{\mu} \\
& +\sum_{\substack{\mu \vdash 2 n \\
\text { very even }}}\left(\frac{K_{\mu, \lambda}(-1)-K_{\tilde{\mu}, \tilde{\lambda}}}{2} \operatorname{ch} \chi^{\mu+}+\frac{K_{\mu, \lambda}(-1)+K_{\tilde{\mu}, \tilde{\lambda}}}{2} \operatorname{ch} \chi^{\mu-}\right) .
\end{aligned}
$$

Here $K_{\tilde{\mu}, \tilde{\lambda}}=K_{\tilde{\mu}, \tilde{\lambda}}(1)$ is the Kostka number.

Remark. For $G=G L_{n}(\mathbb{C})$ there is a graded analogue

$$
\sum_{i \in \mathbb{Z}}(-1)^{i} t^{i / 2} \operatorname{ch~} H^{i}\left(\mathcal{B}_{N_{\lambda}}\right)=\sum_{\mu \vdash n} t^{b(\lambda)} K_{\mu, \lambda}\left(t^{-1}\right) \operatorname{ch} \chi^{\mu} .
$$

Remark. One needs to be careful when trying to calculate $\left\langle\mathbf{T S p}(\lambda), \chi^{\mu}\right\rangle$ for type $D$. If $\mu$ is not very even, then there exists $\nu \vdash|\lambda|$ such that $\mu \neq \nu, \mu^{(0)}=\nu^{(1)}$, and $\mu^{(1)}=\nu^{(0)}$. Thus we have

$$
\left\langle\mathbf{T S p}(\lambda), \chi^{\mu}\right\rangle=\left\langle\mathbf{T S p}(\lambda), \chi^{\nu}\right\rangle=\frac{1}{2} \varepsilon_{\lambda}\left(\varepsilon_{\mu} K_{\mu, \lambda}(-1)+\varepsilon_{\nu} K_{\nu, \lambda}(-1)\right) .
$$

Indeed, the author conjectures that $\varepsilon_{\mu} K_{\mu, \lambda}(-1)=\varepsilon_{\nu} K_{\nu, \lambda}(-1)$. Thus it is likely that

$$
\left\langle\mathbf{T S p}(\lambda), \chi^{\mu}\right\rangle=\left\langle\mathbf{T S p}(\lambda), \chi^{\nu}\right\rangle=\varepsilon_{\lambda} \varepsilon_{\mu} K_{\mu, \lambda}(-1)=\varepsilon_{\lambda} \varepsilon_{\nu} K_{\nu, \lambda}(-1) .
$$

The following theorem may be regarded as a corollary of Main Theorem [

Main Theorem II. For partitions $\lambda$ and $\rho$ such that $|\lambda|=|\rho|$, let $\mathcal{Q}_{\rho}^{\lambda}(t)$ be the Green polynomial defined in Gre55].

(1) Let $G=S O_{2 n+1}(\mathbb{C})$ and $\lambda \vdash 2 n+1$ be a Jordan type of some nilpotent element in $\mathfrak{g}$. Then

$$
\operatorname{ch} \mathbf{T S p}(\lambda)\left(w_{(\rho, \emptyset)}\right)=\mathcal{Q}_{2 \rho \cup(1)}^{\lambda}(-1), \quad \text { thus } \quad \mathcal{X}\left(\mathcal{B}_{N_{\lambda}}\right)=\mathcal{Q}_{\left(1^{1} 2^{n}\right)}^{\lambda}(-1) .
$$

(2) Let $G=S p_{2 n}(\mathbb{C})$ and $\lambda \vdash 2 n$ be a Jordan type of some nilpotent element in $\mathfrak{g}$. Then

$$
\operatorname{ch} \mathbf{T S p}(\lambda)\left(w_{(\rho, \emptyset)}\right)=\mathcal{Q}_{2 \rho}^{\lambda}(-1), \quad \text { thus } \quad \mathcal{X}\left(\mathcal{B}_{N_{\lambda}}\right)=\mathcal{Q}_{\left(2^{n}\right)}^{\lambda}(-1) .
$$


(3) Let $G=S O_{2 n}(\mathbb{C})$ and $\lambda \vdash 2 n$ be a Jordan type of some nilpotent element in $\mathfrak{g}$. If $\lambda$ is not very even and $\rho$ is not even, then

$$
\operatorname{ch} \mathbf{T S p}(\lambda)\left(w_{(\rho, \emptyset)}\right)=\frac{1}{2} \mathcal{Q}_{2 \rho}^{\lambda}(-1), \quad \text { thus } \quad \mathcal{X}\left(\mathcal{B}_{N_{\lambda}}\right)=\frac{1}{2} \mathcal{Q}_{\left(2^{n}\right)}^{\lambda}(-1) .
$$

If $\lambda$ is not very even and $\rho$ is even, then

$$
\operatorname{ch} \mathbf{T S p}(\lambda)\left(w_{(\rho, \emptyset) \pm}\right)=\frac{1}{2} \mathcal{Q}_{2 \rho}^{\lambda}(-1) .
$$

If $\lambda$ is very even and $\rho \vdash n$ is not even, then

$$
\operatorname{ch} \operatorname{TSp}(\lambda \pm)\left(w_{(\rho, \emptyset)}\right)=\frac{1}{2} \mathcal{Q}_{2 \rho}^{\lambda}(-1), \quad \text { thus } \quad \mathcal{X}\left(\mathcal{B}_{N_{\lambda \pm}}\right)=\frac{1}{2} \mathcal{Q}_{\left(2^{n}\right)}^{\lambda}(-1) .
$$

If $\lambda$ is very even and $\rho$ is even, then

$$
\operatorname{ch} \mathbf{T S p}(\lambda \pm)\left(w_{(\rho, \emptyset) \pm}\right)=\mathcal{Q}_{2 \rho}^{\lambda}(-1), \quad \text { and } \quad \operatorname{ch} \mathbf{T S p}(\lambda \mp)\left(w_{(\rho, \emptyset) \pm}\right)=0 .
$$

Remark. There is also a graded analogue for $G=G L_{n}(\mathbb{C})$

$$
\sum_{i \in \mathbb{Z}}(-1)^{i} t^{i / 2} \operatorname{ch} H^{i}\left(\mathcal{B}_{N_{\lambda}}\right)\left(w_{\rho}\right)=\mathcal{Q}_{\rho}^{\lambda}(t), \quad \text { thus } \quad \chi\left(\mathcal{B}_{N_{\lambda}}\right)=\mathcal{Q}_{\left(1^{n}\right)}^{\lambda}(1)
$$

that was first proved in Spr71].

\section{Fibration, stratification and Springer Representation}

We first recall [HS79, Theorem 5.1]. For a parabolic subgroup $P \supset B$ of $G$, let $P=L \cdot U_{P}$ be its Levi decomposition such that $T \subset L$. We also let $\mathcal{P}:=G / P$ be the partial flag variety of $G$ corresponding to $P$. For a nilpotent element $N \in \mathfrak{g}$, we define

$$
\mathcal{P}_{N}:=\left\{g P \in \mathcal{P} \mid \operatorname{Ad}(g)^{-1} N \in \operatorname{Lie} P\right\} .
$$

Then there exists a natural morphism $\pi_{P}: \mathcal{B}_{N} \rightarrow \mathcal{P}_{N}: g B \mapsto g P$. We define $W_{P} \subset W$ to be the Weyl group of $P$ considered as a parabolic subgroup of $W$.

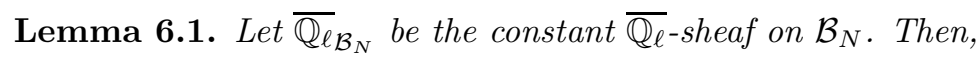

(1) $R^{j} \pi_{!}\left(\overline{\mathbb{Q}}_{\mathcal{B}_{N}}\right) \in \operatorname{Sh}\left(\mathcal{P}_{N}\right)$ for $j \in \mathbb{Z}$ has a structure of sheaves of $W_{P}$-modules.

(2) Let $M:=\left.\operatorname{Ad}(g)^{-1} N\right|_{\text {Lie } L}$ be the image of $\operatorname{Ad}(g)^{-1} N \subset \operatorname{Lie} P$ under the projection Lie $P \rightarrow$ Lie $P /$ Lie $U_{P} \simeq$ Lie $L$. Then for any $g P \in \mathcal{P}_{N},\left.R^{j} \pi_{!}\left(\overline{\mathbb{Q}}_{\mathcal{B}_{N}}\right)\right|_{g P} \simeq H^{j}\left(\mathcal{B}(L)_{M}\right)$ as $W_{P^{-}}$ modules. Here, $\mathcal{B}(L):=L / L \cap B$ is the flag variety of $L$, and the $W_{P}$-module structure on $H^{j}\left(\mathcal{B}(L)_{M}\right)$ is defined as the Springer representation associated to $L$.

(3) $H^{i}\left(\mathcal{P}_{N}, R^{j} \pi !\left(\overline{\mathbb{Q}}_{\mathcal{B}_{N}}\right)\right) \Rightarrow H^{i+j}\left(\mathcal{B}_{N}\right)$ is a spectral sequence of $W_{P}$-modules where the $W_{P^{-}}$ module structure on $H^{i+j}\left(\mathcal{B}_{N}\right)$ is given by the restriction of the Springer representation of $W$ to $W_{P}$.

Suppose $P \subset G$ is a parabolic subgroup of $G$ such that $W_{P} \simeq \mathfrak{S}_{k} \times W^{\prime}$ for some $1 \leq k \leq$ ss-rank $G$ where $W^{\prime}$ is a parabolic subgroup of the same type as $W$. Also suppose that there exists a stratification $\mathcal{P}_{N}=\sqcup_{\alpha} X_{\alpha}$ such that on each $X_{\alpha}, H^{*}\left(\pi_{P}^{-1}(x)\right)$ at any $x \in X_{\alpha}$ is isomorphic as $W_{P}$-modules. Then by Lemma 6.1, for any $w \in W_{P}$ and any $x_{\alpha} \in X_{\alpha}$ for each $\alpha$, we have

$$
\operatorname{ch} H^{*}\left(\mathcal{B}_{N}\right)(w)=\sum_{\alpha} \mathcal{X}\left(X_{\alpha}\right) \operatorname{ch} H^{*}\left(\pi_{P}^{-1}\left(x_{\alpha}\right)\right)(w) .
$$


We may find two reductive groups $\tilde{L}, L^{\prime}$ where $\tilde{L}$ is of type $A_{k-1}$ and $L^{\prime}$ is of the same type as $G$, such that Lie $L=\operatorname{Lie} \tilde{L} \oplus \operatorname{Lie} L^{\prime}$. We define $\mathcal{B}(L), \mathcal{B}(\tilde{L}), \mathcal{B}\left(L^{\prime}\right)$ to be the flag varieties of $L, \tilde{L}, L^{\prime}$, respectively. Then $\mathcal{B}(L)=\mathcal{B}(\tilde{L}) \times \mathcal{B}\left(L^{\prime}\right)$. Also we let $\tilde{N}:=\left.\operatorname{Ad}(g)^{-1} N\right|_{\text {Lie } \tilde{L}}, N^{\prime}:=\left.\operatorname{Ad}(g)^{-1} N\right|_{\operatorname{Lie} L^{\prime}}$. For $w \in W_{P}$, we write $w=c w^{\prime}$ for some $c \in \mathfrak{S}_{k}$ and $w^{\prime} \in W^{\prime}$. Then for any $g P \in \mathcal{P}_{N}$ we have

therefore

$$
\pi_{P}^{-1}(g P) \simeq \mathcal{B}(\tilde{L})_{\tilde{N}} \times \mathcal{B}\left(L^{\prime}\right)_{N^{\prime}}
$$

$$
\operatorname{ch} H^{*}\left(\pi^{-1}(g P)\right)(w)=\operatorname{ch} H^{*}\left(\mathcal{B}(\tilde{L})_{\tilde{N}}\right)(c) \cdot \operatorname{ch} H^{*}\left(\mathcal{B}\left(L^{\prime}\right)_{N^{\prime}}\right)\left(w^{\prime}\right)
$$

We further assume that $c \in \mathfrak{S}_{k}$ is a Coxeter element, i.e. a $k$-cycle. If $\tilde{N}$ is not regular, then $\operatorname{ch} H^{*}\left(\mathcal{B}(\tilde{L})_{\tilde{N}}\right)(c)=0$ since $H^{*}\left(\mathcal{B}(\tilde{L})_{\tilde{N}}\right)$ is an induced representation of $\mathfrak{S}_{k}$ from a proper parabolic subgroup (cf. [Lus04]). On the other hand, if $\tilde{N}$ is regular then $\operatorname{ch} H^{*}\left(\mathcal{B}(\tilde{L})_{\tilde{N}}\right)(c)=1$. Thus we have

Lemma 6.2. Keep the notations and assumptions above. For any choice of $g_{\alpha} \in G$ such that $g_{\alpha} P \in X_{\alpha}$, we have

$$
\operatorname{ch} H^{*}\left(\mathcal{B}_{N}\right)(w)=\sum_{\alpha} \mathcal{X}\left(X_{\alpha}\right) \operatorname{ch} H^{*}\left(\mathcal{B}\left(L^{\prime}\right)_{N^{\prime}}\right)\left(w^{\prime}\right) .
$$

where the sum is over $\alpha$ such that $\tilde{N} \in$ Lie $\tilde{L}$ is regular.

This lemma is our main tool. Later we choose a suitable stratification $\mathcal{P}_{N}=\sqcup_{\alpha} X_{\alpha}$ and use Lemma 6.2 to express $\operatorname{ch} H^{*}\left(\mathcal{B}_{N}\right)(w)$ in terms of the total Springer representations associated to simple groups of rank strictly smaller than ss-rank $G$.

\section{Geometric properties for type $B$ And $C$}

In this section, we use the symbol $B($ resp. $C)$ when $G=S O_{2 n+1}(\mathbb{C})\left(\right.$ resp. $\left.G=S p_{2 n}(\mathbb{C})\right)$. Then $G$ naturally acts on $V=B \mathbb{C}^{2 n+1} C \mathbb{C}^{2 n}$ that is equipped with a nondegenerate $B$ symmetric $C$ symplectic bilinear form $\langle$,$\rangle . We identify \mathcal{B}=G / B$ with the full isotropic flag variety of $V$. Also we set $P \subset G$ in Lemma 6.2 to be a maximal parabolic subgroup such that $W_{P} \simeq \mathfrak{S}_{k} \times W^{\prime}$ for some $1 \leq k \leq n$.

Remark. Most calculations in this section are still valid for $G=S O_{2 n}(n \geq 2), 1 \leq k \leq n-2$. But we postpone this case until the next section.

7.1. $k=1$ case. This part is a reformulation of [Kim17, Section 5]. First we assume $k=1$, thus $W^{\prime}$ is the maximal parabolic subgroup of $W$ of the same type. We identify $\mathcal{P}=G / P$ with the Grassmannian of isotropic lines in $V$. Let $N \in \mathfrak{g}$ be a nilpotent element of Jordan type $\lambda=\left(1^{m_{1}} 2^{m_{2}} \cdots\right)$. First we recall vL89, Lemma 2.3.3].

Lemma 7.1. There exists an orthogonal decomposition $V=\bigoplus_{i \geq 1} V_{i}$ such that each $V_{i}$ is $N$ invariant and $\left.N\right|_{V_{i}}$ has Jordan type $\left(i^{m_{i}}\right)$. Furthermore, for a given isotropic line $l \subset \mathbb{P}($ ker $N)$ such that $\left.N\right|_{l^{\perp} / l}$ has Jordan type either $\lambda \downarrow_{(i-2)}^{(i)}$ or $\lambda \downarrow_{(i-1, i-1)}^{(i, i)}$, we may choose such a decomposition so that $l \subset V_{i}$. 


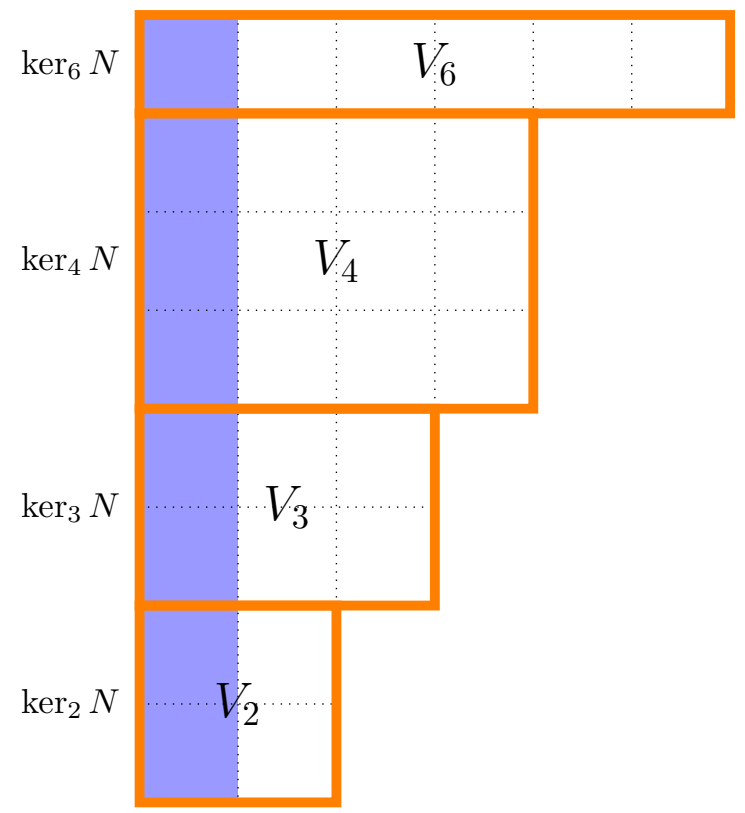

Figure 1. $V=\bigoplus_{i} V_{i}$ and $\operatorname{ker} N=\bigoplus_{i} \operatorname{ker}_{i} N$ for $G=S p_{28}(\mathbb{C})$ and $\lambda=\left(2^{2} 3^{2} 4^{3} 6^{1}\right)$ (Here each orange box is $V_{i}$ for some $i \in \mathbb{N}$, and the blue area corresponds to ker $N$.)

We fix such a decomposition and define $\operatorname{ker}_{i} N:=\operatorname{ker} N \cap V_{i}$. (See Figure1,) For $v \in \operatorname{ker} N$, we let $v_{i} \in \operatorname{ker}_{i} N$ be the projection of $v$ onto $\operatorname{ker}_{i} N$ with respect to the decomposition above so that $v=\sum_{i \geq 1} v_{i}$. The following lemma can be deduced from simple calculation with [Sho83, Section 2] or [Kim17, Section 5].

Lemma 7.2. For $0 \neq v \in \operatorname{ker} N$ such that $\langle v, v\rangle=0$, let $r \in \mathbb{N}$ be the smallest integer such that $v_{r} \neq 0$. Then the Jordan type of $\left.N\right|_{\mathbf{s p}\{v\}^{\perp} / \mathbf{s p}\{v\}}$ only depends on $\mathbf{s p}\left\{v_{r}\right\} \in \mathbb{P}\left(\operatorname{ker}_{r} N\right)$. Indeed, if the Jordan type of $N$ is $\lambda$, then $\left.N\right|_{\mathbf{s p}\{v\}^{\perp} / \mathbf{s p}\{v\}}$ has Jordan type $\lambda \downarrow_{(r-2)}^{(r)}$ or $\lambda \downarrow_{(r-1, r-1)}^{(r, r)}$. Furthermore, if $r$ is $B$ even $C$ odd, then the Jordan type of $\left.N\right|_{\mathbf{s p}\{v\}^{\perp} / \mathbf{s p}\{v\}}$ is always $\lambda \downarrow_{(r-1, r-1)}^{(r, r)}$. If $r$ is $B$ odd $C$ even, then there exists a smooth quadric hypersurface $H \subset \mathbb{P}\left(\operatorname{ker}_{r} N\right)$ such that

$$
\begin{aligned}
& \mathbf{s p}\left\{v_{r}\right\} \in H \Leftrightarrow \text { the Jordan type of }\left.N\right|_{\mathbf{s p}\{v\}^{\perp} / \mathbf{s p}\{v\}} \text { is } \lambda \downarrow_{(r-1, r-1)}^{(r, r)}, \\
& \mathbf{s p}\left\{v_{r}\right\} \notin H \Leftrightarrow \text { the Jordan type of }\left.N\right|_{\mathbf{s p}\{v\}^{\perp} / \mathbf{s p}\{v\}} \text { is } \lambda \downarrow_{(r-2)}^{(r)} .
\end{aligned}
$$

This lemma is used to prove the following proposition.

Proposition 7.3. For $\mu \vdash \operatorname{dim} V-2$, we define

$$
X_{\mu}:=\left\{l \in \mathbb{P}(\operatorname{ker} N)|\langle l, l\rangle=0, N|_{l \perp / l} \text { has Jordan type } \mu\right\} .
$$

Then $X_{\mu}$ is empty unless $\mu=\lambda \downarrow_{(r-1, r-1)}^{(r, r)}$ for some $r \geq 1$ or $\lambda \downarrow_{(r-2)}^{(r)}$ for some $r \geq 2$. Also we have

$$
\mathcal{X}\left(X_{\lambda \downarrow_{(r-1, r-1)}^{(r, r)}}\right)=2\left\lfloor\frac{m_{r}}{2}\right\rfloor, \quad \mathcal{X}\left(X_{\lambda \downarrow_{(r-2)}^{(r)}}\right)=\frac{1-(-1)^{m_{r}}}{2} .
$$


Proof. The first part is a direct consequence of Lemma 7.2. For the second claim, we first assume $r \geq 2$ and note that $X_{\lambda \downarrow_{(r-1, r-1)}^{(r, r)}} \sqcup X_{\lambda \downarrow_{(r-2)}^{(r)}}=\mathbb{P}\left(\bigoplus_{i \geq r} \operatorname{ker}_{i} N\right)-\mathbb{P}\left(\bigoplus_{i>r} \operatorname{ker}_{i} N\right)$. If $r$ is $B$ even $C$ odd then $X_{\lambda \downarrow_{(r-1, r-1)}^{(r, r)}}=\mathbb{P}\left(\bigoplus_{i \geq r} \operatorname{ker}_{i} N\right)-\mathbb{P}\left(\bigoplus_{i>r} \operatorname{ker}_{i} N\right)$ and $X_{\lambda \downarrow_{(r-2)}^{(r)}}=\emptyset$ also by Lemma 7.2, Since $m_{r}$ is always even in this case, we have

$$
\begin{gathered}
\mathcal{X}\left(X_{\lambda \downarrow \downarrow_{(r-1, r-1)}^{(r, r)}}\right)=\mathcal{X}\left(\mathbb{P}\left(\bigoplus_{i \geq r} \operatorname{ker}_{i} N\right)\right)-\mathcal{X}\left(\mathbb{P}\left(\bigoplus_{i>r} \operatorname{ker}_{i} N\right)\right)=m_{r}=2\left\lfloor\frac{m_{r}}{2}\right\rfloor, \\
\mathcal{X}\left(X_{\lambda \downarrow_{(r-2)}^{(r)}}\right)=0=\frac{1-(-1)^{m_{r}}}{2},
\end{gathered}
$$

thus the result follows.

Now suppose $r$ is $B$ odd $C$ even. We have a projection map

$$
\phi_{r}: \mathbb{P}\left(\bigoplus_{i \geq r} \operatorname{ker}_{i} N\right)-\mathbb{P}\left(\bigoplus_{i>r} \operatorname{ker}_{i} N\right) \rightarrow \mathbb{P}\left(\operatorname{ker}_{r} N\right): \mathbf{s p}\{v\} \mapsto \mathbf{s p}\left\{v_{r}\right\} .
$$

which is a well-defined affine bundle with fiber isomorphic to $\bigoplus_{i>r} \operatorname{ker}_{i} N$. As $\phi_{r}\left(X_{\lambda \downarrow_{(r-1, r-1)}^{(r, r)}}\right)=$ $H$ and $\phi_{r}\left(X_{\lambda \downarrow_{(r-2)}^{(r)}}\right)=\mathbb{P}\left(\operatorname{ker}_{r} N\right)-H$ where $H$ is as in (7.1), $\mathcal{X}\left(X_{\lambda \downarrow_{(r-1, r-1)}^{(r, r)}}\right)=\mathcal{X}(H)$ and $\mathcal{X}\left(X_{\lambda \downarrow_{(r-2)}^{(r)}}\right)=\mathcal{X}\left(\mathbb{P}\left(\operatorname{ker}_{r} N\right)\right)-\mathcal{X}(H)$. Then we use the fact that the Euler characteristic of a smooth quadric hypersurface in $\mathbb{P}^{n-1}$ is $2\left\lfloor\frac{n}{2}\right\rfloor$. If $r=1$, then we argue similarly with the projection

$$
\phi_{1}: X_{\lambda \downarrow}^{(1,1)} \rightarrow \mathbb{P}\left(\operatorname{ker}_{1} N\right): \mathbf{s p}\{v\} \mapsto \mathbf{s p}\left\{v_{1}\right\}
$$

whose image is $H \subset \mathbb{P}\left(\operatorname{ker}_{1} N\right)$ defined in (7.1).

From this proposition and Lemma 6.2 we deduce the following result.

Proposition 7.4. Let $W^{\prime} \subset W$ be the maximal parabolic subgroup of the same type. For $\lambda=$ $\left(1^{m_{1}} 2^{m_{2}} \ldots\right)$ we have

$$
\operatorname{Res}_{W^{\prime}}^{W} \operatorname{ch} \mathbf{T S p}(\lambda)=\sum_{i \geq 1, m_{i} \geq 2} 2\left\lfloor\frac{m_{i}}{2}\right\rfloor \operatorname{ch} \mathbf{T S p}\left(\lambda \downarrow_{(i-1, i-1)}^{(i, i)}\right)+\sum_{i \geq 2, m_{i} \text { odd }} \operatorname{ch} \mathbf{T S p}\left(\lambda \downarrow_{(i-2)}^{(i)}\right) .
$$

Here $\mathbf{T S p}\left(\lambda \downarrow_{(i-1, i-1)}^{(i, i)}\right), \mathbf{T S p}\left(\lambda \downarrow_{(i-2)}^{(i)}\right)$ are total Springer representations of $W^{\prime}$.

7.2. $k=2$ case. Here, we assume $k=2$ and identify $\mathcal{P}=G / P$ with the Grassmannian of twodimensional isotropic subspaces in $V$. (This is a crucial step for generalizing our statement to the case for arbitrary $k$.) We also assume that an isotropic line $l \subset \operatorname{ker} N$ is given and that $\left.N\right|_{l^{\perp} / l}$ has Jordan type $\lambda \downarrow_{(i-2)}^{(i)}$ or $\lambda \downarrow_{(i-1, i-1)}^{(i, i)}$ for some fixed $i \geq 2$. (The reason to exclude $i=1$ case will be apparent when we state Proposition [7.7) We use Lemma 7.1 to fix a decomposition $V=\bigoplus_{j \geq 1} V_{j}$ such that $l \subset V_{i}$. We write $\widetilde{N}:=\left.N\right|_{l^{\perp} / l}$ and denote:

- Case $A$. if $\widetilde{N}$ has Jordan type $\lambda \downarrow_{(i-2)}^{(i)}$, and

- Case B. if $\widetilde{N}$ has Jordan type $\lambda \downarrow_{(i-1, i-1)}^{(i, i)}$.

We start with improving Lemma 7.1. (See Figure 2) 


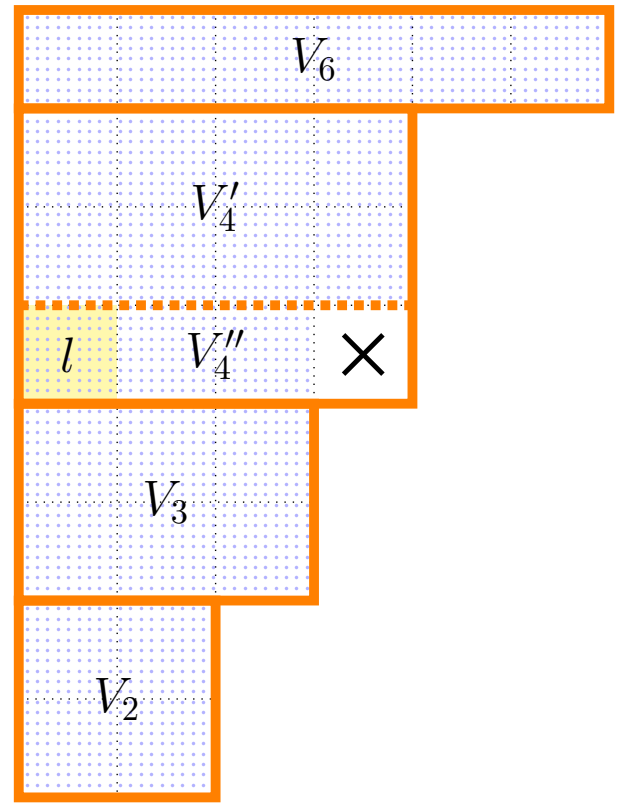

(A) Case $A, i=4$

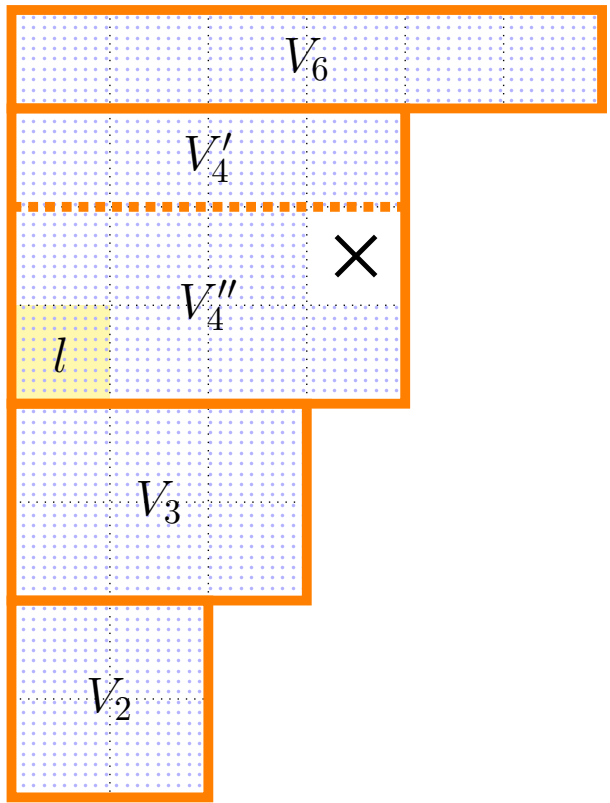

(в) Case $B, i=4$

Figure 2. $V=\left(\bigoplus_{j \neq i} V_{j}\right) \oplus V_{i}^{\prime} \oplus V_{i}^{\prime \prime}$ for $G=S p_{28}(\mathbb{C})$ and $\lambda=\left(2^{2} 3^{2} 4^{3} 6^{1}\right)$

(Here the yellow box is $l$ and the blue-dotted area corresponds to $l^{\perp}$.)

Lemma 7.5. Keep the assumption in Lemma 7.1 and suppose $l \subset V_{i}$.

- Case $A$. There exists an orthogonal decomposition $V_{i}=V_{i}^{\prime} \oplus V_{i}^{\prime \prime}$ such that

- $V_{i}^{\prime}$ and $V_{i}^{\prime \prime}$ are $N$-invariant,

- $\left.N\right|_{V_{i}^{\prime}},\left.N\right|_{V_{i}^{\prime \prime}}$ have Jordan types $\left(i^{m_{i}-1}\right),\left(i^{1}\right)$, respectively, and

- $l \subset V_{i}^{\prime \prime}$. (In particular, $\left.l=\operatorname{ker} N \cap V_{i}^{\prime \prime}\right)$

Thus in particular, $V_{i}^{\prime} \perp l$ and $\left.N\right|_{\left(l^{\perp} \cap V_{i}^{\prime \prime}\right) / l}$ has Jordan type $\left((i-2)^{1}\right)$.

- Case B. There exists an orthogonal decomposition $V_{i}=V_{i}^{\prime} \oplus V_{i}^{\prime \prime}$ such that

- $V_{i}^{\prime}$ and $V_{i}^{\prime \prime}$ are $N$-invariant,

- $\left.N\right|_{V_{i}^{\prime}},\left.N\right|_{V_{i}^{\prime \prime}}$ have Jordan types $\left(i^{m_{i}-2}\right),\left(i^{2}\right)$, respectively, and

- $l \subset V_{i}^{\prime \prime}$.

Thus in particular, $V_{i}^{\prime} \perp l$ and $\left.N\right|_{\left(l \perp \cap V_{i}^{\prime \prime}\right) / l}$ has Jordan type $\left((i-1)^{2}\right)$.

Proof. It suffices to consider the case when $\lambda=\left(i^{m_{i}}\right)$ which we assume here. First assume Case $A$. Then we have an orthogonal decomposition $l^{\perp} / l=\widetilde{V_{i}} \oplus \widetilde{V_{i-2}}$ which satisfies the property in Lemma 7.1 with respect to $\widetilde{N}$. Now take the inverse image of $\widetilde{V}_{i}$ under the map $l^{\perp} \rightarrow l^{\perp} / l$, say $W_{i} \subset \mathbb{C}^{2 n}$, 
and take a complementary space $V_{i}^{\prime}$ of $l$ in $W_{i}$. We also set $V_{i}^{\prime \prime}=\left(V_{i}^{\prime}\right)^{\perp}$. Then it is easy to see that they satisfy the desired properties. Case $B$ is also similar.

We want to have a similar decomposition of $l^{\perp} / l$ similar to Lemma 7.1 with respect to $\tilde{N}$, in accordance with $V=\left(\bigoplus_{j \neq i} V_{j}\right) \oplus V_{i}^{\prime} \oplus V_{i}^{\prime \prime}$. The following lemma is an easy exercise.

Lemma 7.6. Keep the assumptions above.

- Case $A$. The decomposition $l^{\perp} / l=\bigoplus_{j \geq 1} \widetilde{V_{j}}$ where

$\widetilde{V_{j}}=\left(V_{j} \oplus l\right) / l$ for $j \neq i, i-2, \quad \widetilde{V_{i}}=\left(V_{i}^{\prime} \oplus l\right) / l, \quad \widetilde{V_{i-2}}=\left(\left(V_{i}^{\prime \prime} \cap l^{\perp}\right) \oplus V_{i-2}\right) / l$

gives the decomposition of $l^{\perp} / l$ which satisfies the criteria in Lemma 7.1 with respect to $\tilde{N}$.

- Case B. The decomposition $l^{\perp} / l=\bigoplus_{j \geq 1} \widetilde{V_{j}}$ where

$\widetilde{V_{j}}=\left(V_{j} \oplus l\right) / l$ for $j \neq i, i-1, \quad \widetilde{V_{i}}=\left(V_{i}^{\prime} \oplus l\right) / l, \quad \widetilde{V_{i-1}}=\left(\left(V_{i}^{\prime \prime} \cap l^{\perp}\right) \oplus V_{i-1}\right) / l$

gives the decomposition of $l^{\perp} / l$ which satisfies the criteria in Lemma 7.1 with respect to $\tilde{N}$.

Previously we defined $\operatorname{ker}_{j} N:=\operatorname{ker} N \cap V_{j}$. Similarly, we also let $\operatorname{ker}_{i}^{\prime} N:=\operatorname{ker} N \cap V_{i}^{\prime}, \operatorname{ker}_{i}^{\prime \prime}:=$ $\operatorname{ker} N \cap V_{i}^{\prime \prime}$ and $\operatorname{ker}_{j} \widetilde{N}:=\operatorname{ker} \widetilde{N} \cap \widetilde{V}_{j}$. Then it is easy to see that

- Case $A$.

$$
\begin{gathered}
\operatorname{ker}_{j} \widetilde{N}=\left(\operatorname{ker}_{j} N \oplus l\right) / l \text { for } j \neq i, i-2, \\
\operatorname{ker}_{i} \widetilde{N}=\left(\operatorname{ker}_{i}^{\prime} N \oplus l\right) / l, \quad \operatorname{ker}_{i-2} \widetilde{N}=\left(\left(N^{-1} l \cap V_{i}^{\prime \prime}\right) \oplus V_{i-2}\right) / l
\end{gathered}
$$

- Case $B$.

$$
\begin{gathered}
\operatorname{ker}_{j} \widetilde{N}=\left(\operatorname{ker}_{j} N \oplus l\right) / l \text { for } j \neq i, i-1, \\
\operatorname{ker}_{i} \widetilde{N}=\left(\operatorname{ker}_{i}^{\prime} N \oplus l\right) / l, \quad \operatorname{ker}_{i-1} \widetilde{N}=\left(\left(N^{-1} l \cap V_{i}^{\prime \prime}\right) \oplus V_{i-1}\right) / l .
\end{gathered}
$$

From now on we denote by $\bar{v}:=v+l \in V / l$ the image of $v \in V$ under the projection $V \rightarrow V / l$. We choose $v_{0} \in N^{-1} l \cap V_{i}^{\prime \prime} \operatorname{such}$ that $\operatorname{sp}\left\{N v_{0}\right\}=l$. For any $v \in \operatorname{ker} N$, we write $\tilde{v}:=\overline{v_{0}+v}$. Also we let $v=\sum_{j \geq 1} v_{j}$ such that $v_{j} \in \operatorname{ker}_{j} N$ and let $v_{i}=v_{i}^{\prime}+v_{i}^{\prime \prime}$ where $v_{i}^{\prime} \in \operatorname{ker}_{i}^{\prime} N$ and $v_{i}^{\prime \prime} \in \operatorname{ker}_{i}^{\prime \prime} N$. (See Figure [3]) Then $\tilde{v}=\sum_{j \geq 1} \tilde{v}_{j}, \tilde{v}_{j} \in \operatorname{ker}_{j} \widetilde{N}$ where

- Case $A . \tilde{v}_{j}=\overline{v_{j}}$ for $j \neq i-2$ (in particular, $\tilde{v}_{i}=\overline{v_{i}^{\prime}}=\overline{v_{i}}$ ) $\quad \tilde{v}_{i-2}=\overline{v_{0}+v_{i-2}}$,

- Case B. $\tilde{v}_{j}=\overline{v_{j}}$ for $j \neq i, i-1, \quad \tilde{v}_{i}=\overline{v_{i}^{\prime}}, \quad \tilde{v}_{i-1}=\overline{v_{0}+v_{i}^{\prime \prime}+v_{i-1}}$.

Let $r, r^{\prime} \in \mathbb{N}$ be the smallest integers such that $v_{r} \neq 0$ and $\tilde{v}_{r^{\prime}} \neq 0$. Then from the observation above it is clear that

- Case A. $r^{\prime}=\min (r, i-2)$.

- Case B. $r^{\prime}=\min (r, i-1)$.

Therefore, for $\mathcal{V} \subset V$ such that $\operatorname{dim} \mathcal{V}=2$ and $N \mathcal{V}=l \subset \mathcal{V}$, using Lemma 7.2 we have 


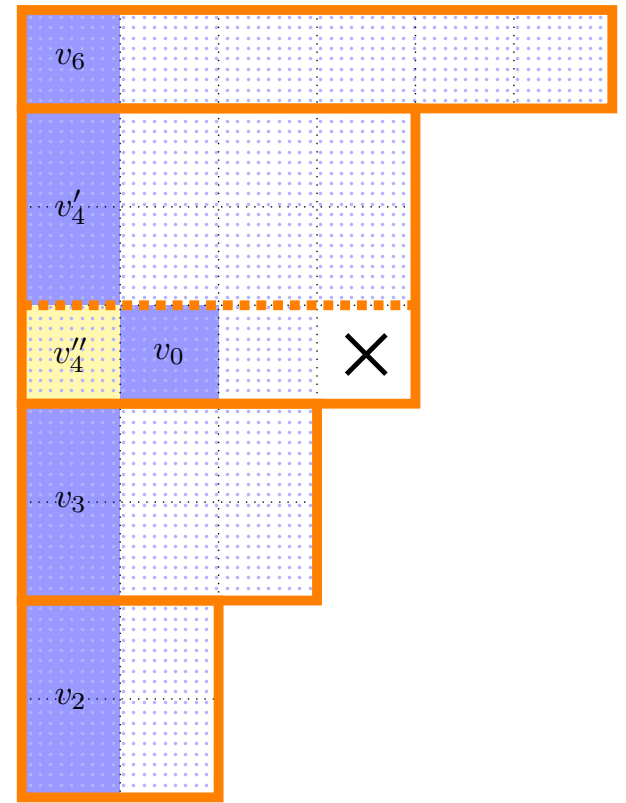

(A) Case $A, i=4$

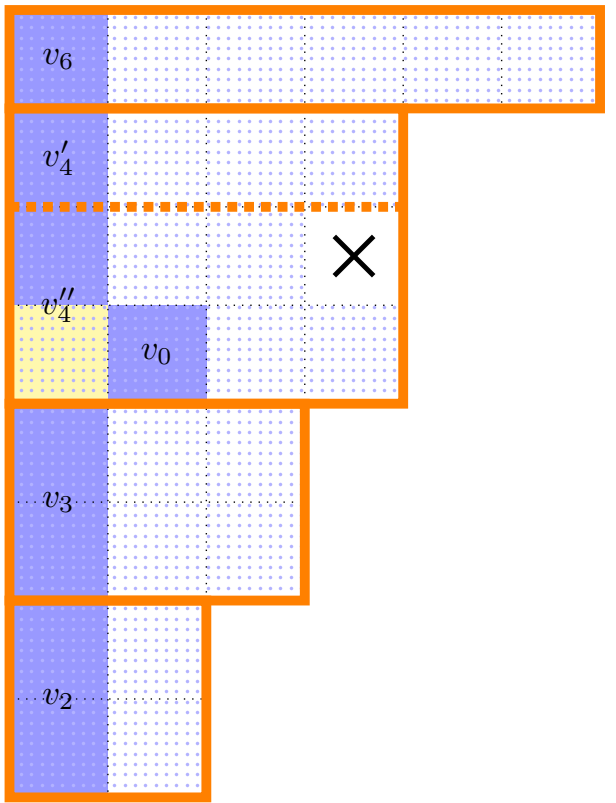

(в) Case $B, i=4$

Figure 3. $v=\left(\sum_{j \neq i} v_{j}\right)+v_{i}^{\prime}+v_{i}^{\prime \prime}$ and $v_{0} \in N^{-1} l$ for $G=S p_{28}(\mathbb{C})$ and $\lambda=\left(2^{2} 3^{2} 4^{3} 6^{1}\right)$

(Here the yellow box is $l$ and the blue area corresponds to ker $\widetilde{N}$.)

- Case $A$. the Jordan type of $\left.N\right|_{\mathcal{V} \perp / \mathcal{V}}$ is either

$$
\lambda \downarrow_{(i-4)}^{(i)}, \quad \lambda \downarrow_{(i-3, i-3)}^{(i, i-2)}, \quad \lambda \downarrow_{(i-2, r-2)}^{(i, r)}, \quad \text { or } \lambda \downarrow_{(i-2, r-1, r-1)}^{(i, r, r)},
$$

for some $r<i-2$.

- Case B. the Jordan type of $\left.N\right|_{\mathcal{V} \perp / \mathcal{V}}$ is either

$$
\lambda \downarrow_{(i-1, i-3)}^{(i, i)}, \quad \lambda \downarrow_{(i-2, i-2)}^{(i, i)}, \quad \lambda \downarrow_{(i-1, i-1, r-2)}^{(i, i, r)}, \quad \text { or } \lambda \downarrow_{(i-1, i-1, r-1, r-1)}^{(i, i, r, r)},
$$

for some $r<i-1$.

Now we state a key step to calculate the formula in Lemma 6.2 for $k=2$ case.

Proposition 7.7. Let $l \in \mathbb{P}(\operatorname{ker} N)$ be an isotropic line and define

$$
X_{\mu, l}:=\left\{\mathcal{V} \subset V|\langle\mathcal{V}, \mathcal{V}\rangle=0, \operatorname{dim} \mathcal{V}=2, N \mathcal{V}=l \subset \mathcal{V}, N|_{\mathcal{V} \perp / \mathcal{V}} \text { has Jordan type } \mu\right\}
$$

- Case A. We have

$$
\begin{aligned}
& \text { (a) } \mathcal{X}\left(X_{\mu, l}\right)=1-(-1)^{m_{i-2}} \text { if } \mu=\lambda \downarrow_{(i-3, i-3)}^{(i, i-2)} \text { for } i \geq 3 \text {, } \\
& \text { (b) } \mathcal{X}\left(X_{\mu, l}\right)=(-1)^{m_{i-2}} \text { if } \mu=\lambda \downarrow_{(i-4)}^{(i)} \text { for } i \geq 4 \text {, and } \\
& \text { (c) } \mathcal{X}\left(X_{\mu, l}\right)=0 \text { otherwise. }
\end{aligned}
$$

- Case B. We have 


$$
\begin{aligned}
& \text { (a) } \mathcal{X}\left(X_{\mu, l}\right)=1 \text { if } \mu=\lambda \downarrow_{(i-2, i-2)}^{(i, i)} \text { for } i \geq 2, \\
& \text { (b) } \mathcal{X}\left(X_{\mu, l}\right)=0 \text { otherwise. }
\end{aligned}
$$

Remark. If $i=1$, then there does not exist any $\mathcal{V} \in V$ such that $N \mathcal{V}=l \subset \mathcal{V}$. Thus it is enough to assume $i \geq 2$ as we did at the beginning.

Proof. First we consider Case $A(c)$. For $\mu$ different from $\lambda \downarrow_{(i-3, i-3)}^{(i, i-2)}$ and $\lambda \downarrow_{(i-4)}^{(i)}$, it suffices to assume that $\mu=\lambda \downarrow_{(i-2, r-2)}^{(i, r)}$ or $\lambda \downarrow_{(i-2, r-1, r-1)}^{(i, r, r)}$ for some $1 \leq r<i-2$. Moreover, it is enough to assume that $i \geq 4$. We briefly write $\mu^{1}=\lambda \downarrow_{(i-2, r-2)}^{(i, r)}$ and $\mu^{2}=\lambda \downarrow_{(i-2, r-1, r-1)}^{(i, r, r)}$.

We can choose $v_{0} \in N^{-1} l \cap V_{i}^{\prime \prime}$ such that $\mathbf{s p}\left\{N v_{0}\right\}=l$. Then the map

$$
\psi: \operatorname{ker} N \rightarrow\{\mathcal{V} \subset V \mid\langle\mathcal{V}, \mathcal{V}\rangle=0, \operatorname{dim} \mathcal{V}=2, N \mathcal{V}=l \subset \mathcal{V}\}: v \mapsto \operatorname{sp}\left\{v_{0}+v\right\} \oplus l
$$

is an affine bundle with fiber $l$. By Lemma 7.2, we have

$$
\psi(v) \in X_{\mu^{1}, l} \sqcup X_{\mu^{2}, l} \Leftrightarrow \tilde{v} \in \bigoplus_{j \geq r} \operatorname{ker}_{j} \tilde{N}-\bigoplus_{j>r} \operatorname{ker}_{j} \tilde{N} \Leftrightarrow v \in \bigoplus_{j \geq r} \operatorname{ker}_{j} N-\bigoplus_{j>r} \operatorname{ker}_{j} N
$$

where $\tilde{v}=\overline{v_{0}+v}$. (The condition $\langle\psi(v), \psi(v)\rangle=0$ is automatic for $i \geq 4$.) Thus we have

$$
\psi^{-1}\left(X_{\mu^{1}, l} \sqcup X_{\mu^{2}, l}\right)=\bigoplus_{j \geq r} \operatorname{ker}_{j} N-\bigoplus_{j>r} \operatorname{ker}_{j} N .
$$

For such $r$, consider

$$
\phi_{r}: \bigoplus_{j \geq r} \operatorname{ker}_{j} N-\bigoplus_{j>r} \operatorname{ker}_{j} N \rightarrow \mathbb{P}\left(\operatorname{ker}_{r} \tilde{N}\right): v \mapsto \mathbf{s p}\left\{\tilde{v}_{r}\right\} .
$$

This map is a fiber bundle with fiber isomorphic to $\left(\bigoplus_{j>r} \operatorname{ker}_{j} N\right) \times\left(\mathbb{A}^{1}-\{0\}\right)$. As the Jordan type of $\left.N\right|_{\psi(v)^{\perp} / \psi(v)}$ is determined by $\mathbf{s p}\left\{\tilde{v}_{r}\right\} \in \mathbb{P}\left(\operatorname{ker}_{r} \widetilde{N}\right)$, there exists $Y_{\mu^{1}, l}, Y_{\mu^{2}, l} \subset \mathbb{P}\left(\operatorname{ker}_{r} \tilde{N}\right)$ such that

$$
\phi_{r}^{-1}\left(Y_{\mu^{1}, l}\right)=\psi^{-1}\left(X_{\mu^{1}, l}\right), \quad \phi_{r}^{-1}\left(Y_{\mu^{2}, l}\right)=\psi^{-1}\left(X_{\mu^{2}, l}\right) .
$$

But since the Euler characteristic of a fiber of $\phi_{r}$ is 0 , it follows that $\mathcal{X}\left(X_{\mu^{1}}\right)=\mathcal{X}\left(\psi^{-1}\left(X_{\mu^{1}, l}\right)\right)=$ $\mathcal{X}\left(\phi_{r}^{-1}\left(Y_{\mu^{1}, l}\right)\right)=0$ and similarly $\mathcal{X}\left(X_{\mu^{2}, l}\right)=0$. This proves Case $A(c)$. Case $B(b)$ when $\mu \neq$ $\lambda \downarrow_{(i-1, i-3)}^{(i, i)}$ is also similar.

Now consider Case $A(a)$ and $A(b)$, thus it is enough to assume that $i \geq 3$. Then $\tilde{v}_{i-2}=\overline{v_{0}+v_{i-2}}$ is always nonzero. This time we set $\mu^{1}=\lambda \downarrow_{(i-3, i-3)}^{(i, i-2)}$ and $\mu^{2}=\lambda \downarrow_{(i-4)}^{(i)}$. Here we only deal with the case when $i \geq 4$, but $i=3$ case is totally analogous. (If $i=3, \mu^{2}$ is not well-defined and one needs to be careful about the condition $\langle\psi(v), \psi(v)\rangle=0$ which is automatic for $i \geq 4$.)

First we observe the following. (Also see Figure 4.)

Lemma 7.8. $\mathcal{X}\left(X_{\mu^{1}, l}\right)$ and $\mathcal{X}\left(X_{\mu^{2}, l}\right)$ become unchanged if we put $V_{j}=0$ for $j \neq i, i-2$ and $V_{i}^{\prime}=0$, i.e. if $V=V_{i}^{\prime \prime} \oplus V_{i-2}$.

Proof of Lemma 7.8. Again we define

$$
\psi: \operatorname{ker} N \rightarrow\{\mathcal{V} \subset V \mid\langle\mathcal{V}, \mathcal{V}\rangle=0, \operatorname{dim} \mathcal{V}=2, N \mathcal{V}=l \subset \mathcal{V}\}: v \mapsto \mathbf{s p}\left\{v_{0}+v\right\} \oplus l
$$


Then similarly to above we have

$$
\psi^{-1}\left(X_{\mu^{1}, l} \sqcup X_{\mu^{2}, l}\right)=\bigoplus_{j \geq i-2} \operatorname{ker}_{j} N .
$$

Also we let

$$
\phi^{\prime}: \bigoplus_{j \geq i-2} \operatorname{ker}_{j} N \rightarrow \mathbb{P}\left(\operatorname{ker}_{i-2} \tilde{N}\right): v \mapsto \mathbf{s p}\left\{\tilde{v}_{i-2}\right\} .
$$

The image of $\phi^{\prime}$ is $\mathbb{P}\left(\operatorname{ker}_{i-2} \tilde{N}\right)-\mathbb{P}\left(\left(\operatorname{ker}_{i-2} N \oplus l\right) / l\right)$ which is isomorphic to an affine space, and when restricted to the image $\phi^{\prime}$ is a fiber bundle with fiber isomorphic to $\bigoplus_{j>i-2} \operatorname{ker}_{j} N$. Also by the same reason as above there exists $Y_{\mu^{1}, l}, Y_{\mu^{2}, l} \subset \mathbb{P}\left(\operatorname{ker}_{i-2} \tilde{N}\right)$ such that

$$
\phi^{\prime-1}\left(Y_{\mu^{1}, l}\right)=\psi^{-1}\left(X_{\mu^{1}, l}\right), \quad \phi^{\prime-1}\left(Y_{\mu^{2}, l}\right)=\psi^{-1}\left(X_{\mu^{2}, l}\right)
$$

Then $\mathcal{X}\left(X_{\mu^{1}, l}\right)=\mathcal{X}\left(Y_{\mu^{1}, l}\right)$ and $\mathcal{X}\left(X_{\mu^{2}, l}\right)=\mathcal{X}\left(Y_{\mu^{2}, l}\right)$. We recall the orthogonal decomposition $V=\left(\bigoplus_{j \neq i} V_{j}\right) \oplus V_{i}^{\prime} \oplus V_{i}^{\prime \prime}$. It is clear that the varieties $Y_{\mu^{1}, l}, Y_{\mu^{2}, l}$ only depend (up to isomorphism) on the restriction of $N$ to $V_{i}^{\prime \prime} \oplus V_{i-2}$ and $l \subset V_{i}^{\prime \prime}$. As $\mathcal{X}\left(X_{\mu^{1}, l}\right)=\mathcal{X}\left(Y_{\mu^{1}, l}\right)$ and $\mathcal{X}\left(X_{\mu^{2}, l}\right)=\mathcal{X}\left(Y_{\mu^{2}, l}\right)$, the claim follows.

In other words, we may assume that $N$ has Jordan type $\lambda=\left((i-2)^{m_{i-2}} i^{1}\right)$ and $l \subset \operatorname{ker}_{i} N$. Note that $i$ should be $B$ odd $C$ even in this case. Following the idea of [SS70, E.93], we choose vectors $e_{0}, e_{1}, \cdots, e_{m_{i-2}} \in V$ that satisfy the following.

- $\left\{N^{j} e_{0} \mid 0 \leq j \leq i-1\right\} \cup\left\{N^{j^{\prime}} e_{p} \mid 0 \leq j^{\prime} \leq i-3,1 \leq p \leq m_{i-2}\right\}$ is a basis of $V$.

- The value of $\langle$,$\rangle on any pair of the elements above are zero except$

$$
(-1)^{j}\left\langle N^{j} e_{0}, N^{i-1-j} e_{0}\right\rangle=(-1)^{j^{\prime}}\left\langle N^{j^{\prime}} e_{p}, N^{i-3-j^{\prime}} e_{p}\right\rangle=1
$$

for $0 \leq j \leq i-1,0 \leq j^{\prime} \leq i-3,1 \leq p \leq m_{i-2}$.

- $l=\mathbf{s p}\left\{N^{i-1} e_{0}\right\}$.

Also we may assume that $v_{0}=N^{i-2} e_{0}$. Then $\psi$ induces an isomorphism of varieties

$$
\left.\psi\right|_{\operatorname{ker}_{i-2} N}: \operatorname{ker}_{i-2} N \rightarrow\{\mathcal{V} \subset V \mid\langle\mathcal{V}, \mathcal{V}\rangle=0, \operatorname{dim} \mathcal{V}=2, N \mathcal{V}=l \subset \mathcal{V}\}: v \mapsto \mathbf{s p}\left\{v_{0}+v\right\} \oplus l
$$

We identify $\operatorname{ker}_{i-2} N$ with $\mathbb{A}^{m_{i-2}}$ using the basis $\left\{N^{i-3} e_{p}\right\}_{1 \leq p \leq m_{i-2}}$. Then by direct calculation, for any $v \in \operatorname{ker}_{i-2} N$ such that $v=\sum_{p=1}^{m_{i-2}} a_{p} N^{i-3} e_{p}$ we have (see also [Sho83, p.249])

$$
\psi(v) \in X_{\mu^{1}, l} \Leftrightarrow 1+a_{1}^{2}+\cdots+a_{m_{i-2}}^{2}=0, \quad \psi(v) \in X_{\mu^{2}, l} \Leftrightarrow 1+a_{1}^{2}+\cdots+a_{m_{i-2}}^{2} \neq 0 .
$$

But for any $m \in \mathbb{N}$ it is easy to see that

$$
\mathcal{X}\left(\left\{\left(a_{1}, \cdots, a_{m}\right) \in \mathbb{A}^{m} \mid a_{1}^{2}+\cdots+a_{m}^{2}+1=0\right\}\right)=1-(-1)^{m} .
$$

Therefore we have $\mathcal{X}\left(X_{\mu^{1}, l}\right)=1-(-1)^{m_{i-2}}$ and $\mathcal{X}\left(X_{\mu^{2}, l}\right)=\mathcal{X}\left(\mathbb{A}^{m_{i-2}}\right)-\mathcal{X}\left(X_{\mu^{1}, l}\right)=(-1)^{m_{i-2}}$.

We proceed with Case $B(a)$ and $B(b)$ for $i \geq 2$ which are similar to above. Again $\tilde{v}_{i-1}=$ $\overline{v_{0}+v_{i-1}}$ is always nonzero. This time we set $\mu^{1}=\lambda \downarrow_{(i-2, i-2)}^{(i, i)}$ and $\mu^{2}=\lambda \downarrow_{(i-1, i-3)}^{(i, i)}$. Here we only deal with the case when $i \geq 3$, but $i=2$ case is totally analogous. (If $i=2$, $\mu^{2}$ is not well-defined and one needs to be careful about the condition $\langle\psi(v), \psi(v)\rangle=0$, which is automatic when $i \geq 3$.) 

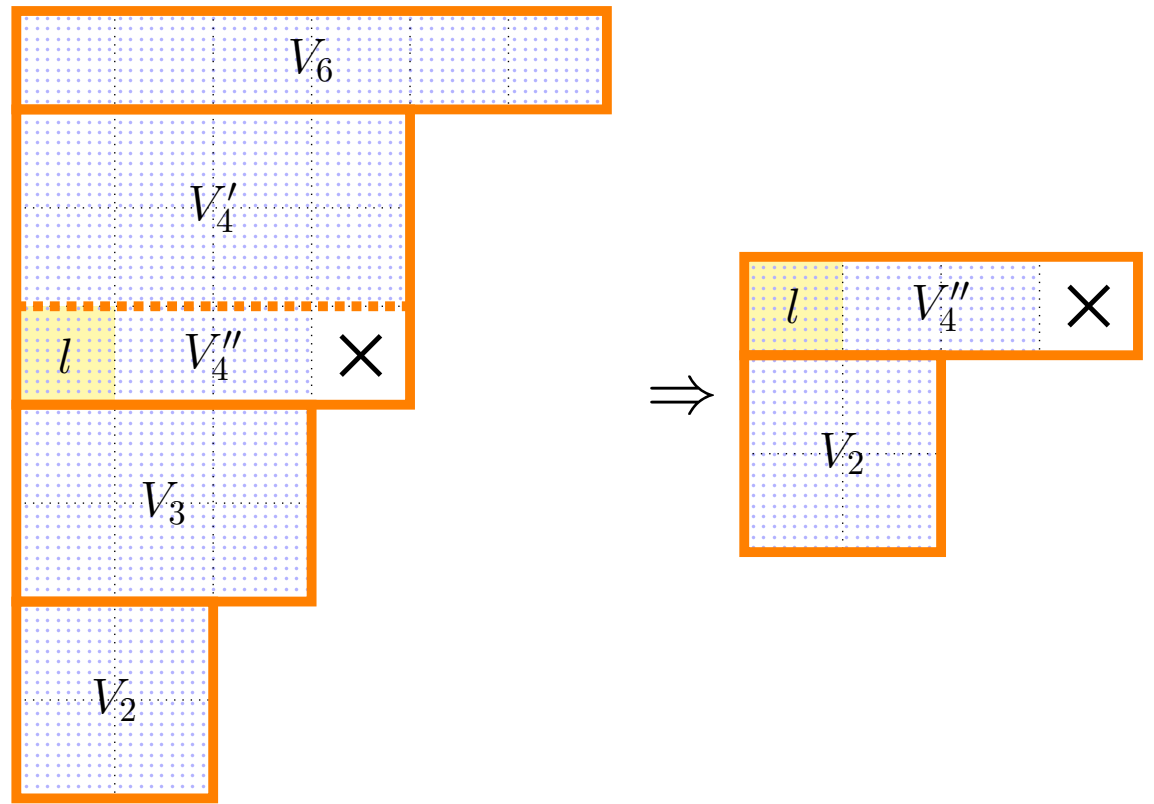

Figure 4. Setting $V_{6}=V_{4}^{\prime}=V_{3}=0$ in Case $A, i=4$ for $G=S p_{28}(\mathbb{C})$ and $\lambda=\left(2^{2} 3^{2} 4^{3} 6^{1}\right)$

First note that we may put $V_{j}=0$ for $j \neq i, i-1$ and $V_{i}^{\prime}=0$, which can be similarly justified to Lemma 7.8 Therefore, it suffices to consider the case when $\lambda=\left((i-1)^{m_{i-1}} i^{2}\right)$ and $l \subset \operatorname{ker}_{i} N$. If $i$ is $B$ odd $C$ even, then for any line $\tilde{l} \subset \operatorname{ker}_{i-1} \tilde{N},\left.\widetilde{N}\right|_{\tilde{l} \perp / \tilde{l}}$ has Jordan type $\left(\lambda \downarrow_{(i-1, i-1)}^{(i, i)}\right) \downarrow_{(i-2, i-2)}^{(i-1, i-1)}=$ $\lambda \downarrow_{(i-2, i-2)}^{(i, i)}$ by Lemma 7.2. Thus $X_{\mu^{2}, l}=\emptyset$ and $\psi(\operatorname{ker} N)=X_{\mu^{1}, l}$, i.e.

$$
\psi: \operatorname{ker} N \rightarrow X_{\mu^{1}, l}: v \mapsto \mathbf{s p}\left\{v_{0}+v\right\} \oplus l .
$$

is an affine bundle with fiber $l$. Thus

$$
\mathcal{X}\left(X_{\mu^{1}, l}\right)=\mathcal{X}(\operatorname{ker} N)=1
$$

which proves the claim in this case.

Now we assume that $i$ is $B$ even $C$ odd. Using the argument in [SS70, E.93], we choose $f_{0}, g_{0}, e_{1}, \cdots, e_{m_{i-1}}$ such that the following conditions hold.

- $\left\{N^{j} f_{0} \mid 0 \leq j \leq i-1\right\} \cup\left\{N^{j} g_{0} \mid 0 \leq j \leq i-1\right\} \cup\left\{N^{j^{\prime}} e_{p} \mid 0 \leq j^{\prime} \leq i-2,1 \leq p \leq m_{i-1}\right\}$ is a basis of $V$.

- The value of $\langle$,$\rangle on any pair of the elements above is zero except$

$$
(-1)^{j}\left\langle N^{j} f_{0}, N^{i-1-j} g_{0}\right\rangle=\epsilon(-1)^{j}\left\langle N^{i-1-j} g_{0}, N^{i} f_{0}\right\rangle=(-1)^{j^{\prime}}\left\langle N^{j^{\prime}} e_{p}, N^{i-2-j^{\prime}} e_{p}\right\rangle=1
$$

for $0 \leq j \leq i-1,1 \leq p \leq m_{i-1}, 0 \leq j^{\prime} \leq i-2$. (Here $\epsilon$ equals $B 1 \square-1$.)

- $l=\mathbf{s p}\left\{N^{i-1} f_{0}\right\}$. 
Also we may assume that $v_{0}=N^{i-2} f_{0}$. Then $\psi$ induces an isomorphism of varieties

$$
\begin{aligned}
\left.\psi\right|_{\operatorname{ker}_{i-1} N \oplus \mathbf{s p}\left\{N^{i-1} g_{0}\right\}} & : \operatorname{ker}_{i-1} N \oplus \mathbf{s p}\left\{N^{i-1} g_{0}\right\} \rightarrow\{\mathcal{V} \subset V \mid\langle\mathcal{V}, \mathcal{V}\rangle=0, \operatorname{dim} \mathcal{V}=2, N \mathcal{V}=l \subset \mathcal{V}\} \\
& : v \mapsto \mathbf{s p}\left\{v_{0}+v\right\} \oplus l .
\end{aligned}
$$

We identify $\operatorname{ker}_{i-1} N \oplus \mathbf{s p}\left\{N^{i-1} g_{0}\right\}$ with $\mathbb{A}^{m_{i-1}+1}$ using the basis $\left\{N^{i-2} e_{p}\right\}_{1 \leq p \leq m_{i-2}} \cup\left\{N^{i-1} g_{0}\right\}$. Then by direct calculation, for any $v \in \operatorname{ker}_{i-1} N \oplus \mathbf{s p}\left\{N^{i-1} g_{0}\right\}$ such that $v=\sum_{p=1}^{m_{i-1}} a_{p} N^{i-2} e_{p}+$ $b N^{i-1} g_{0}$ we have

$$
\psi(v) \in X_{\mu^{1}, l} \Leftrightarrow 2 b+a_{1}^{2}+\cdots+a_{m_{i-2}}^{2}=0, \quad \psi(v) \in X_{\mu^{2}, l} \Leftrightarrow 2 b+a_{1}^{2}+\cdots+a_{m_{i-2}}^{2} \neq 0 .
$$

But it is easy to see that for $m \in \mathbb{N}$ we have

$$
\mathcal{X}\left(\left\{\left(b, a_{1}, \cdots, a_{m}\right) \in \mathbb{A}^{m+1} \mid a_{1}^{2}+\cdots+a_{m}^{2}+2 b=0\right\}\right)=1 .
$$

Therefore we have $\mathcal{X}\left(X_{\mu^{1}, l}\right)=1$ and $\mathcal{X}\left(X_{\mu^{2}, l}\right)=\mathcal{X}\left(\mathbb{A}^{m_{i-1}+1}\right)-\mathcal{X}\left(X_{\mu^{1}, l}\right)=0$. It completes the proof of the proposition.

We combine Proposition 7.3 and 7.7. For $\mu \vdash \operatorname{dim} V-4$, define

$$
X_{\mu}:=\left\{\mathcal{V} \subset V|\langle\mathcal{V}, \mathcal{V}\rangle=0, \operatorname{dim} \mathcal{V}=2, N \mathcal{V} \subset \mathcal{V}, N|_{\mathcal{V}} \text { is regular, }\left.N\right|_{\mathcal{V}^{\perp} / \mathcal{V}} \text { has Jordan type } \mu\right\}
$$

Then we have a decomposition $X_{\mu}=\sqcup_{\mu^{\prime} \vdash \operatorname{dim} V-2} X_{\mu, \mu^{\prime}}$ where

$$
X_{\mu, \mu^{\prime}}:=\left\{\mathcal{V} \in X_{\mu}|N|_{(N \mathcal{V})^{\perp} / N \mathcal{V}} \text { has Jordan type } \mu^{\prime}\right\} .
$$

For $\mu^{\prime} \vdash \operatorname{dim} V-2$ we similarly define

$$
X_{\mu^{\prime}}=\left\{l \subset V|\langle l, l\rangle=0, \operatorname{dim} l=1, l \subset \operatorname{ker} N, N|_{l^{\perp} / l} \text { has Jordan type } \mu^{\prime}\right\},
$$

then the natural map $X_{\mu, \mu^{\prime}} \rightarrow X_{\mu^{\prime}}: \mathcal{V} \mapsto N \mathcal{V}$ is a fiber bundle whose fiber at $l \in X_{\mu^{\prime}}$ is $X_{\mu, l}$ defined in Proposition 7.7. Thus we have

$$
\mathcal{X}\left(X_{\mu, \mu^{\prime}}\right)=\mathcal{X}\left(X_{\mu^{\prime}}\right) \mathcal{X}\left(X_{\mu, l_{\mu^{\prime}}}\right), \quad \text { i.e. } \quad \mathcal{X}\left(X_{\mu}\right)=\sum_{\mu^{\prime} \vdash \operatorname{dim} V-2} \mathcal{X}\left(X_{\mu^{\prime}}\right) \mathcal{X}\left(X_{\mu, l_{\mu^{\prime}}}\right)
$$

where $l_{\mu^{\prime}}$ is any element in $X_{\mu^{\prime}}$. Using Proposition 7.3 and 7.7, we get the following result.

Proposition 7.9. For $\mu \vdash \operatorname{dim} V-4$,

if $\mu=\lambda \downarrow_{(i-4)}^{(i)}$ for $i \geq 4$,

if $\mu=\lambda \downarrow_{(i-3, i-3)}^{(i, i-2)}$ for $i \geq 3$,

if $\mu=\lambda \downarrow_{(i-2, i-2)}^{(i, i)}$ for $i \geq 2$,

otherwise,

$$
\begin{aligned}
\mathcal{X}\left(X_{\mu}\right) & =\frac{1-(-1)^{m_{i}}}{2}(-1)^{m_{i-2}} \\
\mathcal{X}\left(X_{\mu}\right) & =\frac{1-(-1)^{m_{i}}}{2}\left(1-(-1)^{m_{i-2}}\right) \\
\mathcal{X}\left(X_{\mu}\right) & =2\left\lfloor\frac{m_{i}}{2}\right\rfloor \\
\mathcal{X}\left(X_{\mu}\right) & =0
\end{aligned}
$$

Combined with Lemma 6.2, this is now a natural consequence. 
Proposition 7.10. Let $\mathfrak{S}_{2} \times W^{\prime} \subset W$ be the maximal parabolic subgroup where $W^{\prime}$ is of the same type as $W$. If $c \in \mathfrak{S}_{2}$ is a 2-cycle, then for $\lambda=\left(1^{m_{1}} 2^{m_{2}} \ldots\right)$ we have

$$
\begin{aligned}
& \operatorname{Res}_{c \cdot W^{\prime}}^{W} \operatorname{ch~TSp}(\lambda)=\sum_{i \geq 2, m_{i} \geq 2} 2\left\lfloor\frac{m_{i}}{2}\right\rfloor \operatorname{ch} \mathbf{T S p}\left(\lambda \downarrow_{(i-2, i-2)}^{(i, i)}\right) \\
& \quad+\sum_{i \geq 4, m_{i} \text { odd }}(-1)^{m_{i-2}} \operatorname{ch~TSp}\left(\lambda \downarrow_{(i-4)}^{(i)}\right)+\sum_{i \geq 3, m_{i}, m_{i-2} \text { odd }} 2 \operatorname{ch~} \mathbf{T S p}\left(\lambda \downarrow_{(i-3, i-3)}^{(i, i-2)}\right) .
\end{aligned}
$$

Here $\mathbf{T S p}\left(\lambda \downarrow_{(i-2, i-2)}^{(i, i)}\right), \mathbf{T S p}\left(\lambda \downarrow_{(i-4)}^{(i)}\right), \mathbf{T S p}\left(\lambda \downarrow_{(i-3, i-3)}^{(i, i-2)}\right)$ are total Springer representations of $W^{\prime}$.

7.3. General case. We consider the general case when $1 \leq k \leq n$. For $\mu \vdash 2 n-2 k$, we define

$$
X_{\mu}=\left\{\mathcal{V} \subset V|\langle\mathcal{V}, \mathcal{V}\rangle=0, \operatorname{dim} \mathcal{V}=k, N \mathcal{V} \subset \mathcal{V}, N|_{\mathcal{V}} \text { is regular, }\left.N\right|_{\mathcal{V}^{\perp} / \mathcal{V}} \text { has Jordan type } \mu\right\}
$$

We again use Proposition 7.3 and 7.7. Let $\mathcal{I}=\left\{\left(\mu^{1}, \mu^{2}, \cdots, \mu^{k-1}\right) \mid \mu^{i} \vdash 2 n-2 i\right\}$. Then there exists a decomposition $X_{\mu}=\bigsqcup_{\left(\mu^{1}, \cdots, \mu^{k-1}\right) \in \mathcal{I}} X_{\mu,\left(\mu^{1}, \cdots, \mu^{k-1}\right)}$ where

$$
X_{\mu,\left(\mu^{1}, \cdots, \mu^{k-1}\right)}=\left\{\mathcal{V} \in X_{\mu}|N|_{\left(N^{k-i} \mathcal{V}\right)^{\perp} /\left(N^{k-i} \mathcal{V}\right)} \text { has Jordan type } \mu^{i} \text { for } 1 \leq i \leq k-1\right\} .
$$

Also there exist natural morphisms

$$
X_{\mu,\left(\mu^{1}, \cdots, \mu^{k-1}\right)} \stackrel{N}{\longrightarrow} X_{\mu^{k-1},\left(\mu^{1}, \cdots, \mu^{k-2}\right)} \stackrel{N}{\rightarrow} \cdots \stackrel{N}{\longrightarrow} X_{\mu^{2},\left(\mu^{1}\right)} \stackrel{N}{\rightarrow} X_{\mu^{1}}
$$

each of which is a fiber bundle with fiber isomorphic to a variety defined in Proposition[7.7 and $X_{\mu^{1}}$ is as in Proposition 7.3 . Now the following proposition is an easy combinatorial exercise.

Proposition 7.11. Let $\mu \vdash 2 n-2 k$. Then,

$$
\begin{array}{ll}
\mu=\lambda \downarrow_{(i-2 k)}^{(i)}, & \mathcal{X}\left(X_{\mu}\right)=\frac{1-(-1)^{m_{i}}}{2}(-1)^{m_{i-2}+m_{i-4}+\cdots+m_{i-2 k+2}} \\
\mu=\lambda \downarrow_{\left(\frac{i+j}{2}-k, \frac{i+j}{2}-k\right)}^{(i, j)} \text { for } i, j \text { s.t. } & \\
i-j \in 2 \mathbb{Z}, 0<\frac{i-j}{2}<k \leq \frac{i+j}{2}, & \mathcal{X}\left(X_{\mu}\right)=\frac{1-(-1)^{m_{i}}}{2}(-1)^{m_{i-2}+m_{i-4}+\cdots+m_{j+2}}\left(1-(-1)^{m_{j}}\right) \\
\mu=\lambda \downarrow_{(i-k, i-k)}^{(i, i)}, & \mathcal{X}\left(X_{\mu}\right)=2\left\lfloor\frac{m_{i}}{2}\right\rfloor \\
\text { otherwise, } & \mathcal{X}\left(X_{\mu}\right)=0 .
\end{array}
$$

For $B$ odd $C$ even $i, j$ such that $i>j, \lambda /\left(\lambda \downarrow_{(j)}^{(i)}\right)$ is a horizontal strip and ht $\left(\lambda /\left(\lambda \downarrow_{(j)}^{(i)}\right)\right) \equiv$ $m_{i-2}+m_{i-4}+\cdots+m_{j+2} \bmod 2$ since $m_{i-1}, m_{i-3}, \cdots, m_{j+3}, m_{j+1}$ are even. Therefore together with Lemma 6.2 we have the following main result of this section. 
Theorem 7.12. Assume $1 \leq k \leq n$. Let $\mathfrak{S}_{k} \times W^{\prime} \subset W$ be the maximal parabolic subgroup where $W^{\prime}$ is of the same type as $W$. If $c \in \mathfrak{S}_{k}$ is a $k$-cycle, then for $\lambda=\left(1^{m_{1}} 2^{m_{2}} \ldots\right)$ we have

$$
\begin{aligned}
\operatorname{Res}_{c \cdot W^{\prime}}^{W} \operatorname{ch} \mathbf{T S p}(\lambda)= & \sum_{i \geq k, m_{i} \geq 2} 2\left\lfloor\frac{m_{i}}{2}\right\rfloor \operatorname{ch} \mathbf{T S p}\left(\lambda \downarrow_{(i-k, i-k)}^{(i, i)}\right) \\
& +\sum_{i \geq 2 k, m_{i} \text { odd }}(-1)^{\operatorname{ht}\left(\lambda /\left(\lambda \downarrow_{(i-2 k)}^{(i)}\right)\right)} \operatorname{ch~} \mathbf{T S p}\left(\lambda \downarrow_{(i-2 k)}^{(i)}\right) \\
& +\sum_{\substack{0<i-j<2 k \leq i+j \\
m_{i}, m_{j} \text { odd }}}(-1)^{\operatorname{ht}\left(\lambda /\left(\lambda \downarrow_{(j)}^{(i)}\right)\right)} 2 \operatorname{ch} \mathbf{T S p}\left(\lambda \downarrow_{\left(\frac{i+j}{2}-k, \frac{i+j}{2}-k\right)}^{(i, j)}\right) .
\end{aligned}
$$

Here $\mathbf{T S p}\left(\lambda \downarrow_{(i-k, i-k)}^{(i, i)}\right), \mathbf{T S p}\left(\lambda \downarrow_{(i-2 k)}^{(i)}\right), \mathbf{T S p}\left(\lambda \downarrow_{\left(\frac{i+j}{2}-k, \frac{i+j}{2}-k\right)}^{(i, j)}\right)$ are total Springer representations of $W^{\prime}$.

\section{Geometric Properties for type $D$}

Here we assume $G=S O_{2 n}(\mathbb{C})$ for $n \geq 2$, acting on $V=\mathbb{C}^{2 n}$ equipped with a nondegenerate symmetric bilinear form $\langle$,$\rangle . Almost every argument in the previous section is still valid in this$ case, but one needs to be careful since

- $\mathcal{B}=G / B$ is no longer the full isotropic flag variety of $V$, and

- for a nilpotent element $N \in \mathfrak{g}$, the Jordan type $\lambda$ of $N$ does not determine $H^{*}\left(\mathcal{B}_{N}\right)$ if $\lambda$ is very even.

Thus we need a minor modification of Theorem 7.12, From now on we state and prove such modifications.

Proposition 8.1. Assume that $k$ is odd and $1 \leq k \leq n-2$. Let $\mathfrak{S}_{k} \times W^{\prime} \subset W$ be the maximal parabolic subgroup where $W^{\prime}$ is of the same type as $W$ which contains both $s_{+}$and $s_{-}$. (See Section 3.3 for the definition of $s_{+}$and $s_{-}$.) If $c \in \mathfrak{S}_{k}$ is a $k$-cycle and $\lambda=\left(1^{m_{1}} 2^{m_{2}} \ldots\right)$, then Theorem 7.12 is still valid except the following cases: if $\lambda$ is very even, then

$$
\operatorname{Res}_{c \cdot W^{\prime}}^{W} \operatorname{ch} \mathbf{T S p}(\lambda+)=\operatorname{Res}_{c \cdot W^{\prime}}^{W} \operatorname{ch} \mathbf{T S p}(\lambda-)=\sum_{i \geq k, m_{i} \geq 2} m_{i} \operatorname{ch} \mathbf{T S p}\left(\lambda \downarrow_{(i-k, i-k)}^{(i, i)}\right) .
$$

Otherwise if $\lambda \downarrow_{(e-k, e-k)}^{(e, e)}$ is very even for some $e \geq k$, then

$$
\begin{aligned}
\operatorname{Res}_{c \cdot W^{\prime}}^{W} \operatorname{ch} \mathbf{T S p}(\lambda)= & \sum_{i \geq k, i \neq e, m_{i} \geq 2} m_{i} \operatorname{ch} \mathbf{T S p}\left(\lambda \downarrow_{(i-k, i-k)}^{(i, i)}\right) \\
& +\operatorname{ch} \mathbf{T S p}\left(\lambda \downarrow_{(e-k, e-k)}^{(e, e)}+\right)+\operatorname{ch} \mathbf{T S p}\left(\lambda \downarrow_{(e-k, e-k)}^{(e, e)}-\right) .
\end{aligned}
$$

Here the total Springer representations on the right hand side are with respect to $W^{\prime}$.

Proof. If there is no very even partition which appears in the formula of Theorem 7.12 then its proof is still valid. Also note that these two cases above are the only ones where a very even partition can appear in the formula for $k$ odd. But the right hand side on the first equation does not contain any very even partition, thus the formula still holds by the same reason. 
Now consider the second case. Then $m_{e}=2$ and $m_{i} \equiv 0 \bmod 2$ for any $i$. Also $\lambda \downarrow_{(i-k, i-k)}^{(i, i)}$ are not very even unless $i=e$. Thus by the same reason as the previous section at least we have

$$
\begin{aligned}
\operatorname{Res}_{c \cdot W^{\prime}}^{W} \operatorname{ch} \mathbf{T S p}(\lambda)= & \sum_{i \geq k, i \neq e, m_{i} \geq 2} m_{i} \operatorname{ch} \mathbf{T S p}\left(\lambda \downarrow_{(i-k, i-k)}^{(i, i)}\right) \\
& +\alpha \operatorname{ch~TSp}\left(\lambda \downarrow_{(e-k, e-k)}^{(e, e)}+\right)+\beta \operatorname{ch} \mathbf{T S p}\left(\lambda \downarrow_{(e-k, e-k)}^{(e, e)}-\right)
\end{aligned}
$$

for some $\alpha, \beta$ such that $\alpha+\beta=\mathcal{X}\left(X_{\lambda \downarrow}^{(e, e)}\left(\begin{array}{l}(e-k, e-k) \\ \text {. }\end{array}\right)=2\right.$. (See Proposition7.11) Recall the involution $\tau$ defined in Section 3.3. Since $|\lambda| \equiv 2 \bmod 4$ in our case, ss-rank $G$ is odd and $\tau$ fixes each conjugacy class in $W$. Thus by applying $\tau$ on both sides we have

$$
\begin{aligned}
\operatorname{Res}_{c \cdot W^{\prime}}^{W} \operatorname{ch} \mathbf{T S p}(\lambda)= & \sum_{i \geq k, i \neq e, m_{i} \geq 2} m_{i} \operatorname{ch} \tau \circ \mathbf{T S p}\left(\lambda \downarrow_{(i-k, i-k)}^{(i, i)}\right) \\
& +\alpha \operatorname{ch} \tau \circ \mathbf{T S p}\left(\lambda \downarrow_{(e-k, e-k)}^{(e, e)}+\right)+\beta \operatorname{ch} \tau \circ \mathbf{T S p}\left(\lambda \downarrow_{(e-k, e-k)}^{(e, e)}-\right) \\
= & \sum_{i \geq k, i \neq e, m_{i} \geq 2} m_{i} \operatorname{ch} \mathbf{T S p}\left(\lambda \downarrow_{(i-k, i-k)}^{(i, i)}\right) \\
& +\alpha \operatorname{ch} \mathbf{T S p}\left(\lambda \downarrow_{(e-k, e-k)}^{(e, e)}-\right)+\beta \operatorname{ch} \mathbf{T S p}\left(\lambda \downarrow_{(e-k, e-k)}^{(e, e)}+\right) .
\end{aligned}
$$

This only happens when $\alpha=\beta=1$ since $\operatorname{ch} \mathbf{T S p}\left(\lambda \downarrow_{(e-k, e-k)}^{(e, e)}+\right)$ and $\operatorname{ch} \mathbf{T S p}\left(\lambda \downarrow_{(e-k, e-k)}^{(e, e)}-\right)$ are linearly independent. Thus the result follows.

Proposition 8.2. Assume that $k$ is even and $1 \leq k \leq n-2$. Let $\mathfrak{S}_{k} \times W^{\prime} \subset W$ be the maximal parabolic subgroup where $W^{\prime}$ is of the same type as $W$ which contains both $s_{+}$and $s_{-}$. (See Section 3.3 for the definition of $s_{+}$and $s_{-}$.) If $c \in \mathfrak{S}_{k}$ is a $k$-cycle and $\lambda=\left(1^{m_{1}} 2^{m_{2}} \ldots\right)$, then Theorem 7.12 is still valid except the following cases: if $\lambda \downarrow \frac{\left(\frac{e_{1}+e_{2}}{2}-k, \frac{e_{1}+e_{2}}{2}-k\right)}{\left(e_{1}, e_{2}\right.}$ is very even for some odd $e_{1}, e_{2}$ such that $0<e_{1}-e_{2}<2 k \leq e_{1}+e_{2}$, then

$$
\begin{aligned}
& \operatorname{Res}_{c \cdot W^{\prime}}^{W} \operatorname{ch} \mathbf{T S p}(\lambda)=\sum_{i \geq k, m_{i} \geq 2} 2\left\lfloor\frac{m_{i}}{2}\right\rfloor \operatorname{ch} \mathbf{T S p}\left(\lambda \downarrow_{(i-k, i-k)}^{(i, i)}\right) \\
& \left.+\sum_{i \in\left\{e_{1}, e_{2}\right\}, i \geq 2 k}(-1)^{\operatorname{ht}\left(\lambda /\left(\lambda \downarrow_{(i-2 k)}^{(i)}\right)\right.}\right) \operatorname{ch} \mathbf{T S p}\left(\lambda \downarrow_{(i-2 k)}^{(i)}\right) \\
& +(-1)^{\operatorname{ht}\left(\lambda /\left(\lambda \downarrow_{\left(e_{2}\right)}^{\left(e_{1}\right)}\right)\right)} \operatorname{ch}\left(\operatorname{TSp}\left(\lambda \downarrow_{\left(\frac{\downarrow_{1}+e_{2}}{2}-k, \frac{e_{1}+e_{2}}{2}-k\right)}^{\left(e_{1}, e_{2}\right)}+\right) \oplus \mathbf{T S p}\left(\lambda \downarrow_{\left(\frac{e_{1}+e_{2}}{2}-k, \frac{e_{1}+e_{2}}{2}-k\right)}^{\left(e_{1}, e_{2}\right)}-\right)\right) .
\end{aligned}
$$

Otherwise if $\lambda$ is very even, then

$$
\begin{aligned}
& \operatorname{Res}_{c \cdot W^{\prime}}^{W} \operatorname{ch} \mathbf{T S p}(\lambda+)=\sum_{i \geq k, m_{i} \geq 2} m_{i} \operatorname{ch} \mathbf{T S p}\left(\lambda \downarrow_{(i-k, i-k)}^{(i, i)}+\right), \\
& \operatorname{Res}_{c \cdot W^{\prime}}^{W} \operatorname{ch} \mathbf{T S p}(\lambda-)=\sum_{i \geq k, m_{i} \geq 2} m_{i} \operatorname{ch} \mathbf{T S p}\left(\lambda \downarrow_{(i-k, i-k)}^{(i, i)}-\right) .
\end{aligned}
$$

Here the total Springer representations on the right hand side are with respect to $W^{\prime}$.

Proof. If there is no very even partition which appears in the formula of Theorem 7.12 then its proof is still valid. Also note that these two cases above are the only ones where a very even 
partition can appear in the formula if $k$ is even. The proof for the first equation is totally analogous of that of the second part in Proposition 8.1.

Now consider the second case for $\mathbf{T S p}(\lambda+)$. (Then the formula for $\mathbf{T S p}(\lambda-)$ can be obtained by applying $\tau$.) Then $m_{i} \equiv 0 \bmod 2$ for any $i$ and $\lambda \downarrow_{(i-k, i-k)}^{(i, i)}$ are all very even for $i \geq k$ if $m_{i} \geq 2$. Thus by the same reason as the previous section at least we have

$$
\operatorname{Res}_{c \cdot W^{\prime}}^{W} \operatorname{ch} \mathbf{T S p}(\lambda+)=\sum_{i \geq k, m_{i} \geq 2}\left(\alpha_{i} \operatorname{ch} \mathbf{T S p}\left(\lambda \downarrow_{(i-k, i-k)}^{(i, i)}+\right)+\beta_{i} \operatorname{ch} \mathbf{T S p}\left(\lambda \downarrow_{(i-k, i-k)}^{(i, i)}-\right)\right)
$$

for some $\alpha_{i}, \beta_{i}$ such that $\alpha_{i}+\beta_{i}=\mathcal{X}\left(X_{\lambda \downarrow_{(i-k, i-k)}^{(i, i)}}\right)=m_{i}$. (See Proposition 7.11) Now for $w=w_{(\rho, \emptyset)-} \in W^{\prime}$ for some even $\rho \vdash n-k$, the argument in Section 3.3 implies that $c w \in W$ is parametrized by $(\rho \cup\{k\}, \emptyset)-$.

Thus if we evaluate the equation at $w=w_{(\rho, \emptyset)-}$ then

$$
0=\operatorname{tr}\left(w_{(\rho \cup\{k\}, \emptyset)-}, \mathbf{T S p}(\lambda+)\right)=\sum_{i \geq k, m_{i} \geq 2} \beta_{i} \operatorname{tr}\left(w_{(\rho, \emptyset)-}, \mathbf{T S p}\left(\lambda \downarrow_{(i-k, i-k)}^{(i, i)}-\right)\right)
$$

by Lemma 3.1. We set

$$
\Xi:=\sum_{i \geq k, m_{i} \geq 2} \beta_{i}\left(\operatorname{ch} \mathbf{T S p}\left(\lambda \downarrow_{(i-k, i-k)}^{(i, i)}+\right)-\operatorname{ch} \mathbf{T S p}\left(\lambda \downarrow_{(i-k, i-k)}^{(i, i)}-\right)\right)
$$

and show $\Xi=0$. As $\operatorname{ch} \mathbf{T S p}\left(\lambda \downarrow_{(i-k, i-k)}^{(i, i)}+\right), \operatorname{ch} \mathbf{T S p}\left(\lambda \downarrow_{(i-k, i-k)}^{(i, i)}-\right)$ for different $i$ are linearly independent, it implies $\beta_{i}=0$, from which the claim follows. Indeed, if $w \in W$ is not of the form $w_{(\rho, \emptyset) \pm}$ for some $\rho \vdash n-k$ even, then obviously $\Xi(w)=0$. If $w=w_{(\rho, \emptyset)-}$ for some even $\rho$, then as $\operatorname{ch} \mathbf{T S p}\left(\lambda \downarrow_{(i-k, i-k)}^{(i, i)}+\right)(w)=0$ for any $i$, we still have $\Xi(w)=0$ from the equation above. Finally if $w=w_{(\rho, \emptyset)+}$ for some even $\rho$, then as $\Xi \circ \tau=-\Xi$, we have $\Xi(w)=(\Xi \circ \tau)(\tau(w))=-\Xi\left(w_{(\rho, \emptyset)-}\right)=0$. It suffices for the proof.

If $k=n-1$, then there is no maximal parabolic subgroup of the form $\mathfrak{S}_{n-1} \times W^{\prime} \subset W$. But we can still calculate the character value of total Springer representations at an $(n-1)$-cycle using the same method; we let $P \subset G$ be a (non-maximal) parabolic subgroup of $G$ such that $W_{P} \subset W$ is a parabolic subgroup of $W$ generated by all the simple reflections except both $s_{+}$and $s_{-}$. Then $\mathcal{P}=G / P$ can be regarded as the Grassmannian of isotropic $(n-1)$-dimensional subspaces in $V$ and there exists a natural projection $\pi_{P}: \mathcal{B} \rightarrow \mathcal{P}$. Also for any $\mathcal{V} \in \mathcal{P}_{N}$ such that $\left.N\right|_{\mathcal{V}}$ is regular, $\pi_{P}^{-1}(\mathcal{V})$ consists of only one point. Therefore we deduce the following proposition.

Proposition 8.3. Let $\mathfrak{S}_{n-1} \subset W$ be a parabolic subgroup of $W$ and $c \in \mathfrak{S}_{n-1}$ be a $(n-1)$-cycle. If $n \geq 3$, then we have

$$
\begin{aligned}
\operatorname{ch} \mathbf{T S p}(\lambda)(c)= & 2 \delta_{\lambda,(n-1, n-1,1,1)}+2 \delta_{\lambda,(n, n)}+\delta_{\lambda,(2 n-1,1)} 2 \delta_{\lambda,(i, j, 1,1)}+\sum_{i>j>1} \sum_{i>j \geq 1 \text { odd }, i+j=2 n} 2 \delta_{\lambda,(i, j)} .
\end{aligned}
$$

This formula holds even when $\lambda$ is very even. If $n=2$, then $c=i d$ and we have

$$
\operatorname{ch} \mathbf{T S p}(\lambda)(i d)=\operatorname{dim} \mathbf{T S p}(\lambda)=4 \delta_{\lambda,(1,1,1,1)}+2 \delta_{\lambda,(2,2)}+\delta_{\lambda,(3,1)} .
$$

This formula holds even when $\lambda$ is very even, i.e. when $\lambda=(2,2)$. 
Proof. The same proof as Theorem 7.12 applies here.

Remark. This theorem can be interpreted in an analogous way to Theorem 7.12 if we decree that $\operatorname{ch} \mathbf{T S p}(1,1)=1$.

If $k=n$, then there are two maximal parabolic subgroups of $W$ isomorphic to $\mathfrak{S}_{n}$, i.e. $\mathfrak{S}_{n \pm}$ defined in Section 3.3 (In fact, if $n=4$ then there are three possible choices. But we discard the choice of the parabolic subgroup containing both $s_{+}$and $s_{-}$.) We define $P_{+}, P_{-} \subset G$ to be two parabolic subgroups whose Weyl groups are $\mathfrak{S}_{n+}, \mathfrak{S}_{n-}$, respectively. Then we may identify $G / P_{+}, G / P_{-}$with two components of the Lagrangian Grassmannian of $V$. Also there exists a natural map $\pi_{+}: \mathcal{B} \rightarrow G / P_{+}, \pi_{-}: \mathcal{B} \rightarrow G / P_{-}$such that for any isotropic flag $\mathcal{F} \in \mathcal{B}, \pi_{+}(\mathcal{F}), \pi_{-}(\mathcal{F})$ are two Lagrangian subspaces containing $\mathcal{F}$.

Suppose $c_{ \pm} \in \mathfrak{S}_{n \pm}$ is a $n$-cycle. If $n=2$, then by direct calculation we have

$$
\begin{gathered}
\operatorname{ch} \mathbf{T S p}((3,1))\left(c_{ \pm}\right)=1, \quad \operatorname{ch} \mathbf{T S p}((2,2) \pm)\left(c_{ \pm}\right)=2 \\
\operatorname{ch} \mathbf{T S p}((2,2) \mp)\left(c_{ \pm}\right)=0, \quad \operatorname{ch} \mathbf{T S p}((1,1,1,1))\left(c_{ \pm}\right)=4 .
\end{gathered}
$$

Now suppose $n \geq 3$. If we follow the argument in the previous section it is required to calculate the Euler characteristic of

$$
X_{ \pm}:=\left\{\mathcal{V} \in G / P_{ \pm}|N \mathcal{V} \subset \mathcal{V}, N|_{\mathcal{V}} \text { is regular }\right\} .
$$

To that end we mimic Section 7.3 let $\mathcal{I}=\left\{\left(\mu^{1}, \mu^{2}, \cdots, \mu^{n-2}\right) \mid \mu^{i} \vdash 2 n-2 i\right\}$. Then there exists a decomposition $X_{ \pm}=\bigsqcup_{\left(\mu^{1}, \cdots, \mu^{n-2}\right) \in \mathcal{I}} X_{ \pm,\left(\mu^{1}, \cdots, \mu^{n-2}\right)}$ where

$$
X_{ \pm,\left(\mu^{1}, \cdots, \mu^{n-2}\right)}:=\left\{\mathcal{V} \in X_{ \pm}|N|_{\left(N^{k-i} \mathcal{V}\right)^{\perp} /\left(N^{k-i} \mathcal{V}\right)} \text { has Jordan type } \mu^{i} \text { for } 1 \leq i \leq n-2\right\} .
$$

Also there exist natural morphisms

$$
X_{ \pm,\left(\mu^{1}, \cdots, \mu^{n-2}\right)} \stackrel{N^{2}}{\longrightarrow} X_{\mu^{n-2},\left(\mu^{1}, \cdots, \mu^{n-3}\right)} \stackrel{N}{\longrightarrow} \cdots \stackrel{N}{\longrightarrow} X_{\mu^{2},\left(\mu^{1}\right)} \stackrel{N}{\longrightarrow} X_{\mu^{1}}
$$

each of which is a fiber bundle with fiber isomorphic to a variety defined in Proposition 7.7 except the first map, and $X_{\mu^{1}}$ is as in Proposition 7.3 .

In order to proceed induction, it remains to calculate the Euler characteristic of a fiber of the first map. To that end, it is enough to assume $G=S O_{6}(\mathbb{C})$ and calculate the Euler characteristic of

$$
X_{ \pm, l}:=\left\{\mathcal{V} \in G / P_{ \pm}|N \mathcal{V} \subset \mathcal{V}, N|_{\mathcal{V}} \text { is regular, } N^{2} \mathcal{V}=l\right\}
$$

for any isotropic line $l \subset \operatorname{ker} N$. We list all the possibilities below.

- $N$ has Jordan type $(5,1),\left.N\right|_{l^{\perp} / l}$ has Jordan type $(3,1): \mathcal{X}\left(X_{ \pm, l}\right)=1$.

- $N$ has Jordan type $(3,3),\left.N\right|_{l^{\perp} / l}$ has Jordan type $(2,2)$ : there are exactly two such isotropic lines and we have $\mathcal{X}\left(X_{+, l}\right)+\mathcal{X}\left(X_{-, l}\right)=1$.

- Otherwise $\mathcal{X}\left(X_{ \pm, l}\right)=0$.

Now we use combinatorial induction to deduce the following. 
Proposition 8.4. Let $\mathfrak{S}_{n \pm} \subset W$ be a maximal parabolic subgroup of $W$ and $c_{ \pm} \in \mathfrak{S}_{n \pm}$ be a n-cycle. If $n$ is even, then

$$
\begin{aligned}
& \operatorname{ch} \mathbf{T S p}((i, j))\left(c_{ \pm}\right)=1 \quad \text { if } i>j \text { are odd and } i+j=2 n, \\
& \operatorname{ch} \mathbf{T S p}((n, n) \pm)\left(c_{ \pm}\right)=2, \quad \operatorname{ch} \mathbf{T S p}((n, n) \mp)\left(c_{ \pm}\right)=0,
\end{aligned}
$$

and $\operatorname{ch} \mathbf{T S p}(\lambda)\left(c_{ \pm}\right)=0$ otherwise. If $n$ is odd, then $c_{+}$and $c_{-}$are conjugate to each other and we have

$$
\operatorname{ch} \mathbf{T S p}((i, j))\left(c_{ \pm}\right)=1 \text { if } i>j \text { are odd and } i+j=2 n, \quad \operatorname{ch} \mathbf{T S p}((n, n))\left(c_{ \pm}\right)=1,
$$

and $\operatorname{ch~} \mathbf{T S p}(\lambda)\left(c_{ \pm}\right)=0$ otherwise.

Proof. We first calculate $\operatorname{ch} \mathbf{T S p}(\lambda)\left(c_{+}\right)+\operatorname{ch} \mathbf{T S p}(\lambda)\left(c_{-}\right)$. By the observation above and using the same method as Section 7.3 , we see that

$$
\operatorname{ch} \mathbf{T S p}(\lambda)\left(c_{+}\right)+\operatorname{ch} \mathbf{T S p}(\lambda)\left(c_{-}\right)=2\left(\delta_{\lambda,(n, n)}+\sum_{i>j \text { odd }, i+j=2 n} \delta_{\lambda,(i, j)}\right)
$$

which holds even when $\lambda$ is very even. Now the theorem follows from two observations: (1) if $\lambda$ is not very even, then $\operatorname{ch} \mathbf{T S p}(\lambda)\left(c_{+}\right)=\operatorname{ch} \mathbf{T S p}(\lambda)\left(c_{-}\right)$, and (2) if $\lambda$ is very even, then $\operatorname{ch} \mathbf{T S p}(\lambda \mp)\left(c_{ \pm}\right)=0$. (cf. Lemma 3.1)

We summarize previous propositions as follows.

Theorem 8.5. Assume $1 \leq k \leq n$. Let $\mathfrak{S}_{k} \times W^{\prime} \subset W$ be a parabolic subgroup where $W^{\prime}$ is of the same type as $W$. If $1 \leq k \leq n-2$, then we choose $W^{\prime}$ such that $\mathfrak{S}_{k} \times W^{\prime}$ becomes a maximal parabolic subgroup of $W .\left(W^{\prime}\right.$ is trivial if $k=n-1, n$.) If $c \in \mathfrak{S}_{k}$ is a $k$-cycle and $\lambda=\left(1^{m_{1}} 2^{m_{2}} \cdots\right)$ then we have

$$
\begin{aligned}
\operatorname{Res}_{c \cdot W^{\prime}}^{W} \operatorname{ch} \widetilde{\mathbf{T S p}}(\lambda)= & \sum_{i \geq k, m_{i} \geq 2} 2\left\lfloor\frac{m_{i}}{2}\right\rfloor \operatorname{ch} \widetilde{\mathbf{T S p}}\left(\lambda \downarrow_{(i-k, i-k)}^{(i, i)}\right) \\
& +\sum_{i \geq 2 k, m_{i} \text { odd }}(-1)^{\operatorname{ht}\left(\lambda /\left(\lambda \downarrow_{(i-2 k)}^{(i)}\right)\right)} \operatorname{ch\widetilde {\mathbf {TSp}}}\left(\lambda \downarrow_{(i-2 k)}^{(i)}\right) \\
& +\sum_{0<i-j<2 k \leq i+j, m_{i}, m_{j} \text { odd }}(-1)^{\operatorname{ht}\left(\lambda /\left(\lambda \downarrow_{(j)}^{(i)}\right)\right)} 2 \operatorname{ch} \widetilde{\mathbf{T S p}}\left(\lambda \downarrow_{\left(\frac{i+j}{2}-k, \frac{i+j}{2}-k\right)}^{(i, j)}\right) .
\end{aligned}
$$

Here $\widetilde{\mathbf{T S p}}\left(\lambda \downarrow_{(i-k, i-k)}^{(i, i)}\right), \widetilde{\mathbf{T S p}}\left(\lambda \downarrow_{(i-2 k)}^{(i)}\right), \widetilde{\mathbf{T S p}}\left(\lambda \downarrow_{\left(\frac{i+j}{2}-k, \frac{i+j}{2}-k\right)}^{(i, j)}\right)$ are with respect to $W^{\prime}$. Also, we use the convention that

$$
\operatorname{ch} \widetilde{\mathbf{T S p}}((1,1))=2 \quad \text { and } \quad \operatorname{ch} \widetilde{\mathbf{T S p}}(\emptyset)=1 .
$$

Proof. This is just a reformulation of Proposition 8.1 8.4,

\section{Symmetric FunCtions}

Here, we review the theory of symmetric functions and how it is related to the representation theory of Weyl groups for type $A$ and $B C$. For more details, we refer readers to [el81, Sta86, Mac95], etc. 
9.1. Symmetric functions and symmetric groups. Let $\Lambda=\Lambda(x)$ be the ring of symmetric functions of infinitely many variables $x=\left(x_{1}, x_{2}, \cdots\right)$ over $\mathbb{Q}$. For any $\mathbb{Q}$-algebra $R$, we write $\Lambda_{R}:=\Lambda \otimes_{\mathbb{Q}} R$. Define $\Lambda^{n}$ to be the homogeneous part of degree $n$ in $\Lambda$ so that $\Lambda=\bigoplus_{n>0} \Lambda^{n}$. There are series of symmetric functions parametrized by partitions, some of which we recall in the following.

- $h_{\lambda}=h_{\lambda}(x)$ : (complete) homogeneous symmetric functions [Sta86, Chapter 7.5].

- $p_{\lambda}=p_{\lambda}(x)$ : power sum symmetric functions [Sta86, Chapter 7.7].

- $s_{\lambda}=s_{\lambda}(x)$ : Schur functions Sta86, Chapter 7.10, 7.15].

Each series forms a $\mathbb{Q}$-basis of $\Lambda$.

There exists a non-degenerate symmetric bilinear form $\langle\rangle:, \Lambda \times \Lambda \rightarrow \mathbb{Q}$ such that $\left\langle p_{\lambda}, p_{\mu}\right\rangle=$ $\delta_{\lambda, \mu} z_{\lambda}$ and $\left\langle s_{\lambda}, s_{\mu}\right\rangle=\delta_{\lambda, \mu}$ (ref. [Sta86, Chapter 7.9]). Also there exists a ring involution $\omega: \Lambda \rightarrow \Lambda$ satisfying $\omega\left(p_{\lambda}\right)=(-1)^{|\lambda|-l(\lambda)} p_{\lambda}$ and $\omega\left(s_{\lambda}\right)=s_{\lambda^{\prime}}$ (ref. [Sta86, Chapter 7.6]). It is obvious that $\omega$ is an isometry with respect to $\langle$,$\rangle .$

Recall the Frobenius characteristic map (ref. [Sta86, Chapter 7.18])

$$
\Psi: \bigoplus_{n \geq 0} \mathcal{R}\left(\mathfrak{S}_{n}\right) \rightarrow \Lambda
$$

which sends $f \in \mathcal{R}\left(\mathfrak{S}_{n}\right)$ to $\frac{1}{n !} \sum_{w \in \mathfrak{S}_{n}} f(w) p_{\rho(w)} \in \Lambda^{n}$, where $\rho(w)$ is the cycle type of $w$. It is a $\mathbb{Q}$-linear isomorphism and also an isometry. Moreover, it satisfies

$$
\Psi\left(\operatorname{Ind}_{\mathfrak{S}_{m} \times \mathfrak{S}_{n}}^{\mathfrak{S}_{m+n}} f \times g\right)=\Psi(f) \Psi(g)
$$

for any $f \in \mathcal{R}\left(\mathfrak{S}_{m}\right)$ and $g \in \mathcal{R}\left(\mathfrak{S}_{n}\right)$, and

- $\Psi^{-1}\left(h_{\lambda}\right)=\operatorname{ch}\left(\operatorname{Ind}_{\mathfrak{S}_{\lambda_{1}} \times \cdots \times \mathfrak{S}_{\lambda_{l(\lambda)}}}^{\mathfrak{S}_{|\lambda|}} \operatorname{Id}_{\mathfrak{S}_{\lambda_{1}} \times \cdots \times \mathfrak{S}_{\lambda_{l(\lambda)}}}\right)$,

- $\Psi^{-1}\left(p_{\lambda}\right)\left(w_{\rho}\right)=\delta_{\lambda, \rho} z_{\lambda}$, and

- $\Psi^{-1}\left(s_{\lambda}\right)=\operatorname{ch} \chi^{\lambda}$.

9.2. Hall-Littlewood functions, Green and Kostka-Foulkes polynomials. Here, we consider $\Lambda_{\mathbb{Q}[t]}$ and recall the Hall-Littlewood functions. (ref. [Mac95, Chapter III.2])

- $P_{\lambda}(t)=P_{\lambda}(x ; t)$ : the Hall-Littlewood $P$-functions

- $Q_{\lambda}(t)=Q_{\lambda}(x ; t)$ : the Hall-Littlewood $Q$-functions

Then $\left\{P_{\lambda}(t)\right\}$ forms a $\mathbb{Q}[t]$-basis of $\Lambda_{\mathbb{Q}[t]}$, but $\left\{Q_{\lambda}(t)\right\}$ does not. (It becomes a basis if we base change $\lambda_{\mathbb{Q}[t]}$ to $\mathbb{Q}(t)$.) There exists a non-degenerate symmetric bilinear form $\langle\langle\rangle\rangle:, \Lambda_{\mathbb{Q}[t]} \times \Lambda_{\mathbb{Q}[t]} \rightarrow \mathbb{Q}(t)$ defined by $\left\langle\left\langle P_{\lambda}(t), Q_{\mu}(t)\right\rangle\right\rangle=\delta_{\lambda, \mu}$ and $\left\langle\left\langle p_{\lambda}, p_{\mu}\right\rangle\right\rangle=z_{\lambda}(t) \delta_{\lambda, \mu}$ where

$$
z_{\lambda}(t):=\prod_{i \geq 1} \frac{i^{m_{i}} m_{i} !}{\left(1-t^{i}\right)^{m_{i}}} \text { for } \lambda=\left(1^{m_{1}} 2^{m_{2}} \cdots\right)
$$

As $z_{\lambda}(0)=z_{\lambda}$, the former scalar product $\langle$,$\rangle can be regarded as \left\langle\langle,\rangle_{t=0}\right.$.

There is an endomorphism $\mho: \Lambda_{\mathbb{Q}(t)} \rightarrow \Lambda_{\mathbb{Q}(t)}$ which maps $f(x)$ to $f(x /(1-t))$ in Lambda-ring notation, i.e. which substitutes variables $\left\{x_{i} \mid i \geq 1\right\}$ with $\left\{x_{i} t^{j} \mid i \geq 1, j \geq 0\right\}$. In other words, $\mho$ 
is characterized by $\mho(t)=t$ and $\mho\left(p_{k}\right)=\frac{1}{1-t^{k}} p_{k}$. We define $Q_{\lambda}^{\prime}(t)=Q_{\lambda}^{\prime}(x ; t):=\mho\left(Q_{\lambda}(t)\right)$, called the modified Hall-Littlewood $Q^{\prime}$-functions (ref. Mac95, pp. 234-236]). Then in fact $Q_{\lambda}^{\prime}(t) \in \mathbb{Q}[t]$ and $\left\langle P_{\lambda}(t), Q_{\mu}^{\prime}(t)\right\rangle=\delta_{\lambda, \mu}$, i.e. $\left\{P_{\lambda}(t)\right\}$ and $\left\{Q_{\lambda}^{\prime}(t)\right\}$ are dual $\mathbb{Q}[t]$-bases of $\Lambda_{\mathbb{Q}[t]}$ with respect to the scalar product $\langle$,$\rangle .$

The function $Q_{\lambda}^{\prime}(t)$ enjoys the following properties. For partitions $\lambda$ and $\rho$, we define a polynomial $X_{\mu}^{\lambda}(t) \in \mathbb{Q}[t]$ to be the "transition matrix" between bases $\left\{p_{\rho}\right\}$ and $\left\{P_{\lambda}(t)\right\}$, i.e. such that $p_{\rho}=\sum_{\lambda} X_{\rho}^{\lambda}(t) P_{\lambda}(t)$. Then we have $\left\langle p_{\rho}, Q_{\lambda}^{\prime}(t)\right\rangle=X_{\rho}^{\lambda}(t)$, i.e. $Q_{\lambda}^{\prime}(t)=\sum_{\rho} z_{\rho}^{-1} X_{\rho}^{\lambda}(t) p_{\rho}$. If we define $\mathcal{Q}_{\rho}^{\lambda}(t):=t^{b(\lambda)} X_{\rho}^{\lambda}\left(t^{-1}\right)$, then indeed $\mathcal{Q}_{\rho}^{\lambda}(t) \in \mathbb{Q}[t]$, which we call the Green polynomial originally defined in Gre55. Now the equation above reads

$$
Q_{\lambda}^{\prime}(t)=t^{b(\lambda)} \sum_{\rho} z_{\rho}^{-1} \mathcal{Q}_{\rho}^{\lambda}\left(t^{-1}\right) p_{\rho}
$$

On the other hand, if we write $K_{\mu, \lambda}(t)$ to be the Kostka-Foulkes polynomial, then we have $s_{\mu}=$ $\sum_{\lambda} K_{\mu, \lambda}(t) P_{\lambda}(t)$ and thus $\left\langle s_{\mu}, Q_{\lambda}^{\prime}(t)\right\rangle=K_{\mu, \lambda}(t)$, i.e.

$$
Q_{\lambda}^{\prime}(t)=\sum_{\mu} K_{\mu, \lambda}(t) s_{\mu}
$$

9.3. Product, coproduct, Frobenius and Verschiebung operator. Let $y=\left(y_{1}, y_{2}, \cdots\right)$ be another set of indeterminates. Define the coproduct map $\Delta: \Lambda \rightarrow \Lambda(x) \otimes \Lambda(y): f \mapsto f(x, y)$ which substitutes $x$ with $x \sqcup y$. Also define the product map $\nabla: \Lambda(x) \otimes \Lambda(y) \rightarrow \Lambda$ which sends $f(x) g(y)$ to $f g=f(x) g(x)$. Note that there is a naturally induced scalar product $\langle$,$\rangle on \Lambda(x) \otimes \Lambda(y)$ defined by $\langle a(x) b(y), c(x) d(y)\rangle=\langle a, c\rangle\langle b, d\rangle$. Then we have an adjunction $\langle\Delta(f), g\rangle=\langle f, \nabla(g)\rangle$. A similar results also holds if we consider $\Delta: \Lambda_{\mathbb{Q}[t]} \rightarrow \Lambda(x)_{\mathbb{Q}[t]} \otimes \Lambda(y)_{\mathbb{Q}[t]}, \nabla: \Lambda(x)_{\mathbb{Q}[t]} \otimes \Lambda(y)_{\mathbb{Q}[t]} \rightarrow \Lambda_{\mathbb{Q}[t]}$ and the scalar product $\langle\langle$,$\rangle instead.$

We call $\Lambda \rightarrow \Lambda: f \mapsto f\left[p_{2}\right]$ the (second) Frobenius operator where $f[g]$ denotes the plethysm, that is $f\left[p_{2}\right]$ is obtained by substituting $\left\{x_{1}, x_{2}, \cdots\right\}$ with $\left\{x_{1}^{2}, x_{2}^{2}, \cdots\right\}$. Also we define the (second) Verschiebung operator $\mathbb{V}_{2}: \Lambda \rightarrow \Lambda$ which is characterized by $\mathbb{V}_{2}\left(h_{2 n}\right)=h_{n}$ and $\mathbb{V}_{2}\left(h_{2 n+1}\right)=0$. Then there is an adjunction $\left\langle f\left[p_{2}\right], g\right\rangle=\left\langle f, \mathbb{V}_{2}(g)\right\rangle$ for $f, g \in \Lambda$.

9.4. Hyperoctahedral groups. Suppose $W_{n}$ is the Weyl group of type $B C_{n}$, i.e. $W_{n}=\mathfrak{S}_{n} \ltimes(\mathbb{Z} / 2)^{n}$. Then similar to symmetric groups there exists the Frobenius characteristic map

$$
\Psi: \bigoplus_{n \geq 0} \mathcal{R}\left(W_{n}\right) \rightarrow \Lambda(x) \otimes \Lambda(y)
$$

defined as follows. For partitions $\alpha$ and $\beta$ we define

$$
p_{(\alpha, \beta)}=p_{(\alpha, \beta)}(x, y):=\left(\prod_{i \geq 1}\left(p_{\alpha_{i}}(x)+p_{\alpha_{i}}(y)\right)\right)\left(\prod_{j \geq 1}\left(p_{\beta_{j}}(x)-p_{\beta_{j}}(y)\right)\right) .
$$

Then $f \in \mathcal{R}\left(W_{n}\right)$ is mapped under $\Psi$ to $\frac{1}{n ! 2^{n}} \sum_{w \in W_{n}} f(w) p_{\rho(w)}$, where $\rho(w)$ is the bipartition which parametrizes the conjugacy class of $w \in W_{n}$ (see Section [3). It is a $\mathbb{Q}$-linear isomorphism and also an isometry. Moreover, it satisfies

$$
\Psi\left(\operatorname{Ind}_{W_{m} \times W_{n}}^{W_{m+n}} f \times g\right)=\Psi(f) \Psi(g)
$$

for any $f \in \mathcal{R}\left(W_{m}\right)$ and $g \in \mathcal{R}\left(W_{n}\right)$, and 
- $\Psi^{-1}\left(h_{\lambda}(x)\right)=\operatorname{ch}\left(\operatorname{Ind}_{W_{\lambda_{1}} \times \cdots \times W_{\lambda_{l(\lambda)}}}^{W_{|\lambda|}} \operatorname{Id}_{W_{\lambda_{1}} \times \cdots \times W_{\lambda_{l(\lambda)}}}\right)$,

- $\Psi^{-1}\left(h_{\lambda}(y)\right)=\operatorname{ch}\left(\operatorname{Ind}_{W_{\lambda_{1}} \times \cdots \times W_{\lambda_{l(\lambda)}}}^{W_{|\lambda|}} \widetilde{\operatorname{Sign}}_{W_{\lambda_{1}} \times \cdots \times W_{\lambda_{l(\lambda)}}}\right)$, where $\widetilde{\operatorname{Sign}}_{W_{\lambda_{1}} \times \cdots \times W_{\lambda_{l(\lambda)}}}$ is the tensor product of $\operatorname{sgn}_{W_{\lambda_{1}} \times \cdots \times W_{\lambda_{l(\lambda)}}}$ and the pullback of $\operatorname{sgn}_{\mathfrak{S}_{\lambda_{1}} \times \cdots \times \mathfrak{S}_{\lambda_{l(\lambda)}}}$ under the canonical surjection $W_{\lambda_{1}} \times \cdots \times W_{\lambda_{l(\lambda)}} \rightarrow \mathfrak{S}_{\lambda_{1}} \times \cdots \times \mathfrak{S}_{\lambda_{l(\lambda)}}$,

- $\Psi^{-1}\left(p_{(\alpha, \beta)}\right)\left(w_{(\rho, \sigma)}\right)=\delta_{\alpha, \rho} \delta_{\beta, \sigma} z_{\alpha} z_{\beta} 2^{l(\alpha)+l(\beta)}$, and

- $\Psi^{-1}\left(s_{\alpha}(x) s_{\beta}(y)\right)=\operatorname{ch} \chi^{(\alpha, \beta)}$.

We often identify $\mathfrak{S}_{n} \ltimes\{i d\} \subset W_{n}$ with $\mathfrak{S}_{n}$. Then for $\varrho \in \mathcal{R}\left(\mathfrak{S}_{n}\right)$ and $\varphi \in \mathcal{R}\left(W_{n}\right)$ we have (ref. Zel81, 7.10])

$$
\Psi\left(\operatorname{Ind}_{\mathfrak{S}_{n}}^{W_{n}} \varrho\right)=\Delta(\Psi(\varrho)), \quad \Psi\left(\operatorname{Res}_{\mathfrak{S}_{n}}^{W_{n}} \varphi\right)=\nabla(\Psi(\varphi)) .
$$

Thus in particular, $\Psi\left(\operatorname{Ind}_{\mathfrak{S}_{n}}^{W_{n}} \operatorname{ch~Id}_{\mathfrak{S}_{n}}\right)=\Delta\left(h_{n}\right)=\sum_{i=0}^{n} h_{i}(x) h_{n-i}(y)$ and $\Psi\left(\operatorname{Res}_{\mathfrak{S}_{n}}^{W_{n}} \operatorname{ch} \chi^{(\alpha, \beta)}\right)=$ $\nabla\left(s_{\alpha}(x) s_{\beta}(y)\right)=s_{\alpha} s_{\beta}$.

\section{Green and KostKa-Foulkes polynomials}

Our goal in this section is to prove Theorem 10.5 and 10.6 regarding Green and Kostka-Foulkes polynomials. Let $p_{k}^{*,\langle,\rangle\rangle}: \Lambda_{\mathbb{Q}(t)} \rightarrow \Lambda_{\mathbb{Q}(t)}$ be the operator satisfying $\left\langle\left\langle p_{k}^{*,\langle\langle,\rangle} f, g\right\rangle\right\rangle=\left\langle\left\langle f, p_{k} g\right\rangle\right\rangle$, that is the adjoint to multiplication by $p_{k}$ with respect to $\langle\langle$,$\rangle . It is indeed easy to check that$ $p_{k}^{*,\langle l,\rangle}=\frac{k}{1-t^{k}} \frac{\partial}{\partial p_{k}}$ if we identify $\Lambda_{\mathbb{Q}(t)}$ with $\mathbb{Q}(t)\left[p_{1}, p_{2}, \cdots\right]$.

For an indeterminate $u$ we define

$$
\mathbf{Q}(u):=\prod_{i \geq 1} \frac{1-x_{i} t u}{1-x_{i} u}, \quad \mathbf{F}(u):=\frac{1-u}{1-t u}
$$

as elements in $\Lambda_{\mathbb{Q}[t]}[[u]]$. Then by [Mac95, Chapter III.2, $\left.(2.15)\right], Q_{\lambda}(t)$ is the coefficient of $u_{1}^{\lambda_{1}} u_{2}^{\lambda_{2}} \ldots$ in the expansion of $\prod_{i \geq 1} \mathbf{Q}\left(u_{i}\right) \prod_{i<j} \mathbf{F}\left(u_{i}^{-1} u_{j}\right)$. Even when $\lambda$ is a finite sequence of integers which is not necessarily a partition, we may define $Q_{\lambda}(t)$ as a coefficient of $u_{1}^{\lambda_{1}} u_{2}^{\lambda_{2}} \cdots$ in its expansion.

Now we claim (cf. [Mac95, p.237])

$$
\mathbf{Q}(u)=\exp \left(\sum_{n \geq 1} \frac{1-t^{n}}{n} p_{n} u^{n}\right) \text {, i.e. } \log \mathbf{Q}(u)=\sum_{n \geq 1} \frac{1-t^{n}}{n} p_{n} u^{n}
$$

as elements in $\Lambda_{\mathbb{Q}(t)}[[u]]$. But it is clear since

$$
\begin{aligned}
\log \mathbf{Q}(u) & =\sum_{i \geq 1}\left(\log \left(1-x_{i} t u\right)-\log \left(1-x_{i} u\right)\right)=\sum_{i \geq 1}\left(\sum_{j \geq 1} \frac{\left(x_{i} u\right)^{j}}{j}-\frac{\left(x_{i} t u\right)^{j}}{j}\right) \\
& =\sum_{i \geq 1} \sum_{j \geq 1} \frac{\left(x_{i} u\right)^{j}}{j}\left(1-t^{j}\right)=\sum_{j \geq 1} \frac{p_{i} u^{j}}{j}\left(1-t^{j}\right) .
\end{aligned}
$$


Thus we have $p_{k}^{*,\langle\langle,\rangle} \mathbf{Q}(u)=u^{n} \mathbf{Q}(u)$ and it follows that

$$
p_{k}^{*,\langle\langle,\rangle} \prod_{i \geq 1} \mathbf{Q}\left(u_{i}\right) \prod_{i<j} \mathbf{F}\left(u_{i}^{-1} u_{j}\right)=p_{k}\left(u_{1}, u_{2}, \cdots\right) \prod_{i \geq 1} \mathbf{Q}\left(u_{i}\right) \prod_{i<j} \mathbf{F}\left(u_{i}^{-1} u_{j}\right),
$$

where $p_{k}\left(u_{1}, u_{2}, \cdots\right)$ is the power symmetric function of variables $u_{1}, u_{2}, \cdots$. Therefore,

$$
p_{k}^{*,\langle\langle,\rangle} Q_{\lambda}(t)=\sum_{i \geq 1} Q_{\lambda-k e_{i}}(t) .
$$

Here, we regard $\lambda=\left(\lambda_{1}, \lambda_{2}, \cdots\right)$ as an element of $\bigoplus_{i \geq 1} \mathbb{Z}$ and let $e_{i} \in \bigoplus_{i \geq 1} \mathbb{Z}$ be an element whose $k$-th coordinate is 1 and 0 elsewhere. (Note that $\lambda-k e_{i}$ is not necessarily a partition. Also the right side of the identity is a finite sum since $Q_{\lambda-k e_{i}}(t)=0$ when $i>l(\lambda)$.)

There is a way to inductively define $Q_{\lambda}(t)$ for any finite integer sequence $\lambda \in \bigoplus_{i \geq 1} \mathbb{Z}$ if it is known for any partition. According to [Mac95, p.214], for any $s<r$ we have

$$
\begin{aligned}
& \text { if } r-s \text { is odd, } \quad Q_{(s, r)}(t)=t Q_{(r, s)}(t)+\sum_{i=1}^{\frac{r-s-1}{2}}\left(t^{i+1}-t^{i-1}\right) Q_{(r-i, s+i)}(t), \text { and } \\
& \text { if } r-s \text { is even, } \quad Q_{(s, r)}(t)=t Q_{(r, s)}(t)+\sum_{i=1}^{\frac{r-s-2}{2}}\left(t^{i+1}-t^{i-1}\right) Q_{(r-i, s+i)}(t) \\
& +\left(t^{\frac{r-s}{2}}-t^{\frac{r-s-2}{2}}\right) Q_{\left(\frac{r-s}{2}, \frac{r-s}{2}\right)}(t) .
\end{aligned}
$$

A similar formula holds for any finite sequence of integers. From this, the following lemma can be easily verified. From now on, we write $\mathbb{Q}[t] \cdot Q$ to denote some $\mathbb{Q}[t]$-linear combination of the Hall-Littlewood $Q$-functions that we do not specify.

Lemma 10.1. Let $\lambda$ be a partition and $k \geq 1$.

(1) Let $\lambda=\left(r^{m_{r}}\right)$ for some $m_{r} \geq 0$. Then

$$
\begin{aligned}
& m_{r} \text { even, } \quad \sum_{i=1}^{m_{r}} Q_{\lambda-2 k e_{i}}(t)=(-1)^{k} m_{r} Q_{\lambda \downarrow_{(r-k, r-k)}^{(r, r)}}(t)+(t+1) \mathbb{Q}[t] \cdot Q \\
& m_{r} \text { odd, } \quad \sum_{i=1}^{m_{r}} Q_{\lambda-2 k e_{i}}(t)=(-1)^{k}\left(m_{r}-1\right) Q_{\lambda \downarrow_{(r-k, r-k)}^{(r, r)}}(t)+Q_{\lambda \downarrow_{(r-2 k)}^{(r)}}(t)+(t+1) \mathbb{Q}[t] \cdot Q
\end{aligned}
$$

(2) Let $\lambda=\left(r^{m_{r}+1}\right)$ for some $m_{r} \geq 0$. Then,

$$
\begin{array}{ll}
m_{r} \text { even, } & Q_{\lambda-2 k e_{1}}(t)=Q_{\lambda \downarrow_{(r-2 k)}^{(r)}}(t)+(t+1) \mathbb{Q}[t] \cdot Q \\
& Q_{\lambda-(2 k-1) e_{1}}(t)=Q_{\lambda \downarrow_{(r-2 k+1)}^{(r)}}(t)+(t+1) \mathbb{Q}[t] \cdot Q \\
m_{r} \text { odd, } & Q_{\lambda-2 k e_{1}}(t)=(-1)^{k} 2 Q_{\lambda \downarrow_{(r-k, r-k)}^{(r, r)}}(t)-Q_{\lambda \downarrow_{(r-2 k)}^{(r)}}(t)+(t+1) \mathbb{Q}[t] \cdot Q \\
& Q_{\lambda-(2 k-1) e_{1}}(t)=-Q_{\lambda \downarrow_{(r-2 k+1)}^{(r)}}(t)+(t+1) \mathbb{Q}[t] \cdot Q
\end{array}
$$


(3) Let $\lambda=\left(r^{m_{r}+2}\right)$ for some $m_{r} \geq 0$. Then,

$$
Q_{\lambda-k\left(e_{1}+e_{2}\right)}(t)=Q_{\lambda \downarrow_{(r-k, r-k)}^{(r, r)}}(t)+(t+1) \mathbb{Q}[t] \cdot Q
$$

By applying the above lemma repeatedly, for any partition $\lambda$ we easily obtain

$$
\begin{aligned}
& p_{2 k}^{*,\langle\langle,\rangle} Q_{\lambda}(t)=\sum_{i \geq 1} Q_{\lambda-2 k e_{i}}(t) \\
= & \left.\sum_{i \geq k, m_{i} \geq 2}(-1)^{k} 2\left\lfloor\frac{m_{i}}{2}\right\rfloor Q_{\left.\lambda \downarrow_{(i-k, i-k)}^{(i, i)}\right)}(t)+\sum_{i \geq 2 k, m_{i} \text { odd }}(-1)^{\mathrm{ht}\left(\lambda / \lambda \downarrow_{(i-2 k)}^{(i)}\right)}\right)_{Q_{\lambda \downarrow_{(i-2 k)}^{(i)}}(t)}(t) \\
& +\sum_{\substack{i-j \in 2 \mathbb{Z}, 0<i-j<2 k \leq i+j, m_{i}, m_{j} \text { odd }}}(-1)^{\mathrm{ht}\left(\lambda / \lambda \downarrow_{(j)}^{(i)}\right)}(-1)^{\frac{2 k-(i-j)}{2}} 2 Q_{\lambda \downarrow_{\left(\frac{i+j}{2}-k, \frac{i+j}{2}-k\right)}^{(i, j)}}(t) \\
& +(t+1) \mathbb{Q}[t] \cdot Q .
\end{aligned}
$$

We need the following lemma to simplify the signs that appear in the equation above.

Lemma 10.2. Let $\lambda$ be a partition and $\lambda^{\prime}=\left(\lambda_{1}^{\prime}, \lambda_{2}^{\prime}, \cdots\right)$ be its conjugate. Also assume $k \geq 1$.

(1) For $i \geq k, b(\lambda) \equiv b\left(\lambda \downarrow_{(i-k)}^{(i)}\right)+\sum_{a=i-k+1}^{i} \lambda_{a}^{\prime}+k \bmod 2$.

(2) For $i \geq k, b(\lambda) \equiv b\left(\lambda \downarrow_{(i-k, i-k)}^{(i, i)}\right)+k \bmod 2$.

(3) For $0<i-j<2 k \leq i+j$ such that $i-j \in 2 \mathbb{Z}_{>0}$,

$$
b(\lambda) \equiv b\left(\lambda \downarrow_{\left(\frac{i+j}{2}-k, \frac{i+j}{2}-k\right)}^{(i, j)}\right)+\sum_{a=j+1}^{i} \lambda_{a}^{\prime}+\frac{2 k-(i-j)}{2} \bmod 2 .
$$

Proof. Since $\left(\begin{array}{l}n \\ 2\end{array}\right)-\left(\begin{array}{c}n-1 \\ 2\end{array}\right)=n-1$, we have

$$
b(\lambda)-b\left(\lambda \downarrow_{(i-k)}^{(i)}\right)=\sum_{a=i-k+1}^{i}\left(\begin{array}{c}
\lambda_{a}^{\prime} \\
2
\end{array}\right)-\left(\begin{array}{c}
\lambda_{a}^{\prime}-1 \\
2
\end{array}\right)=\sum_{a=i-k+1}^{i}\left(\lambda_{a}^{\prime}-1\right) \equiv \sum_{a=i-k+1}^{i} \lambda_{a}^{\prime}+k \bmod 2 .
$$

Now the rest follows directly from (1). 
Therefore, we have

$$
\begin{aligned}
& p_{2 k}^{*,\langle l,\rangle} \varepsilon_{\lambda} Q_{\lambda}(t)=\varepsilon_{\lambda} \sum_{i \geq 1} Q_{\lambda-2 k e_{i}}(t) \\
= & \sum_{i \geq k, m_{i} \geq 2} \varepsilon_{\lambda \downarrow_{(i-k, i-k)}^{(i, i)}} 2\left\lfloor\frac{m_{i}}{2}\right\rfloor Q_{\lambda \downarrow_{(i-k, i-k)}^{(i, i)}}(t) \\
& +\sum_{i \geq 2 k, m_{i} \text { odd }} \varepsilon_{\lambda \downarrow_{(i-2 k)}^{(i)}}(-1)^{\mathrm{ht}\left(\lambda / \lambda \downarrow_{(i-2 k)}^{(i)}\right)+\sum_{a=i-2 k+1}^{i} \lambda_{a}^{\prime}} Q_{\lambda \downarrow_{(i-2 k)}^{(i)}}(t) \\
& +\sum_{i-j \in 2 \mathbb{Z}, 0<i-j<2 k \leq i+j,}^{m_{i}, m_{j} \text { odd }} \varepsilon_{\lambda \downarrow_{\left(\frac{i+j}{2}-k, \frac{i+j}{2}-k\right)}^{(i, j)}}(-1)^{\mathrm{ht}\left(\lambda / \lambda \downarrow_{(j)}^{(i)}\right)+\sum_{a=j+1}^{i} \lambda_{a}^{\prime}} 2 Q_{\lambda \downarrow_{\left(\frac{i+j}{2}-k, \frac{i+j}{2}-k\right)}^{(i, j)}}(t) \\
& +(t+1) \mathbb{Q}[t] \cdot Q .
\end{aligned}
$$

We apply $\mho$ on both sides (see Section 9 for the definition of $\mho$ ). As mentioned previously, the Hall-Littlewood $Q$-functions become the modified Hall-Littlewood $Q^{\prime}$-functions. We define $p_{k}^{*,\langle,\rangle}$ to be the adjoint operator to the multiplication by $p_{k}$ with respect to $\langle$,$\rangle . Then it is easy to see that$ $p_{k}^{*,\langle,\rangle}=k \frac{\partial}{\partial p_{k}}$ and we have $\mho \circ p_{k}^{*,\langle\langle,\rangle\rangle}=p_{k}^{*,\langle,\rangle} \circ \mho$. (It easily follows from the chain rule.) Therefore, we have

$$
\begin{aligned}
& p_{2 k}^{*,\langle,\rangle} \varepsilon_{\lambda} Q_{\lambda}^{\prime}(t) \\
& =\sum_{i \geq k, m_{i} \geq 2} \varepsilon_{\lambda \downarrow_{(i-k, i-k)}^{(i, i)}} 2\left\lfloor\frac{m_{i}}{2}\right\rfloor Q_{\lambda \downarrow_{(i-k, i-k)}^{(i, i)}}^{\prime}(t) \\
& +\sum_{i \geq 2 k, m_{i} \text { odd }} \varepsilon_{\lambda \downarrow_{(i-2 k)}^{(i)}}(-1)^{\mathrm{ht}\left(\lambda / \lambda \downarrow_{(i-2 k)}^{(i)}\right)+\sum_{a=i-2 k+1}^{i} \lambda_{a}^{\prime}} Q_{\lambda \downarrow_{(i-2 k)}^{(i)}}^{\prime}(t)
\end{aligned}
$$

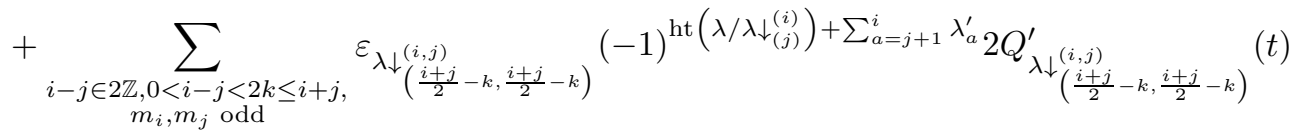

$$
\begin{aligned}
& +(t+1) \mathbb{Q}[t] \cdot Q^{\prime}
\end{aligned}
$$

where $\mathbb{Q}[t] \cdot Q^{\prime}$ is some $\mathbb{Q}[t]$-linear combination of $Q^{\prime}$-functions. Note that the equation above is defined in $\Lambda_{\mathbb{Q}[t]}$. Thus we can evaluate both sides at $t=-1$, which gives

$$
\begin{aligned}
& p_{2 k}^{*,\langle,\rangle} \varepsilon_{\lambda} Q_{\lambda}^{\prime}(-1) \\
= & \sum_{i \geq k, m_{i} \geq 2} \varepsilon_{\lambda \downarrow_{(i-k, i-k)}^{(i, i)}} 2\left\lfloor\frac{m_{i}}{2}\right\rfloor Q_{\lambda \downarrow_{(i-k, i-k)}^{(i, i)}}^{\prime}(-1) \\
& +\sum_{i \geq 2 k, m_{i} \text { odd }} \varepsilon_{\left.\lambda \downarrow_{(i-2 k)}^{(i)}\right)}(-1)^{\mathrm{ht}\left(\lambda / \lambda \downarrow_{(i-2 k)}^{(i)}\right)+\sum_{a=i-2 k+1}^{i} \lambda_{a}^{\prime}} Q_{\lambda_{(i-2 k)}^{(i)}}^{\prime(i)}(-1) \\
& +\sum_{\substack{i-j \in 2 \mathbb{Z}, 0<i-j<2 k \leq i+j, m_{i}, m_{j} \text { odd }}} \varepsilon_{\lambda \downarrow_{\left(\frac{i+j}{2}-k, \frac{i+j}{2}-k\right)}^{(i, j)}}(-1)^{\mathrm{ht}\left(\lambda / \lambda \downarrow_{(j)}^{(i)}\right)+\sum_{a=j+1}^{i} \lambda_{a}^{\prime}} 2 Q_{\lambda \downarrow_{\left(\frac{i+j}{2}-k, \frac{i+j}{2}-k\right)}^{(i, j)}}^{\prime}(-1) .
\end{aligned}
$$

If we take the scalar product $\langle$,$\rangle on both sides with p_{\rho}$ for some $\rho \vdash|\lambda|-2 k$, then the following proposition is a direct outcome. 
Proposition 10.3. For a partition $\lambda=\left(1^{m_{1}} 2^{m_{2}} \ldots\right)$ with its transpose $\lambda^{\prime}=\left(\lambda_{1}^{\prime}, \lambda_{2}^{\prime}, \cdots\right)$ and $k \geq 1$, we have

$$
\begin{aligned}
\mathcal{Q}_{\rho \cup(2 k)}^{\lambda}(-1)= & \sum_{i \geq k, m_{i} \geq 2} 2\left\lfloor\frac{m_{i}}{2}\right\rfloor \mathcal{Q}_{\rho}^{\lambda \downarrow_{(i-k, i-k)}^{(i, i)}}(-1) \\
& +\sum_{i \geq 2 k, m_{i} \text { odd }}(-1)^{\mathrm{ht}\left(\lambda / \lambda \downarrow_{(i-2 k)}^{(i)}\right)+\sum_{a=i-2 k+1}^{i} \lambda_{a}^{\prime}} \mathcal{Q}_{\rho}^{\lambda \downarrow_{(i-2 k)}^{(i)}}(-1) \\
& +\sum_{\substack{i-j \in 2 \mathbb{Z}, 0<i-j<2 k \leq i+j, m_{i}, m_{j} \text { odd }}}(-1)^{\mathrm{ht}\left(\lambda / \lambda \downarrow_{(j)}^{(i)}\right)+\sum_{a=j+1}^{i} \lambda_{a}^{\prime}} 2 \mathcal{Q}_{\rho}^{\lambda \downarrow_{(i+j) / 2-k,(i+j) / 2-k)}^{(i, j)}}(-1) .
\end{aligned}
$$

On the other hand, if we take the scalar product $\langle$,$\rangle on both sides of (10.1) with s_{\nu}$ for some $\nu \vdash|\lambda|-2 k$, then

$$
\begin{aligned}
& \varepsilon_{\lambda}\left\langle Q_{\lambda}^{\prime}(-1), p_{2 k} s_{\nu}\right\rangle \\
& =\sum_{i \geq k, m_{i} \geq 2} \varepsilon_{\lambda \downarrow_{(i-k, i-k)}^{(i, i)}} 2\left\lfloor\frac{m_{i}}{2}\right\rfloor K_{\nu, \lambda \downarrow_{(i-k, i-k)}^{(i, i)}}(-1) \\
& +\sum_{i \geq 2 k, m_{i} \text { odd }} \varepsilon_{\lambda \downarrow_{(i-2 k)}^{(i)}}(-1)^{\mathrm{ht}\left(\lambda / \lambda \downarrow_{(i-2 k)}^{(i)}\right)+\sum_{a=i-2 k+1}^{i} \lambda_{a}^{\prime}} K_{\nu, \lambda \downarrow_{(i-2 k)}^{(i)}}(-1)
\end{aligned}
$$

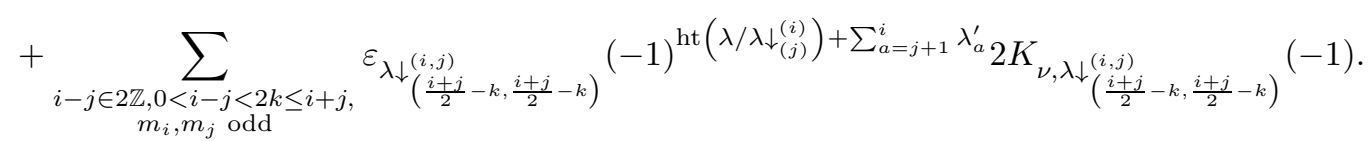

By the Murnaghan-Nakayama rule (cf. [Sta86, 7.17]), we have

Proposition 10.4. For a partition $\lambda=\left(1^{m_{1}} 2^{m_{2}} \ldots\right)$ with its transpose $\lambda^{\prime}=\left(\lambda_{1}^{\prime}, \lambda_{2}^{\prime}, \cdots\right)$ and $k \geq 1$, we have

$$
\begin{aligned}
& \varepsilon_{\lambda} \sum_{\mu \vdash n, \mu / \nu \text { border strip }}(-1)^{\mathrm{ht}(\mu / \nu)} K_{\mu, \lambda}(-1) \\
& =\sum_{i \geq k, m_{i} \geq 2} \varepsilon_{\lambda \downarrow_{(i-k, i-k)}^{(i, i)}} 2\left\lfloor\frac{m_{i}}{2}\right\rfloor K_{\nu, \lambda \downarrow_{(i-k, i-k)}^{(i, i)}}(-1) \\
& +\sum_{i \geq 2 k, m_{i} \text { odd }} \varepsilon_{\lambda \downarrow_{(i-2 k)}^{(i)}}(-1)^{\mathrm{ht}\left(\lambda / \lambda \downarrow_{(i-2 k)}^{(i)}\right)+\sum_{a=i-2 k+1}^{i} \lambda_{a}^{\prime}} K_{\nu, \lambda \downarrow_{(i-2 k)}^{(i)}}(-1)
\end{aligned}
$$

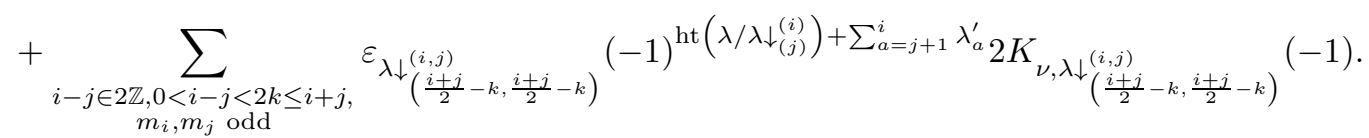

From now on, we suppose that $\lambda$ is a Jordan type of some nilpotent element in $\mathfrak{g}$ of type $B, C$, or $D$. Then case-by-case check shows that $\sum_{a=i-2 k+1}^{i} \lambda_{a}^{\prime} \equiv 0 \bmod 2$ for any choice of $i$ such that $m_{i}$ is odd. Similarly, we always have $\sum_{a=j+1}^{i} \lambda_{a}^{\prime} \equiv 0 \bmod 2$ for $i, j$ such that $i>j$ and $m_{i}, m_{j}$ are odd. On the other hand, if $m_{i}$ and $m_{j}$ are odd then we always have $i-j \in 2 \mathbb{Z}$. Therefore, we can simplify the above propositions a bit as follows. 
Theorem 10.5. Assume $\lambda=\left(1^{m_{1}} 2^{m_{2}} \cdots\right)$ is a partition of the Jordan type of some nilpotent element in $\mathfrak{g}$ of type $B, C$, or $D$ and let $k \geq 1$. Then we have

$$
\begin{aligned}
\mathcal{Q}_{\rho \cup(2 k)}^{\lambda}(-1)= & \sum_{i \geq k, m_{i} \geq 2} 2\left\lfloor\frac{m_{i}}{2}\right\rfloor \mathcal{Q}_{\rho}^{\lambda \downarrow_{(i-k, i-k)}^{(i, i)}}(-1) \\
+ & \sum_{i \geq 2 k, m_{i} \text { odd }}(-1)^{\mathrm{ht}\left(\lambda / \lambda \downarrow_{(i-2 k)}^{(i)}\right)} \mathcal{Q}_{\rho}^{\lambda \downarrow_{(i-2 k)}^{(i)}(-1)} \\
+ & \sum_{\substack{0<i-j<2 k \leq i+j, m_{i}, m_{j} \text { odd }}}(-1)^{\mathrm{ht}\left(\lambda / \lambda \downarrow_{(j)}^{(i)}\right)} 2 \mathcal{Q}_{\rho}^{\lambda \downarrow^{(i, j)}}
\end{aligned}
$$

Theorem 10.6. Under the same assumptions above, we have

$$
\begin{aligned}
& \varepsilon_{\lambda} \sum_{\mu \vdash n, \mu / \nu \text { border strip }}(-1)^{\mathrm{ht}(\mu / \nu)} K_{\mu, \lambda}(-1) \\
= & \sum_{i \geq k, m_{i} \geq 2} \varepsilon_{\lambda \downarrow_{(i-k, i-k)}^{(i, i)}} 2\left\lfloor\frac{m_{i}}{2}\right\rfloor K_{\nu, \lambda \downarrow_{(i-k, i-k)}^{(i, i)}}(-1) \\
& +\sum_{i \geq 2 k, m_{i} \text { odd }} \varepsilon_{\lambda \downarrow_{(i-2 k)}^{(i)}}(-1)^{\mathrm{ht}\left(\lambda / \lambda \downarrow_{(i-2 k)}^{(i)}\right)} K_{\nu, \lambda \downarrow_{(i-2 k)}^{(i)}}(-1) \\
& +\sum_{\substack{0<i-j<2 k \leq i+j, m_{i}, m_{j} \text { odd }}} \varepsilon_{\lambda \downarrow}^{\substack{(i, j) \\
\left(\frac{i+j}{2}-k, \frac{i+j}{2}-k\right)}}(-1)^{\mathrm{ht}\left(\lambda / \lambda \downarrow_{(j)}^{(i)}\right)} 2 K_{\nu, \lambda \downarrow}^{\left(\frac{i+j}{2}-k, \frac{i+j}{2}-k\right)}(-1) .
\end{aligned}
$$

\section{Proof of Main Theorem II}

We are ready to prove Main Theorem II] Indeed it is easily deduced from Theorem 7.12 , 8.5, and 10.5, as we see below.

Theorem 11.1. Suppse $G=S O_{2 n+1}(\mathbb{C})$ or $S p_{2 n}(\mathbb{C})$. Let $\mathfrak{S}_{n} \subset W$ be the maximal parabolic subgroup of $W$ and let $w_{\rho} \in \mathfrak{S}_{n}$ be an element of cycle type $\rho \vdash n$.

- If $G=S O_{2 n+1}(\mathbb{C})$, then $\operatorname{ch} \mathbf{T S p}(\lambda)\left(w_{\rho}\right)=\mathcal{Q}_{2 \rho \cup(1)}^{\lambda}(-1)$.

- If $G=S p_{2 n}(\mathbb{C})$, then $\operatorname{ch} \mathbf{T S p}(\lambda)\left(w_{\rho}\right)=\mathcal{Q}_{2 \rho}^{\lambda}(-1)$.

Proof. We proceed by induction on $l(\rho)$. If $\rho$ is a cycle, i.e. $\rho=(n)$, then Theorem 7.12 and Theorem 10.5 imply that

- if $G=S O_{2 n+1}(\mathbb{C})$, then

$$
\operatorname{ch} \mathbf{T S p}(\lambda)\left(w_{(n)}\right)=2 \delta_{\lambda,(n, n, 1)}+\delta_{\lambda,(2 n+1)}+\sum_{i>j>1 \text { odd }, i+j=2 n} 2 \delta_{\lambda,(i, j, 1)}=\mathcal{Q}_{(2 n, 1)}^{\lambda}(-1)
$$

- if $G=S p_{2 n}(\mathbb{C})$, then

$$
\operatorname{ch} \mathbf{T S p}(\lambda)\left(w_{(n)}\right)=2 \delta_{\lambda,(n, n)}+\delta_{\lambda,(2 n)}+\sum_{i>j>0} 2 \delta_{\lambda,(i, j)}=\mathcal{Q}_{(2 n)}^{\lambda}(-1)
$$


Thus the theorem holds. Otherwise, we set $\rho=(k) \cup \rho^{\prime}$ for some $\rho^{\prime} \vdash n-k$ and $1 \leq k<n$. If $G=S p_{2 n}(\mathbb{C})$ then we have

$$
\begin{aligned}
\operatorname{ch} \mathbf{T S p}(\lambda)\left(w_{\rho}\right)= & \sum_{i \geq k, m_{i} \geq 2} 2\left\lfloor\frac{m_{i}}{2}\right\rfloor \operatorname{ch} \mathbf{T S p}\left(\lambda \downarrow_{(i-k, i-k)}^{(i, i)}\right)\left(w_{\rho^{\prime}}\right) \\
& +\sum_{i \geq 2 k, m_{i} \text { odd }}(-1)^{\operatorname{ht}\left(\lambda /\left(\lambda \downarrow_{(i-2 k)}^{(i)}\right)\right)} \operatorname{ch~TSp}\left(\lambda \downarrow_{(i-2 k)}^{(i)}\right)\left(w_{\rho^{\prime}}\right) \\
& +\sum_{\substack{0<i-j<2 k \leq i+j \\
m_{i}, m_{j} \text { odd }}}(-1)^{\operatorname{ht}\left(\lambda /\left(\lambda \downarrow_{(j)}^{(i)}\right)\right)} 2 \operatorname{ch} \mathbf{T S p}\left(\lambda \downarrow_{\left.\frac{(i+j}{2}-k, \frac{i+j}{2}-k\right)}^{(i, j)}\right)\left(w_{\rho^{\prime}}\right) .
\end{aligned}
$$

By induction assumption, it is equal to

$$
\begin{aligned}
& \sum_{i \geq k, m_{i} \geq 2} 2\left\lfloor\frac{m_{i}}{2}\right\rfloor \mathcal{Q}_{2 \rho^{\prime}}^{\lambda \downarrow_{(i-k, i-k)}^{(i, i)}}(-1)+\sum_{i \geq 2 k, m_{i} \text { odd }}(-1)^{\mathrm{ht}\left(\lambda /\left(\lambda \downarrow_{(i-2 k)}^{(i)}\right)\right)} \mathcal{Q}_{2 \rho^{\prime}}^{\lambda \downarrow_{(-2 k)}^{(i)}}(-1) \\
& +\sum_{\substack{0<i-j<2 k \leq i+j, m_{i}, m_{j} \text { odd }}}(-1)^{\mathrm{ht}\left(\lambda /\left(\lambda \downarrow_{(j)}^{(i)}\right)\right)} 2 \mathcal{Q}_{2 \rho^{\prime}}^{\lambda \underbrace{(i, j)} \frac{i+j}{2}-k, \frac{i+j}{2}-k)}(-1) \\
& =\mathcal{Q}_{2 \rho^{\prime} \cup(2 k)}^{\lambda}(-1)=\mathcal{Q}_{2 \rho}^{\lambda}(-1) .
\end{aligned}
$$

If $G=S O_{2 n+1}(\mathbb{C})$, we simply replace $2 \rho^{\prime}, 2 \rho$ with $2 \rho^{\prime} \cup(1), 2 \rho \cup(1)$, respectively, and the equation is still valid. It suffices for the proof.

Theorem 11.2. Suppose $G=S O_{2 n}(\mathbb{C})$. Let $\mathfrak{S}_{n+}$ (resp. $\left.\mathfrak{S}_{n-}\right)$ be the maximal parabolic subgroup of $W$ which does not contain $s_{-}$(resp. $s_{+}$) and let $w_{\rho} \in \mathfrak{S}_{n \pm}$ be an element of cycle type $\rho$. If $\rho$ is even, then we write $w_{\rho \pm} \in \mathfrak{S}_{n \pm}$ to avoid ambiguity.

(1) If $\lambda$ is not very even and $\rho$ is not even, then $\operatorname{ch} \mathbf{T S p}(\lambda)\left(w_{\rho}\right)=\frac{1}{2} \mathcal{Q}_{2 \rho}^{\lambda}(-1)$.

(2) If $\lambda$ is very even and $\rho$ is not even, then $\operatorname{ch} \mathbf{T S p}(\lambda \pm)\left(w_{\rho}\right)=\frac{1}{2} \mathcal{Q}_{2 \rho}^{\lambda}(-1)$.

(3) If $\lambda$ is not very even and $\rho$ is even, then $\operatorname{ch} \mathbf{T S p}(\lambda)\left(w_{\rho \pm}\right)=\frac{1}{2} \mathcal{Q}_{2 \rho}^{\lambda}(-1)$.

(4) If $\lambda$ is very even and $\rho$ is even, then

$$
\operatorname{ch} \mathbf{T S p}(\lambda \pm)\left(w_{\rho \pm}\right)=\mathcal{Q}_{2 \rho}^{\lambda}(-1) \quad \text { and } \quad \operatorname{ch} \mathbf{T S p}(\lambda \mp)\left(w_{\rho \pm}\right)=0 .
$$

Proof. By the same argument as Theorem 11.1 using Theorem 8.5 and Theorem 10.5 we have

$$
\operatorname{ch} \widetilde{\mathbf{T S p}}(\lambda)\left(w_{\rho}\right)=\mathcal{Q}_{2 \rho}^{\lambda}(-1) .
$$

Now the statement follows from two observations: (1) if $\rho$ is not even or $\lambda$ is not very even, then $\operatorname{ch} \mathbf{T S p}(\lambda)\left(w_{\rho}\right)=\operatorname{ch} \mathbf{T S p}(\lambda)\left(\tau\left(w_{\rho}\right)\right)$, and (2) if $\rho$ is even and $\lambda$ is very even, then $\operatorname{ch} \mathbf{T S p}(\lambda \mp)\left(w_{\rho \pm}\right)=$ 0. (cf. Lemma 3.1)

By letting $\rho=\left(1^{n}\right)$ in Theorem 11.1 and 11.2, we obtain closed formulas for the Euler characteristic of Springer fibers.

Corollary 11.3. Let $N \in \mathfrak{g}$ be a nilpotent element of Jordan type $\lambda$.

(1) If $G=S O_{2 n+1}(\mathbb{C})$, then $\mathcal{X}\left(\mathcal{B}_{N}\right)=\mathcal{Q}_{\left(1^{1} 2^{n}\right)}^{\lambda}(-1)$. 
(2) If $G=S p_{2 n}(\mathbb{C})$, then $\mathcal{X}\left(\mathcal{B}_{N}\right)=\mathcal{Q}_{\left(2^{n}\right)}^{\lambda}(-1)$.

(3) If $G=S O_{2 n}(\mathbb{C})$, then $\mathcal{X}\left(\mathcal{B}_{N}\right)=\frac{1}{2} \mathcal{Q}_{\left(2^{n}\right)}^{\lambda}(-1)$.

\section{Proof of Main Theorem \for type $B$ and $C$}

Assume $G$ is of type $B_{n}$ or $C_{n}$ and define

$$
\Upsilon_{\lambda}:=\sum_{\mu \vdash|\lambda|} \varepsilon_{\lambda} \varepsilon_{\mu} K_{\mu, \lambda}(-1) \operatorname{ch} \chi^{\mu} .
$$

In this section we show that $\operatorname{ch} \mathbf{T S p}(\lambda)=\Upsilon_{\lambda}$, hence Main Theorem [1] Our strategy to prove it is as follows.

(1) $\Upsilon_{\lambda}$ satisfies the "restriction property" similar to Theorem 7.12 ,

(2) $\Upsilon_{\lambda}$ satisfies the "induction property" similar to [Lus04, see Proposition 12.4,

(3) Use "(upper) triangularity properties" of $\Upsilon_{\lambda}$ and total Springer representations.

12.1. Restriction property. First we show the following proposition that is an analogue to Theorem 7.12 .

Proposition 12.1. Assume $1 \leq k \leq n$. Let $\mathfrak{S}_{k} \times W^{\prime} \subset W$ be the maximal parabolic subgroup where $W^{\prime}$ is of the same type as $W$. If $c \in \mathfrak{S}_{k}$ is a $k$-cycle, then for $\lambda=\left(1^{m_{1}} 2^{m_{2}} \ldots\right)$ we have

$$
\begin{aligned}
\operatorname{Res}_{c \cdot W^{\prime}}^{W} \Upsilon_{\lambda}= & \sum_{i \geq k, m_{i} \geq 2} 2\left\lfloor\frac{m_{i}}{2}\right\rfloor \Upsilon_{\lambda \downarrow_{(i-k, i-k)}^{(i, i)}}+\sum_{i \geq 2 k, m_{i} \text { odd }}(-1)^{\mathrm{ht}\left(\lambda /\left(\lambda \downarrow_{(i-2 k)}^{(i)}\right)\right)} \Upsilon_{\lambda \downarrow_{(i-2 k)}^{(i)}} \\
& +\sum_{\substack{0<i-j<2 k \leq i+j, m_{i}, m_{j} \text { odd }}}(-1)^{\mathrm{ht}\left(\lambda /\left(\lambda \downarrow_{(j)}^{(i)}\right)\right)} 2 \Upsilon_{\lambda \downarrow_{\left(\frac{i+j}{2}-k, \frac{i+j}{2}-k\right)}^{(i, j)}} .
\end{aligned}
$$

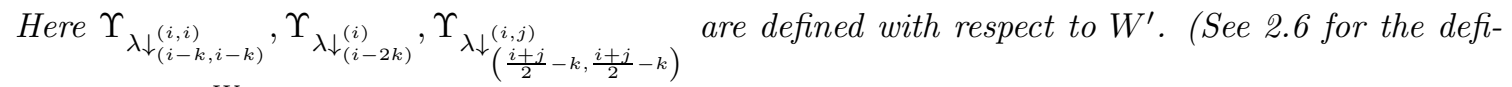
nition of $\operatorname{Res}_{c \cdot W^{\prime}}^{W}$.)

To that end, first note that the statement is equivalent to that

$$
\begin{aligned}
& \left\langle\operatorname{Res}_{c \cdot W^{\prime}}^{W} \Upsilon_{\lambda}, \operatorname{ch} \chi^{\nu}\right\rangle=\sum_{i \geq k, m_{i} \geq 2} 2\left\lfloor\frac{m_{i}}{2}\right\rfloor \varepsilon_{\nu} \varepsilon_{\lambda \downarrow_{(i-k, i-k)}^{(i, i)}} K_{\nu, \lambda \downarrow_{(i-k, i-k)}^{(i, i)}}(-1)
\end{aligned}
$$

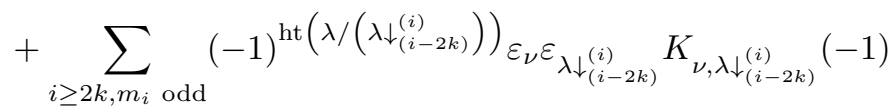

$$
\begin{aligned}
& +\sum_{\substack{0<i-j<2 k \leq i+j, m_{i}, m_{j} \text { odd }}}(-1)^{\mathrm{ht}\left(\lambda /\left(\lambda \downarrow_{(j)}^{(i)}\right)\right)} 2 \varepsilon_{\nu} \varepsilon_{\lambda \downarrow_{\left(\frac{i+j}{2}-k, \frac{i+j}{2}-k\right)}^{(i, j)}} K_{\nu, \lambda \downarrow_{\left(\frac{i+j}{2}-k, \frac{i+j}{2}-k\right)}^{(i, j)}}(-1)
\end{aligned}
$$

for all $\nu \vdash|\lambda|-2 k$ with minimal 2-core. On the other hand, we have

Lemma 12.2. For $f \in \mathcal{R}(W)$, we have

$$
\Psi\left(\operatorname{Res}_{c \cdot W^{\prime}}^{W} f\right)=p_{((k), \emptyset)}^{*,\langle,\rangle} \Psi(f)=\left(p_{k}(x)+p_{k}(y)\right)^{*,\langle,\rangle} \Psi(f)
$$


where $\Psi$ is defined in Section 9 .

Proof. It is an easy exercise.

Thus we have

$$
\begin{aligned}
\left\langle\operatorname{Res}_{c \cdot W^{\prime}}^{W} \Upsilon_{\lambda}, \operatorname{ch} \chi^{\nu}\right\rangle & =\left\langle\sum_{\mu \vdash|\lambda|} \varepsilon_{\lambda} \varepsilon_{\mu} K_{\mu, \lambda}(-1) \operatorname{Res}_{c^{\prime} \cdot W^{\prime}}^{W} \operatorname{ch} \chi^{\mu}, \operatorname{ch} \chi^{\nu}\right\rangle \\
& =\sum_{\mu \vdash|\lambda|} \varepsilon_{\lambda} \varepsilon_{\mu} K_{\mu, \lambda}(-1)\left\langle s_{\mu^{(0)}}(x) s_{\mu^{(1)}}(y), s_{\nu^{(0)}}(x) s_{\nu^{(1)}}(y)\left(p_{k}(x)+p_{k}(y)\right)\right\rangle .
\end{aligned}
$$

(As the formula is symmetric with respect to $x$ and $y$, this formula is valid for both type $B$ and C.) By the Murnaghan-Nakayama rule, we have

$$
\left\langle\operatorname{Res}_{c \cdot W^{\prime}}^{W} \Upsilon_{\lambda}, \operatorname{ch} \chi^{\nu}\right\rangle=\sum_{\mu} \varepsilon_{\lambda} \varepsilon_{\mu}(-1)^{\mathrm{ht}\left(\mu^{(0)} / \nu^{(0)}\right)+\mathrm{ht}\left(\mu^{(1)} / \nu^{(1)}\right)} K_{\mu, \lambda}(-1)
$$

where the sum is over all $\mu \vdash|\lambda|$ such that either $\mu^{(0)} / \nu^{(0)}$ is a border strip of size $k$ and $\mu^{(1)}=\nu^{(1)}$, or $\mu^{(1)} / \nu^{(1)}$ is a border strip of size $k$ and $\mu^{(0)}=\nu^{(0)}$. But this condition can be translated in a more direct way using the following lemma.

Lemma 12.3. Suppose that a partition $\nu \vdash|\lambda|-2 k$ with minimal 2-core is given.

(1) The mapping $\mu \mapsto\left(\mu^{(0)}, \mu^{(1)}\right)$ gives a bijection

$$
\{\mu \vdash|\lambda| \mid \mu / \nu \text { is a border strip of size } 2 k\} \rightarrow
$$

$\left\{\left(\alpha, \nu^{(1)}\right) \mid \alpha / \nu^{(0)}\right.$ is a border strip of size $\left.k\right\} \sqcup\left\{\left(\nu^{(0)}, \beta\right) \mid \beta / \nu^{(1)}\right.$ is a border strip of size $\left.k\right\}$.

(2) If $\mu / \nu$ is a border strip of size $2 k$, then

$$
b(\mu)+b(\nu)+\mathrm{ht}(\mu / \nu) \equiv \operatorname{ht}\left(\mu^{(0)} / \nu^{(0)}\right)+\mathrm{ht}\left(\mu^{(1)} / \nu^{(1)}\right) \bmod 2 .
$$

Proof. The first part is well-known and dates back to Sta50. In order to prove the second claim, we first fix $\mu$ and regard it as an infinite 01-sequences; first consider the Young diagram of $\mu$ and label horizontal line segments with 1 and vertical ones with 0 . Then we read the labels from southwest to northeast. For example, the partition $(6,4,2)$ is converted to

$$
\cdots 0,0,0,1,1,0,1,1,0,1,1,0,1,1,1, \cdots \text {. }
$$

We let $a_{1}=1$ to be the 1 that appears first in the sequence corresponding to $\mu$ and successively define $a_{2}, a_{3}, \cdots$ by reading the sequence from left to right. Also we let $a_{i}=0$ for $i \leq 0$. For example, if $\mu=(6,4,2)$ then we have

$$
1=a_{1}=a_{2}=a_{4}=a_{5}=a_{7}=a_{8}=a_{10}=a_{11}=\cdots, \quad \cdots=a_{-1}=a_{0}=a_{3}=a_{6}=a_{9}=0 .
$$

Then it is known that the 2 -quotient of $\mu$ corresponds to the following sequences

$$
\cdots, a_{-3}, a_{-1}, a_{1}, a_{3}, a_{5}, \cdots \quad \text { and } \quad \cdots, a_{-4}, a_{-2}, a_{0}, a_{2}, a_{4}, \cdots \text {. }
$$

If we remove a border strip of size $2 k$ to obtain $\nu$, then there exists $x \geq 1$ such that $a_{x}=1$, $a_{x+2 k}=0$ and $\nu$ corresponds to the 01-sequence

$$
\cdots, a_{-2}, a_{-1}, a_{0}, \cdots, a_{x-1}, 0, a_{x+1}, \cdots, a_{x+2 k-1}, 1, a_{x+2 k+1}, \cdots .
$$


In other words, we change $a_{x}, a_{x+2 k}$ to 0,1 , respectively, from the 01-sequence of $\mu$.

Let $j_{1}<\cdots<j_{r}$ be the collection of all indices of zeroes in $\left\{a_{x+1}, \cdots, a_{x+2 k-1}\right\}$. Then it is easy to see that ht $(\mu / \nu)=r$ and each row in the border strip $\mu / \nu$ has length $j_{1}-x, j_{2}-j_{1}, \cdots, j_{r}-$ $j_{r-1}, x+2 k-j_{r-1}$, respectively. Thus it is also clear that

$$
b(\mu)-b(\nu) \equiv\left(j_{1}-x\right)+\left(j_{3}-j_{2}\right)+\cdots=\left(j_{2}-j_{1}\right)+\left(j_{4}-j_{3}\right)+\cdots \quad \bmod 2
$$

since $|\mu / \nu|=2 k$ is even. It follows that

$$
\begin{aligned}
& \text { if } r \text { is even, } \quad b(\mu)+b(\nu) \equiv x+\sum_{a=1}^{r} j_{a}+(x+2 k) \equiv \sum_{a=1}^{r} j_{a} \bmod 2, \\
& \text { if } r \text { is odd, } \quad b(\mu)+b(\nu) \equiv x+\sum_{a=1}^{r} j_{a} \bmod 2 .
\end{aligned}
$$

This is equivalent to

$$
b(\mu)+b(\nu)+\operatorname{ht}(\mu / \nu) \equiv(x+1) r+\sum_{a=1}^{r} j_{a} \bmod 2 .
$$

On the other hand, by a similar reason $h t\left(\mu^{(0)} / \nu^{(0)}\right)+\mathrm{ht}\left(\mu^{(1)} / \nu^{(1)}\right)$ is the number of $j_{a}$ such that $j_{a} \equiv x \bmod 2$. Thus we may write

$$
\text { ht }\left(\mu^{(0)} / \nu^{(0)}\right)+\operatorname{ht}\left(\mu^{(1)} / \nu^{(1)}\right) \equiv \sum_{a=1}^{r}\left(x+j_{a}+1\right) \equiv(x+1) r+\sum_{a=1}^{r} j_{a} \bmod 2 .
$$

It suffices for the proof.

Using the lemma above, we may simplify (12.1) to the following form.

$$
\left\langle\operatorname{Res}_{c^{\prime} W^{\prime}}^{W} \Upsilon_{\lambda}, \operatorname{ch} \chi^{\nu}\right\rangle=\sum_{\mu} \varepsilon_{\lambda} \varepsilon_{\nu}(-1)^{\mathrm{ht}(\mu / \nu)} K_{\mu, \lambda}(-1)
$$

Here the sum is over all $\mu \vdash|\lambda|$ such that $\mu / \nu$ is a border strip of size $2 k$. Thus in order to prove Proposition 12.1 it suffices to show that

$$
\begin{aligned}
& \sum_{\mu \vdash|\lambda|, \mu / \nu \text { border strip }} \varepsilon_{\lambda}(-1)^{\mathrm{ht}(\mu / \nu)} K_{\mu, \lambda}(-1) \\
& =\sum_{i \geq k, m_{i} \geq 2} 2\left\lfloor\frac{m_{i}}{2}\right\rfloor \varepsilon_{\lambda \downarrow_{(i-k, i-k)}^{(i, i)}} K_{\nu, \lambda \downarrow_{(i-k, i-k)}^{(i, i)}}(-1) \\
& +\sum_{i \geq 2 k, m_{i} \text { odd }}(-1)^{\mathrm{ht}\left(\lambda /\left(\lambda \downarrow_{(i-2 k)}^{(i)}\right)\right)} \varepsilon_{\lambda \downarrow_{(i-2 k)}^{(i)}} K_{\nu, \lambda \downarrow_{(i-2 k)}^{(i)}}(-1)
\end{aligned}
$$

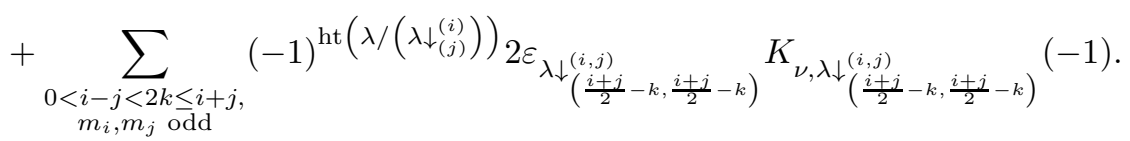

But this is exactly Theorem 10.6

12.2. Induction property. First we recall Lusztig's induction theorem for total Springer representations [Lus04, Theorem 1.3]. 
Proposition 12.4. Let $L \subset G$ be a Levi subgroup of some parabolic subgroup of $G$ and $W^{\prime}$ be the Weyl group of $L$, naturally identified with a subgroup of $W$. For $N \in \operatorname{Lie} L$, let $\mathcal{B}_{N}^{\prime}$ be the Springer fiber corresponding to $L$. Then we have an isomorphism

$$
H^{*}\left(\mathcal{B}_{N}\right) \simeq \operatorname{Ind}_{W^{\prime}}^{W} H^{*}\left(\mathcal{B}_{N}^{\prime}\right)
$$

of $W$-modules. Here we regard $H^{*}\left(\mathcal{B}_{N}^{\prime}\right)$ as the total Springer representation with respect to $W^{\prime}$.

We also claim that $\Upsilon_{\lambda}$ enjoys a similar property as follows.

Proposition 12.5. Assume $1 \leq k \leq n$. Let $\mathfrak{S}_{k} \times W^{\prime} \subset W$ be the maximal parabolic subgroup where $W^{\prime}$ is of the same type as $W$. Then we have

$$
\Upsilon_{\lambda \cup(k, k)}=\operatorname{Ind}_{\mathfrak{S}_{k} \times W^{\prime}}^{W} 1 \times \Upsilon_{\lambda}
$$

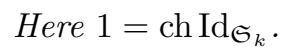

To that end, it is enough to show that for any $\nu \vdash|\lambda|+2 k$ with minimal 2-core we have

$$
\begin{gathered}
\varepsilon_{\lambda \cup(k, k)} \varepsilon_{\nu} K_{\nu, \lambda \cup(k, k)}(-1)=\left\langle\Upsilon_{\lambda \cup(k, k)}, \operatorname{ch} \chi^{\nu}\right\rangle \\
=\left\langle\operatorname{Ind}_{\mathfrak{S}_{k} \times W^{\prime}}^{W} 1 \times \Upsilon_{\lambda}, \operatorname{ch} \chi^{\nu}\right\rangle=\left\langle 1 \times \Upsilon_{\lambda}, \operatorname{Res}_{\mathfrak{S}_{k} \times W^{\prime}}^{W} \operatorname{ch} \chi^{\nu}\right\rangle .
\end{gathered}
$$

We claim the following.

Lemma 12.6. For $\mu \vdash|\lambda|,\left\langle\operatorname{Res}_{\mathfrak{S}_{k} \times W^{\prime}}^{W} \operatorname{ch} \chi^{\nu}, 1 \times \operatorname{ch} \chi^{\mu}\right\rangle=1$ if $\mu \subset \nu$ and $\nu / \mu$ can be covered by dominoes with no two dominoes intersecting the same column, and 0 otherwise.

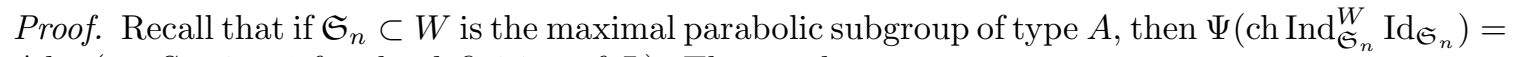
$\Delta h_{n}$ (see Section 9 for the definition of $\Psi$ ). Thus we have

$$
\begin{aligned}
& \left\langle\operatorname{Res}_{\mathfrak{S}_{k} \times W^{\prime}}^{W} \operatorname{ch} \chi^{\nu}, 1 \times \operatorname{ch} \chi^{\mu}\right\rangle=\left\langle\operatorname{ch} \chi^{\nu}, \operatorname{Ind}_{\mathfrak{S}_{k} \times W^{\prime}}^{W} 1 \times \operatorname{ch} \chi^{\mu}\right\rangle \\
& =\left\langle s_{\nu^{(0)}}(x) s_{\nu^{(1)}}(y), s_{\mu^{(0)}}(x) s_{\mu^{(1)}}(y) \Delta h_{k}\right\rangle=\left\langle s_{\nu^{(0)} / \mu^{(0)}}(x) s_{\nu^{(1)} / \mu^{(1)}}(y), \Delta h_{k}\right\rangle \\
& =\left\langle s_{\nu^{(0)} / \mu^{(0)}} s_{\nu^{(1)} / \mu^{(1)}}, h_{k}\right\rangle .
\end{aligned}
$$

using the adjunction of $\Delta$ and $\nabla$. (The formula is symmetric with respect to $x$ and $y$, thus this formula is valid for both type $B$ and $C$.) By vL00, Theorem 2.2.2] (see also [CL95]) this is the number of Yamanouchi domino tableaux of shape $\nu / \mu$ and weight $(k)$. One can easily check that this number is 1 if and only if the condition in the lemma is satisfied and otherwise 0, thus the result follows.

Thus we have

$$
\left\langle 1 \times \Upsilon_{\lambda}, \operatorname{Res}_{\mathfrak{S}_{k} \times W^{\prime}}^{W} \operatorname{ch} \chi^{\nu}\right\rangle=\sum_{\mu} \varepsilon_{\lambda} \varepsilon_{\mu} K_{\mu, \lambda}(-1)
$$

where the sum is over $\mu \vdash|\lambda|$ which satisfies the condition in Lemma 12.6. As $\varepsilon_{\lambda \cup(k, k)}=(-1)^{k} \varepsilon_{\lambda}$, in order to prove Proposition 12.5 it suffices to show that

$$
(-1)^{k} \varepsilon_{\nu} K_{\nu, \lambda \cup(k, k)}(-1)=\sum_{\mu} \varepsilon_{\mu} K_{\mu, \lambda}(-1)
$$

where the sum is over $\mu$ which satisfies the same condition above. Now we recall the following result of Lascoux, Leclerc, and Thibon. 
Lemma 12.7. Suppose $\lambda=\alpha \cup \beta \cup \beta$ for some partition $\alpha, \beta$. Then we have

$$
Q_{\lambda}^{\prime}(-1)=Q_{\alpha}^{\prime}(-1) \prod_{i \geq 1}\left(Q_{\left(i^{2}\right)}^{\prime}(-1)\right)^{\beta_{i}}
$$

Proof. This is a special case of [LLT94, Theorem 2.1] for $k=2$. The statement therein only considers the case when $\alpha$ is multiplicity-free (for $k=2$ ), but it is in fact equivalent to the formula above.

Therefore in particular, $Q_{\lambda \cup(k, k)}^{\prime}(-1)=Q_{\lambda}^{\prime}(-1) Q_{(k, k)}^{\prime}(-1)$. By [LLT94, Theorem 2.2], $Q_{(k, k)}^{\prime}(-1)=$ $(-1)^{k} s_{k}\left[p_{2}\right]$. Thus we have

$$
K_{\nu, \lambda \cup(k, k)}(-1)=\left\langle Q_{\lambda \cup(k, k)}^{\prime}(-1), s_{\nu}\right\rangle=(-1)^{k}\left\langle s_{k}\left[p_{2}\right] \sum_{\mu} K_{\mu, \lambda}(-1) s_{\mu}, s_{\nu}\right\rangle
$$

where the sum is over $\mu \vdash|\lambda|$ which satisfies the condition in Lemma 12.6] But [CL95, Theorem 4.1] says that $\epsilon_{2}(\nu / \mu)\left\langle s_{k}\left[p_{2}\right] s_{\mu}, s_{\nu}\right\rangle$ is the number of Yamanouchi domino tableaux of shape $\nu / \mu$ and weight $(k)$, i.e. 1 if and only if the condition in Lemma 12.6 is satisfied and otherwise 0. Therefore,

$$
K_{\nu, \lambda \cup(k, k)}(-1)=(-1)^{k} \sum_{\mu} \epsilon_{2}(\nu / \mu) K_{\mu, \lambda}(-1)
$$

where the sum is over $\mu \vdash|\lambda|$ which satisfies the condition in Lemma 12.6. Since $\epsilon_{2}(\nu / \mu)=\varepsilon_{\nu} \varepsilon_{\mu}$, this reads

$$
(-1)^{k} \varepsilon_{\nu} K_{\nu, \lambda \cup(k, k)}(-1)=\sum_{\mu} \varepsilon_{\mu} K_{\mu, \lambda}(-1)
$$

which proves (12.2). Thus Proposition 12.5 is proved.

12.3. Triangularity property. We proceed to the proof of Main Theorem $\square$ for type $B$ and $C$ by induction on $n=\operatorname{ss}-\operatorname{rank} G$. As we observed already, $\operatorname{TSp}(\lambda)$ and $\Upsilon_{\lambda}$ enjoy similar induction properties. In particular, if $\lambda=\mu \cup(k, k)$ for some $\mu$ and $k \geq 1$, then by induction assumption we have

$$
\operatorname{ch} \mathbf{T S p}(\lambda)=\operatorname{Ind}_{\mathfrak{S}_{k} \times W^{\prime}}^{W}(1 \times \operatorname{ch} \mathbf{T S p}(\mu))=\operatorname{Ind}_{\mathfrak{S}_{k} \times W^{\prime}}^{W}\left(1 \times \Upsilon_{\mu}\right)=\Upsilon_{\lambda}
$$

Thus it remains to consider the case when $\lambda$ corresponds to a distinguished nilpotent element. We fix such $\lambda$ from now on. 
Also, for any maximal proper parabolic subgroup $\mathfrak{S}_{k} \times W^{\prime} \subset W$ for $k \geq 1$ and $c \in \mathfrak{S}_{k}$ a $k$-cycle, by restriction properties and induction assumption we have

$$
\begin{aligned}
& \operatorname{Res}_{c \cdot W^{\prime}}^{W} \operatorname{ch} \mathbf{T S p}(\lambda)=\sum_{i \geq k, m_{i} \geq 2} 2\left\lfloor\frac{m_{i}}{2}\right\rfloor \operatorname{ch} \mathbf{T S p}\left(\lambda \downarrow_{(i-k, i-k)}^{(i, i)}\right) \\
& +\sum_{i \geq 2 k, m_{i} \text { odd }}(-1)^{\mathrm{ht}\left(\lambda /\left(\lambda \downarrow_{(i-2 k)}^{(i)}\right)\right)} \operatorname{ch} \mathbf{T S p}\left(\lambda \downarrow_{(i-2 k)}^{(i)}\right) \\
& +\sum_{\substack{0<i-j<2 k \leq i+j, m_{i}, m_{j} \text { odd }}}(-1)^{\mathrm{ht}\left(\lambda /\left(\lambda \downarrow_{(j)}^{(i)}\right)\right)} 2 \operatorname{ch} \mathbf{T S p}\left(\lambda \downarrow_{\left(\frac{i+j}{2}-k, \frac{i+j}{2}-k\right)}^{(i, j)}\right) . \\
& =\sum_{i \geq k, m_{i} \geq 2} 2\left\lfloor\frac{m_{i}}{2}\right\rfloor \Upsilon_{\lambda \downarrow_{(i-k, i-k)}^{(i, i)}} \\
& +\sum_{i \geq 2 k, m_{i} \text { odd }}(-1)^{\mathrm{ht}\left(\lambda /\left(\lambda \downarrow_{(i-2 k)}^{(i)}\right)\right)} \Upsilon_{\lambda \downarrow_{(i-2 k)}^{(i)}}
\end{aligned}
$$

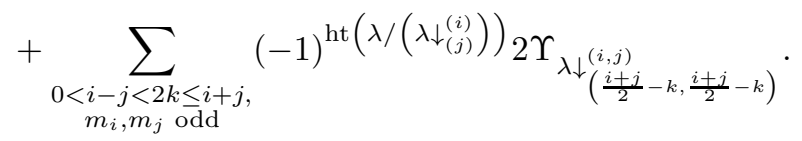

$$
\begin{aligned}
& =\operatorname{Res}_{c \cdot W^{\prime}}^{W} \Upsilon_{\lambda} .
\end{aligned}
$$

Thus ch $\mathbf{T S p}(\lambda)(w)=\Upsilon_{\lambda}(w)$ if $w \in W$ is contained in some maximal proper parabolic subgroup of $W$. In other words, $\operatorname{ch} \mathbf{T S p}(\lambda)-\Upsilon_{\lambda}$ is supported on the elements which are not contained in any proper parabolic subgroup. $w \in W$ is such an element if and only if the conjugacy class of $w$ is parametrized by $(\emptyset, \rho)$ for some partition $\rho \vdash n$. Thus for any $\mu \vdash n$ with minimal 2-core, we have

$$
\left\langle\operatorname{ch} \mathbf{T S p}(\lambda)-\Upsilon_{\lambda}, \operatorname{ch} \chi^{\mu}\right\rangle=\sum_{\rho \vdash n} \frac{1}{z_{\rho} 2^{l(\rho)}}\left(\operatorname{ch} \mathbf{T S p}(\lambda)\left(w_{(\emptyset, \rho)}\right)-\Upsilon_{\lambda}\left(w_{(\emptyset, \rho)}\right)\right) \operatorname{ch} \chi^{\mu}\left(w_{(\emptyset, \rho)}\right) .
$$

Here $z_{\rho} 2^{l(\rho)}$ is the size of the centralizer of $w_{(\emptyset, \rho)}$ in $W$. We observe the following (upper) triangularity properties of $\mathbf{T S p}(\lambda)$ and $\Upsilon_{\lambda}$.

Lemma 12.8. Let $\lambda, \mu$ be partitions with minimal 2-cores such that $|\lambda|=|\mu|$.

(1) If $\left\langle\mathbf{T S p}(\lambda), \chi^{\mu}\right\rangle \neq 0$, then $\lambda_{1} \leq \mu_{1}$.

(2) If $\left\langle\Upsilon_{\lambda}, \operatorname{ch} \chi^{\mu}\right\rangle \neq 0$, then $\lambda_{1} \leq \mu_{1}$.

Proof. The second part directly follows from the definition of $\Upsilon_{\lambda}$ and the fact that $K_{\mu, \lambda}(t) \neq 0 \Rightarrow$ $\lambda \leq \mu$. To show the first part, we let $\tilde{\mu} \vdash n$ be a partition such that $\chi^{\mu}$ corresponds to some local system on the nilpotent orbit of $\mathfrak{g}$ parametrized by $\tilde{\mu}$ under Springer correspondence. (Thus if $\chi^{\mu}$ corresponds to the trivial local system on some nilpotent orbit, then $\mu=\tilde{\mu}$.) By uppertriangularity property of Springer representations, we have $\lambda \leq \tilde{\mu}$, so that $\lambda_{1} \leq \tilde{\mu}_{1}$. Now it is an easy combinatorial exercise to see that $\tilde{\mu}_{1} \leq \mu_{1}$ by exploiting construction of Lusztig's symbols and its relation to Springer correspondence. (e.g. [Car93, Chapter 13.3]) 
Therefore, for $\mu \vdash n$ such that $\mu_{1}<\lambda_{1}$, we have $\left\langle\operatorname{ch} \mathbf{T S p}(\lambda)-\Upsilon_{\lambda}, \operatorname{ch} \chi^{\mu}\right\rangle=0$. Now we define an ad-hoc notation

$$
\operatorname{Res}^{W} \mathfrak{S}_{n} ": \mathcal{R}(W) \rightarrow \mathcal{R}\left(\mathfrak{S}_{n}\right) \quad \text { by } \quad\left(\operatorname{Res}^{W} \mathfrak{S}_{n} " f\right)\left(w_{\rho}\right)=f\left(w_{(\emptyset, \rho)}\right) .
$$

Then combined with (12.3), we only need to show the following statement.

$$
\left\{\operatorname{Res}^{W} \mathfrak{S}_{n} \text { " } \operatorname{ch} \chi^{\mu} \in \mathcal{R}\left(\mathfrak{S}_{n}\right) \mid \mu \vdash n, \mu_{1}<\lambda_{1}\right\} \text { spans } \mathcal{R}\left(\mathfrak{S}_{n}\right) .
$$

Recall that $\lambda$ is a Jordan type of some distinguished nilpotent element in $\mathfrak{g}$. For a partition $\mu$, we define $m_{\mu}:=\max \left(\left(\mu^{(0)}\right)_{1},\left(\mu^{(1)}\right)_{1}\right)$.

Lemma 12.9. Suppose $\mu$ is a partition with minimal 2-core.

(1) Suppose $|\mu|$ is odd. If $\mu_{1}$ is even, then $\mu_{1} \leq 2 m_{\mu}-2$. If $\mu_{1}$ is odd, then $\mu_{1} \leq 2 m_{\mu}+1$.

(2) Suppose $|\mu|$ is even. If $\mu_{1}$ is even, then $\mu_{1} \leq 2 m_{\mu}$. If $\mu_{1}$ is odd, then $\mu_{1} \leq 2 m_{\mu}-1$.

Proof. This is an easy combinatorial exercise using the definition of 2-cores.

We define $M \in \mathbb{N}$ as follows.

(1) If $G$ is of type $B_{n}$, set $M$ to be such that $M^{2}<2 n+1 \leq(M+1)^{2}$.

(2) If $G$ is of type $C_{n}$, set $M$ to be such that $(M-1) M<2 n \leq M(M+1)$.

Lemma 12.10. Let $\lambda$ be a Jordan type of a distinguished nilpotent element in $\mathfrak{g}$. Also suppose that a partition $\mu \vdash|\lambda|$ with minimal 2-core satisfies $m_{\mu}<M$. Then we have $\mu_{1}<\lambda_{1}$.

Proof. First assume that $G$ is of type $B$. Then since $\lambda$ is strict and each part is odd, we have $M^{2}<2 n+1=|\lambda| \leq \frac{\left(\lambda_{1}+1\right)^{2}}{4}$, thus $2 M<\lambda_{1}+1$. As $\lambda_{1}$ is odd, we have $2 M \leq \lambda_{1}-1$. Now by Lemma 12.9 we have

(1) if $\mu_{1}$ is even, then $\mu_{1} \leq 2 m_{\mu}-2<2 M-2 \leq \lambda_{1}-3<\lambda_{1}$, and

(2) if $\mu_{1}$ is odd, then $\mu_{1} \leq 2 m_{\mu}+1<2 M+1 \leq \lambda_{1}$.

Thus the result follows. Now assume that $G$ is of type $C$. Similarly, we have $(M-1) M<2 n=$ $|\lambda| \leq \frac{\lambda_{1}\left(\lambda_{1}+2\right)}{4}$, thus $2 M<\lambda_{1}+2$. As $\lambda_{1}$ is even, we have $2 M \leq \lambda_{1}$. Now by Lemma 12.9 we have

(1) if $\mu_{1}$ is even, $\mu_{1} \leq 2 m_{\mu}<2 M \leq \lambda_{1}$, and

(2) if $\mu_{1}$ is odd, $\mu_{1} \leq 2 m_{\mu}-1<2 M-1 \leq \lambda_{1}-1<\lambda_{1}$.

Thus the result follows.

We define

$$
\mathscr{L}:=\operatorname{sp}\left\{\operatorname{Res}^{W} \mathfrak{S}_{n} " \operatorname{ch} \chi^{\mu} \mid m_{\mu}<M\right\} \subset \mathcal{R}\left(\mathfrak{S}_{n}\right) .
$$

Then by Lemma 12.10, in order to prove (12.4) it suffices to show that $\mathscr{L}=\mathcal{R}\left(\mathfrak{S}_{n}\right)$. The following lemma provides a main tool to describe $\mathscr{L}$.

Lemma 12.11. If $G$ is of type $B$, then $\Psi\left(\operatorname{Res}^{W} \mathfrak{S}_{n^{\prime}}\right.$ " $\left.\operatorname{ch} \chi^{\mu}\right)=(-1)^{\left|\mu^{(1)}\right|} s_{\mu^{(0)}} s_{\left(\mu^{(1)}\right)^{\prime}}$. If $G$ is of type $C$, then $\Psi\left(\operatorname{Res}^{W} \mathbb{S}_{n}\right.$ " $\left.\operatorname{ch} \chi^{\mu}\right)=(-1)^{\left|\mu^{(0)}\right|} s_{\mu^{(1)}} s_{\left(\mu^{(0)}\right)^{\prime}}$ (See Section 9 for the definition of $\Psi$.) 
Proof. We only consider the case when $G$ is of type $B$ and the other case is totally analogous. By the definition of Res "W $\mathfrak{S}_{n}$ ", it is enough to show that for any $\rho \vdash n$ we have

$$
\operatorname{ch} \chi^{\mu}\left(w_{(\emptyset, \rho)}\right)=\left\langle s_{\mu^{(0)}}(x) s_{\mu^{(1)}}(y), p_{(\emptyset, \rho)}\right\rangle=\left\langle(-1)^{\left|\mu^{(1)}\right|} s_{\mu^{(0)}} s_{\left(\mu^{(1)}\right)^{\prime}}, p_{\rho}\right\rangle .
$$

Recall that $p_{(\emptyset, \rho)}=\prod_{i \geq 1}\left(p_{\rho_{i}}(x)-p_{\rho_{i}}(y)\right)$. If we apply the involution $\omega_{y}: \Lambda(x) \otimes \Lambda(y) \rightarrow \Lambda(x) \otimes$ $\Lambda(y): s_{\alpha}(x) s_{\beta}(y) \mapsto s_{\alpha}(x) s_{\beta^{\prime}}(y)$, then we have

$$
\begin{aligned}
& \left\langle s_{\mu^{(0)}}(x) s_{\mu^{(1)}}(y), p_{(\emptyset, \rho)}\right\rangle=\left\langle s_{\mu^{(0)}}(x) s_{\left(\mu^{(1)}\right)^{\prime}}(y), \prod_{i \geq 1}\left(p_{\rho_{i}}(x)+(-1)^{\rho_{i}} p_{\rho_{i}}(y)\right)\right\rangle \\
& =(-1)^{\left|\mu^{(1)}\right|}\left\langle s_{\mu^{(0)}}(x) s_{\left(\mu^{(1)}\right)^{\prime}}(y), \prod_{i \geq 1}\left(p_{\rho_{i}}(x)+p_{\rho_{i}}(y)\right)\right\rangle=(-1)^{\left|\mu^{(1)}\right|}\left\langle s_{\mu^{(0)}} s_{\left(\mu^{(1)}\right)^{\prime}}, p_{\rho}\right\rangle
\end{aligned}
$$

by adjunction of $\Delta$ and $\nabla$. Thus the result follows.

Therefore, we have

$$
\Psi(\mathscr{L})=\operatorname{sp}\left\{s_{\alpha} s_{\beta^{\prime}}|| \alpha|+| \beta \mid=n, \quad l(\alpha), l(\beta)<M\right\} \subset \Lambda^{n}
$$

and it remains to show that $\Psi(\mathscr{L})=\Lambda^{n}$.

Lemma 12.12. Let $M>0$ be such that $M^{2}>n$. Then $\Psi(\mathscr{L})=\Lambda^{n}$.

Proof. We introduce an increasing filtration

$$
\Lambda_{k}^{n}:=\mathbf{s p}\left\{s_{\lambda} \mid \sum_{i \geq M} \lambda_{i} \leq k\right\} \subset \Lambda^{n} .
$$

Clearly it exhausts $\Lambda^{n}$. We show $\Lambda_{k}^{n} \subset \Psi(\mathscr{L})$ by induction on $k \geq 0$. The case $k=0$ is straightforward, thus suppose $k \geq 1$. For any $\lambda$ such that $s_{\lambda} \in \Lambda_{k}^{n}-\Lambda_{k-1}^{n}$, we define $\alpha=\left(\lambda_{1}, \cdots, \lambda_{M-1}\right)$ and $\beta=\left(\lambda_{M}, \cdots, \lambda_{l(\lambda)}\right)^{\prime}$ so that $\lambda=\alpha \cup \beta^{\prime}$. Then $l(\beta)=\lambda_{M}<M$, since otherwise we have $|\lambda| \geq \sum_{i=1}^{M} \lambda_{i} \geq M \lambda_{M} \geq M^{2}>n$ which is absurd. We claim $s_{\alpha} s_{\beta^{\prime}}-s_{\lambda} \in \Lambda_{k-1}^{n}$. Indeed, if $\left\langle s_{\alpha} s_{\beta^{\prime}}, s_{\mu}\right\rangle \neq 0$ for some $\mu \vdash n$, then clearly $\alpha \subset \mu$. Thus we have $\sum_{i \geq M} \mu_{i} \leq k$ and the equality holds if and only if $\mu_{i}=\alpha_{i}$ for $1 \leq i<M$. Now the combinatorial description of the LittlewoodRichardson rule (e.g. Sta86, Theorem A1.3.3]) implies that

$$
s_{\mu} \in \Lambda_{k}^{n}-\Lambda_{k-1}^{n}, \quad\left\langle s_{\alpha} s_{\beta^{\prime}}, s_{\mu}\right\rangle \neq 0 \Rightarrow \mu=\lambda
$$

and also $\left\langle s_{\alpha} s_{\beta^{\prime}}, s_{\lambda}\right\rangle=1$. Thus the result follows.

Lemma 12.13. Let $n \geq 2$. Then $M^{2}>n$ except when $G$ is of type $B_{4}$.

Proof. Easy.

This finishes the proof except when $n=1$ or $G=S O_{9}(\mathbb{C})$. If $n=1$, then the statement can be easily checked by direct calculation. If $G=S O_{9}(\mathbb{C})$, the argument above does not work only when $\lambda=(5,3,1)$. However, direct calculation shows that we have

$$
\operatorname{ch} \mathbf{T S p}((5,3,1))=\operatorname{ch}\left(\chi^{(5,3,1)} \oplus \chi^{(5,4)} \oplus \chi^{(6,2,1)} \oplus \chi^{(7,1,1)} \oplus \chi^{(9)}\right)=\Upsilon_{(5,3,1)},
$$

and it completes the proof. 


\section{Proof of Main Theorem If for type $D$}

It remains to handle the case when $G$ is of type $D_{n}$ for $n \geq 2$. Here, we define

$$
\widetilde{\Upsilon}_{\lambda}:=\sum_{\substack{\mu \vdash|\lambda| \\ \text { not very even }}} \varepsilon_{\lambda} \varepsilon_{\mu} K_{\mu, \lambda}(-1) \operatorname{ch} \chi^{\mu}+\sum_{\substack{\mu \vdash|\lambda| \\ \text { very even }}} \varepsilon_{\lambda} K_{\mu, \lambda}(-1) \operatorname{ch}\left(\chi^{\mu+} \oplus \chi^{\mu-}\right) .
$$

(Note that $\varepsilon_{\mu}=1$ if $\mu$ is very even.)

13.1. $\operatorname{ch} \widetilde{\mathbf{T S p}}(\lambda)=\widetilde{\Upsilon}_{\lambda}$. The Weyl group $W$ of $G$ can be regarded naturally as a subgroup of $W\left(C_{n}\right)$. If we write $\Upsilon_{C, \lambda}:=\sum_{\mu \vdash|\lambda|} \varepsilon_{\lambda} \varepsilon_{\mu} K_{\mu, \lambda}(-1) \operatorname{ch} \chi_{C}^{\mu}$ where $\chi_{C}^{\mu}$ is the irreducible character of $W\left(C_{n}\right)$ parametrized by $\mu \vdash 2 n$, then it is clear that $\widetilde{\Upsilon}_{\lambda}=\operatorname{Res}_{W}^{W\left(C_{n}\right)} \Upsilon_{C, \lambda}$.

We first prove ch $\widetilde{\mathbf{T S p}}(\lambda)=\widetilde{\Upsilon}_{\lambda}$. Our strategy is similar to the previous section; we first state and prove the restriction and induction properties of $\widetilde{\Upsilon}_{\lambda}$.

Proposition 13.1. Assume $1 \leq k \leq n$. Let $\mathfrak{S}_{k} \times W^{\prime} \subset W$ be a parabolic subgroup where $W^{\prime}$ is of the same type as $W$. If $1 \leq k \leq n-2$, then we choose $W^{\prime}$ such that $\mathfrak{S}_{k} \times W^{\prime}$ becomes a maximal parabolic subgroup of $W$. ( $W^{\prime}$ is trivial if $k=n-1$ or $n$.) If $c \in \mathfrak{S}_{k}$ is a $k$-cycle and $\lambda=\left(1^{m_{1}} 2^{m_{2}} \ldots\right)$ then we have

$$
\begin{aligned}
\operatorname{Res}_{c \cdot W^{\prime}}^{W} \widetilde{\Upsilon}_{\lambda}= & \sum_{i \geq k, m_{i} \geq 2} 2\left\lfloor\frac{m_{i}}{2}\right\rfloor \widetilde{\Upsilon}_{\lambda \downarrow_{(i-k, i-k)}^{(i, i)}}(-1)^{\mathrm{ht}\left(\lambda /\left(\lambda \downarrow_{(i-2 k)}^{(i)}\right)\right)} \widetilde{\Upsilon}_{\lambda \downarrow_{(i-2 k)}^{(i)}} \\
& +\sum_{i \geq 2 k, m_{i} \text { odd }}(-1)^{\mathrm{ht}\left(\lambda /\left(\lambda \downarrow_{(j)}^{(i)}\right)\right)} 2 \widetilde{\Upsilon}_{\lambda \downarrow_{\left(\frac{i+j}{2}-k, \frac{i+j}{2}-k\right)}^{(i, j)}} . \\
& +\sum_{\substack{0<i-j<2 k \leq i+j \\
m_{i}, m_{j} \text { odd }}} .
\end{aligned}
$$

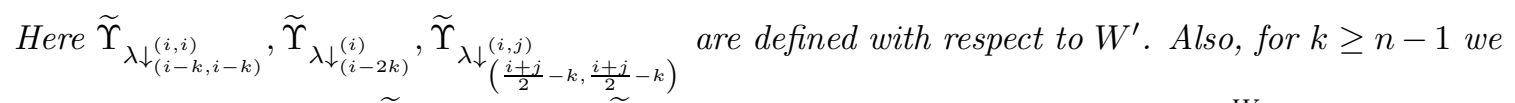
use the convention that $\widetilde{\Upsilon}_{(1,1)}=2$ and $\widetilde{\Upsilon}_{\emptyset}=1$. (See 2.6 for the definition of $\operatorname{Res}_{c^{\prime} \cdot W^{\prime}}$.)

Proof. Let $W^{\prime \prime} \subset W\left(C_{n}\right)$ be the parabolic subgroup of type $C_{n-k}$ such that $W^{\prime \prime} \cap W=W^{\prime}$. Then Proposition 12.1 shows that

$$
\begin{aligned}
\operatorname{Res}_{C \cdot W^{\prime \prime}}^{W\left(C_{n}\right)} \Upsilon_{C, \lambda}= & \sum_{i \geq k, m_{i} \geq 2} 2\left\lfloor\frac{m_{i}}{2}\right\rfloor \Upsilon_{C, \lambda \downarrow_{(i-k, i-k)}^{(i, i)}}(-1)^{\mathrm{ht}\left(\lambda /\left(\lambda \downarrow_{(i-2 k)}^{(i)}\right)\right)} \Upsilon_{C, \lambda \downarrow_{(i-2 k)}^{(i)}} \\
& +\sum_{i \geq 2 k, m_{i} \text { odd }}(-1)^{\mathrm{ht}\left(\lambda /\left(\lambda \downarrow_{(j)}^{(i)}\right)\right)} 2 \Upsilon_{C, \lambda \downarrow} \sum_{\substack{\left(\frac{i+j}{2}-k, \frac{i+j}{2}-k\right) \\
0<i-j<2 k \leq i+j \\
m_{i}, m_{j} \text { odd }}} .
\end{aligned}
$$

Now the result follows by applying $\operatorname{Res}_{W^{\prime}}^{W^{\prime \prime}}$ on both sides. (Note that $\operatorname{Res}_{\{*\}}^{W\left(C_{1}\right)} \Upsilon_{C,(1,1)}=2$ and $\Upsilon_{C, \emptyset}=1$.) 
Proposition 13.2. Assume $1 \leq k \leq n-2$. Let $\mathfrak{S}_{k} \times W^{\prime} \subset W$ be a maximal parabolic subgroup where $W^{\prime}$ is of the same type as $W$. Then we have

$$
\widetilde{\Upsilon}_{\lambda \cup(k, k)}=\operatorname{Ind}_{\mathfrak{S}_{k} \times W^{\prime}}^{W} 1 \times \widetilde{\Upsilon}_{\lambda} .
$$

Here $1=\operatorname{ch~Id~}_{\mathfrak{S}_{k}}$. Furthermore, we have

$$
\widetilde{\Upsilon}_{(n-1, n-1,1,1)}=\operatorname{Ind}_{\mathfrak{S}_{n-1}}^{W} 2, \quad \widetilde{\Upsilon}_{(n, n)}=\operatorname{Ind}_{\mathfrak{S}_{n+}}^{W} 1+\operatorname{Ind}_{\mathfrak{S}_{n-}}^{W} 1 .
$$

Proof. We define $W^{\prime \prime} \subset W\left(C_{n}\right)$ as before. We know that $\Upsilon_{C, \lambda \cup(k, k)}=\operatorname{Ind}_{\mathfrak{S}_{k} \times W^{\prime \prime}}^{W\left(C_{n}\right)} 1 \times \Upsilon_{C, \lambda}$. If we apply $\operatorname{Res}_{W}^{W\left(C_{n}\right)}$ on both sides then we have

$$
\begin{aligned}
\widetilde{\Upsilon}_{\lambda \cup(k, k)} & =\operatorname{Res}_{W}^{W\left(C_{n}\right)} \Upsilon_{C, \lambda \cup(k, k)}=\operatorname{Res}_{W}^{W\left(C_{n}\right)} \operatorname{Ind}_{\mathfrak{S}_{k} \times W^{\prime \prime}}^{W\left(C_{n}\right)} 1 \times \Upsilon_{C, \lambda} \\
& =\operatorname{Ind}_{\mathfrak{S}_{k} \times W^{\prime}}^{W} \operatorname{Res}_{\mathfrak{S}_{k} \times W^{\prime}}^{\mathfrak{S}_{k} \times W^{\prime \prime}} 1 \times \Upsilon_{C, \lambda}=\operatorname{Ind}_{\mathfrak{S}_{k} \times W^{\prime}}^{W} 1 \times \widetilde{\Upsilon}_{\lambda}
\end{aligned}
$$

by Mackey's formula. Similarly, we have

$$
\begin{aligned}
\widetilde{\Upsilon}_{(n-1, n-1,1,1)} & =\operatorname{Res}_{W}^{W\left(C_{n}\right)} \Upsilon_{C,(n-1, n-1,1,1)}=\operatorname{Res}_{W}^{W\left(C_{n}\right)} \operatorname{Ind}_{\mathfrak{S}_{n-1} \times W\left(C_{1}\right)}^{W\left(C_{n}\right)} 1 \times \Upsilon_{C,(1,1)} \\
& =\operatorname{Ind}_{\mathfrak{S}_{n-1}}^{W} \operatorname{Res}_{\mathfrak{S}_{n-1} \times W\left(C_{1}\right)}^{\mathfrak{S}_{n-1}} 1 \times \Upsilon_{C,(1,1)}=\operatorname{Ind}_{\mathfrak{S}_{n-1}}^{W} 2, \\
\widetilde{\Upsilon}_{(n, n)} & =\operatorname{Res}_{W}^{W\left(C_{n}\right)} \Upsilon_{C,(n, n)}=\operatorname{Res}_{W}^{W\left(C_{n}\right)} \operatorname{Ind}_{\mathfrak{S}_{n}}^{W\left(C_{n}\right)} 1=\operatorname{Ind}_{\mathfrak{S}_{n+}}^{W} 1+\operatorname{Ind}_{\mathfrak{S}_{n-}}^{W} 1 .
\end{aligned}
$$

Similarly to the previous section, by Proposition 13.2 , in order to prove ch $\widetilde{\mathbf{T S p}}(\lambda)=\widetilde{\Upsilon}_{\lambda}$ it suffices to consider the case when $\lambda$ corresponds to a distinguished nilpotent element. We fix such $\lambda$ from now on. Also by Proposition 13.1, we only need to show that ch $\widetilde{\mathbf{T S p}}(\lambda)(w)=\widetilde{\Upsilon}_{\lambda}(w)$ for $w \in W$ parametrized by $(\emptyset, \rho)$ for some $\rho \vdash n$. To that end we follow the arguments in the previous section.

Lemma 13.3. (1) $\lambda_{1} \leq \mu_{1}$ if the following holds: $\mu$ is not very even and $\left\langle\widetilde{\mathbf{T S p}}(\lambda), \chi^{\mu}\right\rangle \neq 0$, or $\mu$ is very even and either $\left\langle\widetilde{\mathbf{T S p}}(\lambda), \chi^{\mu+}\right\rangle \neq 0$ or $\left\langle\widetilde{\mathbf{T S p}}(\lambda), \chi^{\mu-}\right\rangle \neq 0$.

(2) $\lambda_{1} \leq \mu_{1}$ if the following holds: $\mu$ is not very even and $\left\langle\widetilde{\Upsilon}_{\lambda}, \operatorname{ch} \chi^{\mu}\right\rangle \neq 0$, or $\mu$ is very even and either $\left\langle\widetilde{\Upsilon}_{\lambda}, \operatorname{ch} \chi^{\mu+}\right\rangle \neq 0$ or $\left\langle\widetilde{\Upsilon}_{\lambda}, \operatorname{ch} \chi^{\mu-}\right\rangle \neq 0$.

Proof. It can be proved similarly to Lemma 12.8 ,

Remark. One needs to be careful here since $\chi^{\mu}=\chi^{\nu}$ does not necessarily mean $\mu=\nu$. In fact, if $\mu, \nu \vdash 2 n$ are partitions such that $\mu^{(c)}=\nu^{(c)}=\emptyset, \mu^{(0)}=\nu^{(1)}$, and $\mu^{(1)}=\nu^{(0)}$, then $\chi^{\mu}=\chi^{\nu}$ but $\mu \neq \nu$ unless $\mu$ is very even. However, the lemma above still remains valid; one may find the following fact useful.

Lemma 13.4. Suppose $\mu, \nu \vdash 2 n$ are partitions such that $\mu^{(c)}=\nu^{(c)}=\emptyset, \mu^{(0)}=\nu^{(1)}$, and $\mu^{(1)}=\nu^{(0)}$. Then there exists $r \in \mathbb{N}$ such that $\left\{\mu_{1}, \nu_{1}\right\}$ equals either $\{2 r\}$ or $\{2 r-1,2 r\}$. In particular, $\max \left\{\mu_{1}, \nu_{1}\right\}$ is always even. 
Recall that we defined $m_{\mu}:=\max \left(\left(\mu^{(0)}\right)_{1},\left(\mu^{(1)}\right)_{1}\right)$. We set $M \in \mathbb{N}$ such that $(M-1)^{2}<2 n \leq$ $M^{2}$. We also let $M_{C} \in \mathbb{N}$ such that $\left(M_{C}-1\right) M_{C}<2 n \leq M_{C}\left(M_{C}+1\right)$ as in the previous section for type $C$.

Lemma 13.5. Let $\lambda$ be a Jordan type of a distinguished nilpotent element in $\mathfrak{g}$. Also suppose we are given a partition $\mu \vdash 2 n$ such that $m_{\mu}<M$. Then we have $\mu_{1}<\lambda_{1}$.

Proof. Since $\lambda$ is strict and each part is odd, we have $(M-1)^{2}<2 n=|\lambda| \leq \frac{\left(\lambda_{1}+1\right)^{2}}{4}$, thus $2 M-2<\lambda_{1}+1$. As $\lambda_{1}$ is odd, we have $2 M \leq \lambda_{1}+1$. Now by Lemma 12.9 we have

(1) if $\mu_{1}$ is even, then $\mu_{1} \leq 2 m_{\mu}<2 M \leq \lambda_{1}+1$, and

(2) if $\mu_{1}$ is odd, then $\mu_{1} \leq 2 m_{\mu}-1<2 M-1 \leq \lambda_{1}$.

In the first case, as $\mu_{1}$ is even we also have $\mu_{1}<\lambda_{1}$. Thus the result follows.

Lemma 13.6. For $n \geq 2, M^{2} \geq M_{C}^{2}>n$.

Proof. Easy.

Now we prove ch $\widetilde{\mathbf{T S p}}(\lambda)=\widetilde{\Upsilon}_{\lambda}$. First set $\mathscr{P} \subset W\left(C_{n}\right)$ to be the union of conjugacy classes in $W\left(C_{n}\right)$ parametrized by $(\emptyset, \rho)$ for some $\rho \vdash n$. Also we let $\mathscr{P}_{e}:=\mathscr{P} \cap W$. Then $\mathscr{P}_{e}$ is the union of conjugacy classes in $W$ parametrized by $(\emptyset, \rho)$ for some $\rho \vdash n$ such that $l(\rho)$ is even. We denote by $\mathcal{R}(\mathscr{P}) \subset \mathcal{R}\left(W\left(C_{n}\right)\right)$ (resp. $\mathcal{R}\left(\mathscr{P}_{e}\right) \subset \mathcal{R}(W)$ ) the set of class functions of $W\left(C_{n}\right)$ (resp. $W$ ) supported on $\mathscr{P}\left(\right.$ resp. $\left.\mathscr{P}_{e}\right)$.

If we follow the argument in the previous section, we need to show that

$$
\left\{\operatorname{ch} \chi^{\mu}{\mid \mathscr{P}_{e}}_{e} \mid \mu \text { not very even, } \mu_{1}<\lambda_{1}\right\} \cup\left\{\left.\operatorname{ch} \chi^{\mu \pm}\right|_{\mathscr{P}_{e}} \mid \mu \text { very even, } \mu_{1}<\lambda_{1}\right\}
$$

spans $\mathcal{R}\left(\mathscr{P}_{e}\right)$. But we already know that $\left\{\left.\operatorname{ch} \chi_{C}^{\mu}\right|_{\mathscr{P}} \mid m_{\mu}<M_{C}\right\}$ spans $\mathcal{R}(\mathscr{P})$ by Lemma 12.12, By taking the restriction to $\mathscr{P}_{e}$, we see that

$\left\{\left.\operatorname{ch} \chi^{\mu}\right|_{\mathscr{P}_{e}} \mid \mu\right.$ not very even, $\left.m_{\mu}<M_{C}\right\} \cup\left\{\left.\operatorname{ch}\left(\chi^{\mu+} \oplus \chi^{\mu-}\right)\right|_{\mathscr{P}_{e}} \mid \mu\right.$ very even, $\left.m_{\mu}<M_{C}\right\}$

spans $\mathcal{R}\left(\mathscr{P}_{e}\right)$. Now the result easily follows from Lemma 13.5 and 13.6.

13.2. Very even case. If $\lambda$ is not very even, then the identity ch $\widetilde{\mathbf{T S p}}(\lambda)=\widetilde{\Upsilon}_{\lambda}$ directly implies Main Theorem 【 as $\widetilde{\mathbf{T S p}}(\lambda)=\mathbf{T S p}(\lambda) \oplus \mathbf{T S p}(\lambda)$. Even when $\lambda$ is very even, it proves Main Theorem 【 modulo the multiplicities of $\chi^{\mu \pm}$ for $\mu$ very even. Here, we fill this gap and finish the proof.

Suppose $n$ is even and $\lambda, \mu \vdash n / 2$ are given. We define

$$
\Theta_{\lambda}:=\operatorname{ch} \mathbf{T S p}((2 \lambda \cup 2 \lambda)+)-\operatorname{ch} \mathbf{T S p}((2 \lambda \cup 2 \lambda)-) .
$$

Then we claim the following. Note that it suffices to complete the proof of Main Theorem $\square$.

Proposition 13.7. $\left\langle\Theta_{\lambda}, \operatorname{ch} \chi^{(2 \mu \cup 2 \mu)+}\right\rangle=-\left\langle\Theta_{\lambda}, \operatorname{ch} \chi^{(2 \mu \cup 2 \mu)-}\right\rangle=K_{\mu, \lambda}$, where $K_{\mu, \lambda}=K_{\mu, \lambda}(1)$ is the Kostka number. 
Proof. Set $m_{\mu, \lambda}:=\left\langle\Theta_{\lambda}, \operatorname{ch} \chi^{(2 \mu \cup 2 \mu)+}\right\rangle=-\left\langle\Theta_{\lambda}, \operatorname{ch} \chi^{(2 \mu \cup 2 \mu)-}\right\rangle$. Since $\left\langle\Theta_{\lambda}, \operatorname{ch} \chi^{\alpha}\right\rangle=0$ if $\alpha$ is not very even, we have

$$
\left\langle\Theta_{\lambda}, \Theta_{\mu}\right\rangle=2 \sum_{\nu \vdash n / 2} m_{\nu, \lambda} m_{\nu, \mu} .
$$

Assuming the proposition, it should be the same as $2 \sum_{\nu \vdash n / 2} K_{\nu, \lambda} K_{\nu, \mu}=2\left\langle h_{\lambda}, h_{\mu}\right\rangle$. As the matrix $\left\{m_{\mu, \lambda}\right\}$ is upper-unitriangular (which comes from the upper-triangularity property of Springer representations), the proposition is in fact equivalent to the identity $\left\langle\Theta_{\lambda}, \Theta_{\mu}\right\rangle=2\left\langle h_{\lambda}, h_{\mu}\right\rangle$ for any $\lambda, \mu \vdash n / 2$.

By Theorem 11.2 we have

$$
\left\langle\Theta_{\lambda}, \Theta_{\mu}\right\rangle=2 \sum_{\rho \vdash n / 2} \frac{1}{z_{\rho} 2^{2 l(\rho)}} \mathcal{Q}_{4 \rho}^{2 \lambda \cup 2 \lambda}(-1) \mathcal{Q}_{4 \rho}^{2 \mu \cup 2 \mu}(-1)=2 \sum_{\rho \vdash n / 2} \frac{1}{z_{\rho}}\left\langle p_{2 \rho}, h_{2 \lambda}\right\rangle\left\langle p_{2 \rho}, h_{2 \mu}\right\rangle
$$

where the last equality is deduced from [LLT94, Theorem 3.4]. By adjunction of the Frobenius and Verschiebung operators it is equal to

$$
\begin{aligned}
2 \sum_{\rho \vdash n / 2} \frac{1}{z_{\rho}}\left\langle p_{\rho}\left[p_{2}\right], h_{2 \lambda}\right\rangle\left\langle p_{\rho}\left[p_{2}\right], h_{2 \mu}\right\rangle & =2 \sum_{\rho \vdash n / 2} \frac{1}{z_{\rho}}\left\langle p_{\rho}, \mathbb{V}_{2}\left(h_{2 \lambda}\right)\right\rangle\left\langle p_{\rho}, \mathbb{V}_{2}\left(h_{2 \mu}\right)\right\rangle \\
& =2 \sum_{\rho \vdash n / 2} \frac{1}{z_{\rho}}\left\langle p_{\rho}, h_{\lambda}\right\rangle\left\langle p_{\rho}, h_{\mu}\right\rangle,
\end{aligned}
$$

which is clearly the same as $2\left\langle h_{\lambda}, h_{\mu}\right\rangle$. Thus the result follows.

\section{REFERENCES}

[Car93] Carter, R. W., Finite Groups of Lie Type: Conjugacy Classes and Complex Characters, Wiley Classics Library, no. 48, Wiley, 1993.

[CL95] Carré, C. and Leclerc, B., Splitting the square of a Schur function into its symmetric and antisymmetric parts, J. Algebraic Combin. 4 (1995), 201-231.

[dCLP88] de Concini, C., Lusztig, G., and Procesi, C., Homology of the zero-set of a nilpotent vector field on a flag manifold, Journal of the AMS 1 (1988), no. 1, 15-34.

[DL76] Deligne, P. and Lusztig, G., Representations of reductive groups over a finite field, Ann. of Math. 103 (1976), 103-161.

[Gre55] Green, J. A., The characters of the finite general linear groups, Trans. Amer. Math. Soc. (1955), no. 2, $402-447$.

[HS79] Hotta, R. and Shimomura, N., The fixed point subvarieties of unipotent transformations on generalized flag varieties and the Green functions: Combinatorial and cohomological treatments centering $G L_{n}$, Math. Ann. 241 (1979), 193-208.

[Kaz77] Kazhdan, D., Proof of Springer's hypothesis., Israel J. Math. 28 (1977), no. 4, 272-286.

[Kim17] Kim, D., Euler characteristic of Springer fibers, To appear in Transform. Groups (2017).

[LLT94] Lascoux, A., Leclerc, B., and Thibon, J.-Y., Green polynomials and Hall-Littlewood functions at roots of unity, European J. Combin. 15 (1994), 173-180.

[Lus79] Lusztig, G., A class of irreducible representations of a Weyl group, Indagationes Mathematicae (Proceedings), vol. 82, 1979, pp. 323-335.

[Lus81] _ Green polynomials and singularities of unipotent classes, Adv. Math. 42 (1981), 169-178.

[Lus85] — Character sheaves II, Adv. Math. 57 (1985), 226-265.

[Lus86] Character sheaves, V, Adv. Math. 61 (1986), 103-155.

[Lus04] $\quad$ An induction theorem for Springer's representations, Representation Theory of Algebraic Groups and Quantum Groups, Adv. Stud. Pure Math., no. 40, 2004, pp. 253-259.

[Mac95] Macdonald, I. G., Symmetric Functions and Hall Polynomials, 2nd ed., Oxford mathematical monographs, Oxford University Press, 1995. 
[Sho79] Shoji, T., On the Springer representations of the Weyl groups of classical algebraic groups, Comm. Algebra 7 (1979), no. 16, 1713-1745.

[Sho83] _ On the Green polynomials of classical groups, Invent. Math 74 (1983), 239-267.

[Sho88] Geometry of orbits and Springer correspondence, Astérisque 168 (1988), 61-140.

[Spr71] Springer, T. A., Generalization of Green's polynomials, Representation Theory of Finite Groups and Related Topics, Proceedings of symposia in pure mathematics, vol. XXI, American Mathematical Society, 1971, pp. 149-153.

[Spr76] _ Trigonometric sums, Green functions of finite groups and representations of Weyl groups, Invent. Math. 36 (1976), 173-207.

[SS70] Springer, T. A. and Steinberg, R., Conjugacy classes, Seminar on algebraic groups and related finite groups: Held at the Institute for Advanced Study, Princeton/NJ, 1968/69, Lecture Notes in Mathematics, vol. 131, Springer-Verlag, 1970, pp. 167-266.

[Sta50] Staal, R. A., Star diagrams and the symmetric group, Canad. J. Math. 2 (1950), 79-92.

[Sta86] Stanley, R. P., Enumerative Combinatorics, vol. 2, Cambridge Studies in Advanced Mathematics, no. 62, Cambridge University Press, 1986.

[vL89] van Leeuwen, M. A. A., A Robinson-Schensted algorithm in the geometry of flags for classical groups, Ph.D. thesis, Rijksuniversiteit te Utrecht, 1989.

[vL00] S Some bijective correspondences involving domino tableaux, Electron. J. Combin. 7 (2000), no. 1, R35.

[Zel81] Zelevinsky, A. V., Representations of Finite Classical Groups: A Hopf Algebra Approach, Lecture Notes in Mathematics, no. 869, Springer-Verlag, 1981.

Department of Mathematics, Massachusetts Institute of Technology, Cambridge, MA 02139-4307, U.S.A.

E-mail address: sylvaner@math.mit.edu 